\title{
FOURIER INTEGRAL OPERATORS. II
}

BY

\author{
J. J. DUISTERMAAT and L. HÖRMANDER
}

University of Nijmegen, Holland, and University of Lund, Sweden (1)

\section{Preface}

The purpose of this paper is to give applications of the operator theory developed in the first part (Acta Math., 127 (1971), 79-183). These concern the existence and regularity of solutions of a pseudo-differential equation

$$
P u=f
$$

in a manifold $X$. In particular we construct and study parametrices for $P$.

In the first chapter, Chapter $\mathrm{V}$, we have collected some general facts concerning the calculus of Fourier integral operators needed later. In Chapter VI we then consider the equation ( 1 ) under the assumption that $P$ has a principal symbol $p$ which is homogeneous of degree $m$ and real. First we study the propagation of singularities of solutions of (1). If no bicharacteristic curve of $P$ is contained in a compact set in $X$ this leads to semi-global existence theorems and we can then give necessary and sufficient conditions for the operator $P$ to map $\mathscr{D}^{\prime}(X) / C^{\infty}(X)$ onto itself. Globally as well as locally these hypotheses are weaker than those made in Hörmander [17, Chap. VIII]. Under the same hypotheses we construct (twosided) parametrices for $P$, that is, inverses $\bmod C^{\infty}$. If the characteristic set is split in a disjoint union of open and closed subsets $N^{+}$and $N^{-}$there is $\bmod C^{\infty}$ a unique parametrix $E=E\left(N^{+}\right)$such that for $f \in \mathscr{E}^{\prime}(X)$ the wave front set of $E f$ in addition to that of $f$ only contains forward (backward) bicharacteristic half strips emanating from points in $N^{+}$(resp. $\left.N^{-}\right)$. When the characteristic set has $k$ components there are $2^{k}$ such parametrices. For the Klein-Gordon equation $\square+m^{2}$ these are given by the advanced and retarded fundamental solutions, the Feynman "propagator" and its complex conjugate. The difference between the Feynman propagator and the advanced or retarded fundamental

(1) Supported in part by NSF Grant GP-27176 at Courant Institute, New York University, NSF Grant GP-7952X2 at the Institute for Advanced Study, Princeton, and AFOSR contract F44620-69-C0106 at Stanford University. 
solution is $\pm \sqrt{-1}$ times the positive definite distribution which is the Fourier transform of the Lorentz invariant measure on either component of the hyperboloid $\xi_{1}^{2}-\ldots-\xi_{n}^{2}=m^{2}$. (See e.g. Bjorken and Drell [32].) On physical grounds it has been suggested (see DeWitt [34]) that this positivity remains valid for the wave operator on a pseudo-Riemannian manifold of Lorentz signature. In this direction we prove here that $E\left(N^{+}\right) / i$ increases with $N^{+}$(in the sense of operators in Hilbert space) if $P$ is self-adjoint and the indetermined $C^{\infty}$ part of $E$ is conveniently chosen.

In Chapter VII we drop the hypothesis that the principal part $p$ of $P$ is real, but we require that the differentials of $\operatorname{Re} p$ and $\operatorname{Im} p$ are linearly independent and that the Poisson bracket $\{\operatorname{Re} p, \operatorname{Im} p\}$ vanishes at the zeros of $p$. Even under this strong restriction our results are less complete than in the real case. The more general classes of operators studied recently by Nirenberg-Trèves [25] and Egorov [8] are not considered here at all. Nor do we discuss operators between sections of vector bundles although this involves no additional difficulty when the fiber dimensions are the same and hypotheses are made on the determinant as in the scalar case. For a more detailed description of the contents we refer to the introductions of each chapter.

The authors would like to thank Professor A. Wightman for patient instruction on the role of "propagators" in relativistic quantum mechanics. This led to the results of section 6.6 indicated above.

\section{Additional results on the calculus}

\subsection{Introduction}

In section 5.1 we shall just recall with slight extensions some of the notions introduced in part I. The following two sections give an important supplement to the multiplicative properties of Fourier integral operators proved in section 4.2. There we proved that if $A_{1} \in I_{Q}^{m_{1}}\left(X \times Y, C_{1}^{\prime}\right), A_{2} \in I_{Q}^{m_{2}}\left(Y \times Z, C_{2}^{\prime}\right)$, if the composition $C_{1} \circ C_{2}$ of the canonical relations $C_{1}$ and $C_{2}$ is well defined and $A_{1}$ or $A_{2}$ is properly supported, then $A_{1} A_{2} \in I_{Q}^{m_{1}+m_{2}}(X \times Z$, $\left.\left(C_{1} \circ C_{2}\right)^{\prime}\right)$, and we computed the principal symbol. However, for some choices of $A_{1}$ and $A_{2}$ which occur in Chapters VI and VII the principal symbol of the product will vanish identically although this is not the case for either factor. Thus $A_{1} A_{2} \in I_{Q}^{m_{1}+m_{3}+1-2 o}\left(X \times Z,\left(C_{1} \circ C_{2}\right)^{\prime}\right)$ then, and we must compute the principal symbol of order $m_{1}+m_{2}+1-2 \varrho$. In doing so we will assume for the sake of simplicity that $A_{1}$ is a pseudo-differential operator of type 1,0 . Besides the principal symbol of $A_{1}$ we must also consider an invariantly defined subprincipal symbol closely related to the subcharacteristic of Gårding-Kotake-Leray [37]. This is discussed in section 5.2 and the required formula for the principal symbol of the product is given in 
section 5.3. It involves a first order differential operator depending on $A_{1}$ which acts on the principal symbol of $A_{2}$. The solvability of the equation $A_{1} A_{2}=B$ with given $A_{1}$ and $B$ is also discussed in section 5.3 under the assumption that the first order differential equation for the principal symbol of $A_{2}$ can be solved. This is actually the transport equation of geometrical optics. Instances of this will be encountered in Chapters VI and VII. In section 5.4 finally we examine when a space $I_{Q}^{m}(X, \Lambda)$ is included in the space $H_{(s)}(X)$ of distributions with locally square integrable derivatives of order $s$.

\subsection{Preliminaries}

Let $X$ be a $C^{\infty}$ manifold and $\Lambda$ a closed conic Lagrangean submanifold of $T^{*}(X) \backslash 0$ where $T^{*}(X)$ is the cotangent bundle and 0 the zero section. (We require manifolds to be countable at infinity.) In section 3.2 we have introduced spaces $I_{\varrho}^{m}(X, \Lambda)\left(m \in \mathbf{R}, \frac{1}{2}<\varrho \leqslant 1\right)$ of distribution densities of order $\frac{1}{2}$ in $X$. If $A \in I_{\varrho}^{m}(X, \Lambda)$ then the wave front set $W F(\mathrm{~A})$ is contained in $\Lambda$ and there is a natural isomorphism

$$
I_{\varrho}^{m}(X, \Lambda) / I_{\varrho}^{m+1-2 \varrho}(X, \Lambda) \cong S_{\varrho}^{m+n / 4}\left(\Lambda, \Omega_{\frac{1}{2}} \otimes L\right) / S_{\varrho}^{m+n / 4+1-2 \varrho}\left(\Lambda, \Omega_{\frac{1}{2}} \otimes L\right)
$$

If $a \in S_{Q}^{m+n / 4}\left(\Lambda, \Omega_{\frac{1}{2}} \otimes L\right)$ and $u \in I_{e}^{m}(X, \Lambda)$ have corresponding residue classes we call $a$ a principal symbol of $u$. The following result is really implicit in Chapters I and III but we state it for later reference:

Proposition 5.1.1. Let $A_{k} \in I_{\varrho}^{m_{k}}(X, \Lambda), k=0,1,2, \ldots$ where $m_{k} \rightarrow-\infty$ as $k \rightarrow \infty$. Set $m_{k}^{\prime}=\max _{j \geqslant k} m_{j}$. Then one can find $A \in I_{\mathrm{e}}^{m_{\mathrm{o}}^{\prime}}(X, \Lambda)$ such that

$$
A-\sum_{j<k} A_{j} \in I_{\varrho}^{m_{k}^{\prime}}(X, \Lambda), \quad k=1,2, \ldots
$$

$A$ is uniquely determined modulo $C^{\infty}(X)$ and has the same property relative to any rearrange. ment of the series $\Sigma A_{j}$; we write $A \sim \Sigma A_{j}$.

Here we have written $C^{\infty}(X)$ for the space of $C^{\infty}$ densities of order $\frac{1}{2}$. Similarly we shall often write just $\mathscr{D}^{\prime}(X)$ for the distribution densities of order $\frac{1}{2}$.

Proof. If every $A_{k}$ is of the form (3.2.14) with a fixed $j$ and an amplitude of order $m_{k}+\left(n-2 N_{j}\right) / 4$ satisfying the hypotheses of Definition 3.2 .2 with a fixed $j$ and $K$, then the proposition is an immediate consequence of Proposition 1.1.9. We can choose a countable subset $J_{0}$ of the index set $J$ used there such that the sets $X_{j}^{\prime}$ are a locally finite covering of 
$X$ and $U$, are a locally finite covering of $\Lambda$. Each $A_{k}$ can then by Theorem 3.2.I be split into a sum $\Sigma A_{k j}$ with $A_{k j}$ as above. Thus $\Sigma_{k} A_{k j}$ is defined modulo $C^{\infty}$ and since the supports of these distributions are locally finite we can form the sum $A$ with respect to $j$ also. It is obvious that it has the desired property.

Extending the definition of characteristic used in section 2.5 for pseudo-differential operators we shall say that $A \in I_{g}^{m}(X, \Lambda)$ is non-characteristic at $\lambda \in \Lambda$ if and only if any principal symbol $a \in S_{e}^{m+n / 4}\left(\Lambda, \Omega_{\frac{1}{2}} \otimes L\right)$ has a reciprocal $b \in S_{Q}^{-m-n / 4}\left(\Lambda, \Omega_{-\frac{1}{2}} \otimes L^{-1}\right)$ in a conic neighborhood of $\lambda$. If $s_{0}$ is a local section of $\Omega_{i} \otimes L$ which has no zero and is homogeneous of order $m+n / 4$, this means precisely that $a=a_{0} s_{0}$ where $a_{0}$ is complex valued and $\left|a_{0}\right|$ is bounded from below at infinity in a conic neighborhood of $\lambda$. This condition is of course independent of the choice of principal symbol. By Theorem 3.2.6 the non-characteristic points belong to $W F(A)$.

If $X$ and $Y$ are two $C^{\infty}$ manifolds then a homogeneous canonical relation from $T^{*}(Y) \backslash 0$ to $T^{*}(X) \backslash 0$ was defined in section 4.1 to be a conic $C^{\infty}$ submanifold $C$ of $\left(T^{*}(X) \backslash 0\right) \times\left(T^{*}(Y) \backslash 0\right)$ which is Lagrangean with respect to the difference $\sigma_{X}-\sigma_{Y}$ of the symplectic forms and is closed in $T^{*}(X \times Y) \backslash 0$. Then the manifold $C^{\prime}$ obtained by multiplication with -1 in the fibers of $T^{*}(Y)$ is Lagrangean in $T^{*}(X \times Y) \backslash 0$, and the elements of $I_{Q}^{m}\left(X \times Y, C^{\prime}\right)$ can be considered as maps from $\mathscr{E}^{\prime}(Y)$ to $\mathscr{D}^{\prime}(X)$ and $C_{0}^{\infty}(Y)$ to $C^{\infty}(X)$.

The following is an immediate extension of Proposition 2.5.1:

Proposition 5.1.2. Let $C$ be a bijective homogeneous canonical transformation from $T^{*}(Y) \backslash 0$ onto $T^{*}(X) \backslash 0$, thus $\operatorname{dim} X=\operatorname{dim} Y$, and assume that $A \in I_{e}^{m}\left(X \times Y, C^{\prime}\right)$ is properly supported and elliptic, that is, has no characteristic points. Then there exists a properly supported elliptic Fourier integral operator $B \in I_{Q}^{-m}\left(Y \times X,\left(C^{-1}\right)^{\prime}\right)$ which is a left and right parametrix, that is, $B A-I$ and $A B-I$ have $C^{\infty}$ kernels. Any other parametrix for $A$ differs from $B$ by an operator with $C^{\infty}$ kernel.

Proof. We can regard the principal symbol $a$ of $A$ as an element of $S_{e}^{m}\left(C, L_{C}\right)$ (see 4.1.7). The ellipticity means that we can find $b \in S_{\varrho}^{-m}\left(C, L_{C}^{-1}\right)$ such that over any compact set $b a=1$ outside a large enough sphere. Choose $B_{0} \in I_{e}^{-m}\left(Y \times X,\left(C^{-1}\right)^{\prime}\right)$ with this principal part. Then we obtain that $A B_{0}=I+R_{1}, B_{0} A=I+R_{2}$ where $R_{j}$ are pseudo-differential operators in $X$ and $Y$ respectively of degree 1-2 $\varrho<0$ and type $\varrho$. As in the proof of Proposition 2.5.I it follows that $I+R_{j}$ has a twosided parametrix which is $\sim \Sigma_{0}^{\infty}\left(-R_{j}\right)^{k}$. It follows that $A$ has both left and right parametrices and since the proof of Proposition 2.5.1 gives without change that left and right parametrices must differ by operators with $C^{\infty}$ kernel, the proposition is proved. 
We shall sometimes need local versions of the preceding notions and results. Thus let $\Lambda$ be a conic Lagrangean manifold in $T^{*}(X) \backslash 0$ which is not necessarily closed, and let $K$ be a conic subset which is closed in $T^{*}(X) \backslash 0$. We then denote by $I_{\varrho}^{m}(X, K)$ the set of distributions introduced in Definition 3.2.2 with the additional condition that for each $j \in J$ the restriction of $a_{j}$ to some conic neighborhood in $\mathbf{R}^{n} \times \mathbf{R}^{N_{j}}$ of the pullback of $\Lambda \backslash K$ by the mapping $C_{\varphi_{i}} \ni(x, \theta) \rightarrow\left(x, \varphi_{j x}^{\prime}\right)$ is of class $S^{-\infty}$. Note that the definition depends both on $K$ and on $\Lambda$ although $\Lambda$ has been suppressed in the notation. The proof of Theorem 3.2.5 again gives an isomorphism

$$
S_{\varrho}^{m+n / 4}\left(K, \Omega_{\frac{1}{2}} \otimes L\right) / S_{\varrho}^{m+n / 4+1-2 \varrho}\left(K, \Omega_{\frac{1}{2}} \otimes L\right) \cong I_{\varrho}^{m}(X, K) / I_{\varrho}^{m+1-2 \varrho}(X, K)
$$

where $S_{\varrho}^{\mu}\left(K, \Omega_{\frac{1}{2}} \otimes L\right)$ denotes the set of $a \in S_{\varrho}^{\mu}\left(\Lambda, \Omega_{\frac{1}{2}} \otimes L\right)$ such that $a \in S^{-\infty}$ on $\Lambda \backslash K$. Clearly the analogues of Theorem 3.2.6 and Proposition 5.1.1 are also valid.

Now let $C$ be the graph of a homogeneous canonical transformation from a conic neighborhood of $\left(y_{0}, \eta_{0}\right) \in T^{*}(Y) \backslash 0$ to a conic neighborhood of $\left(x_{0}, \xi_{0}\right) \in T^{*}(X) \backslash 0$ with $c_{0}=\left(\left(x_{0}, \xi_{0}\right),\left(y_{0}, \eta_{0}\right)\right) \in C$. Let $A \in I_{Q}^{m}\left(X \times Y, K^{\prime}\right)$ where $K$ is a conic subset of $C$ which is closed in $T^{*}(X \times Y) \backslash 0$, and assume that $c_{0}$ is a non-characteristic point for $A$. (We consider the principal symbol to be defined on $C$ or on $C^{\prime}$, whichever is more convenient for the moment. This should cause no confusion.) Then the proof of Proposition 5.1.2 shows that one can find $B \in I_{\varrho}^{-m}\left(Y \times X, K_{1}^{\prime}\right)$ where $K_{1}$ is the inverse of $K$, such that

$$
\left(x_{0}, \xi_{0}\right) \notin W F\left(A B-I_{X}\right), \quad\left(y_{0}, \eta_{0}\right) \notin W F\left(B A-I_{Y}\right) .
$$

Here $I_{X}$ and $I_{Y}$ are the identity operators in $\mathscr{D}^{\prime}(X)$ and $\mathscr{D}^{\prime}(Y)$ respectively. Note that $A B$ and $B A$ are pseudo-differential operators.

\subsection{The subprincipal symbol of a pseudo-differential operator}

Let $X$ be a $C^{\infty}$ manifold and $P \in L_{e}^{m}(X)$ a pseudo-differential operator in $X$ regarded as a map from densities of order $\frac{1}{2}$ to densities of order $\frac{1}{2}$. For every choice of local coordinates $x_{1}, \ldots, x_{n}$ in an open subset $X_{1}$ the operator has a symbol $p(x, \xi),(x, \xi) \in X_{1} \times \mathbf{R}^{n}$, determined modulo $S^{-\infty}$ (see section 2.1). Regarded as a function on $T^{*}(X)$ it is independent of the choice of local coordinates modulo $S_{\varrho}^{m+1-2 e}$. We shall improve this invariance by a modification of an argument of Gårding-Kotake-Leray [37] which takes advantage of the densities of order $\frac{1}{2}$.

Let $\varphi_{1}, \ldots, \varphi_{n}$ be another system of local coordinates in $X_{1}$ and set $\varphi(x, \theta)=\Sigma \varphi_{j}(x) \theta_{j}$, $(x, \theta) \in X_{1} \times \mathbf{R}^{n}$. Choosing a density $w \in C_{0}^{\infty}\left(X_{1}\right)$ of order $\frac{1}{2}$ we shall consider $e^{-i \varphi} P\left(w e^{i \varphi}\right)$ as a function on $T^{*}\left(X_{1}\right)$ by means of the vector bundle isomorphism $(x, \theta) \rightarrow\left(x, \varphi_{x}^{\prime}(x, \theta)\right)$. This quantity may of course depend on $\varphi_{1}, \ldots, \varphi_{n}$ but by evaluating it in terms of the local 
coordinates $x_{1}, \ldots, x_{n}$ we shall see that this is not the case modulo $S_{\varrho}^{m+2(1-2 \varrho)}$ and so we will obtain the desired invariance.

In terms of the local coordinates we have

$$
e^{-i \varphi} P\left(w e^{i \varphi}\right) \sim \sum p^{(\alpha)}\left(x, \varphi_{x}^{\prime}\right) D_{z}^{\alpha}\left(w(z) e^{i \varrho(x, z, \theta)}\right) /\left.\alpha !\right|_{z=x}
$$

where

$$
\varrho(x, z, \theta)=\varphi(z, \theta)-\varphi(x, \theta)-\left\langle z-x, \varphi_{x}^{\prime}(x, \theta)\right\rangle \text {. }
$$

This follows from the formulas for multiplication of pseudo-differential operators and for changing variables given in section 2.1, and can also be taken as a starting point for a proof of these (see Hörmander [13, section 2]). If $k$ is the largest integer $\leqslant|\alpha| / 2$, the second factor in (5.2.1) is a polynomial in $\theta$ of degree $\leqslant k$ and therefore in $S_{1}^{k}$. This gives easily

$$
\begin{aligned}
e^{-i \varphi} P\left(w e^{i \varphi}\right)=p & \left(x, \varphi_{x}^{\prime}\right) w(x)+\sum p^{(j)}\left(x, \varphi_{x}^{\prime}\right) D_{j} w(x) \\
& +(2 i)^{-1} \sum p^{(j k)}\left(x, \varphi_{x}^{\prime}\right) w(x) \partial^{2} \varphi / \partial x ; \partial x_{k} \quad \bmod S_{e}^{m+2(1-2 \varrho)}
\end{aligned}
$$

Note that only the first term on the right hand side is not determined by the residue class of $p \bmod S_{Q}^{m+1-2 \varrho}$. Since we should regard $w$ as a density of order $\frac{1}{2}$ it is natural to replace the first sum by a Lie derivative so we digress to recall this notion.

If $v$ is a real $C^{\infty}$ vector field and $a$ a density of order $\varkappa$ on a manifold $M$, then the Lie derivative of $a$ with respect to $v$ is defined by

$$
\mathscr{L}_{v} a=\left.\frac{d}{d t}\left(\varphi^{t}\right)^{*} a\right|_{t=0}
$$

Here $\varphi^{t}$ is the local one parameter group of transformations of $M$ generated by $v$ and $\varphi^{*} a$ denotes the pullback of $a$ by means of the transformation $\varphi$. If $a_{0}$ is a non-vanishing density and we write $a=u a_{0}$, then

$$
\mathscr{L}_{v} a=(v u) a_{0}+u \mathscr{L}_{v} a_{0}=(v u+f u) a_{0}
$$

where $f$ is the function defined by $\mathscr{L}_{v} a_{0}=f a_{0}$. Apart from lower order terms Lie derivation of densities therefore coincides with differentiation of the factor $u$ with respect to the vector field $v$. If $a_{0}$ is the density of order $\varkappa$ corresponding to the standard volume in some local coordinates $y_{1}, \ldots, y_{k}$ on $M$ then

$$
f=\varkappa \operatorname{div} v=\varkappa \Sigma \partial v_{j} / \partial y_{j} .
$$

Formulas (5.2.4) and (5.2.5) define $\mathscr{L}_{v}$ also for complex vector fields.

Let $v$ be the vector field $\left(p^{(1)}\left(x, \varphi_{x}^{\prime}\right), \ldots, p^{(n)}\left(x, \varphi_{x}^{\prime}\right)\right)$ in $X_{1}$ which is defined by the function $p(x, \xi)$ on $T^{*}(X)$, with no other reference to the local coordinates $x_{1}, \ldots, x_{n}$. Since 


$$
\sum p^{(j k)}\left(x, \varphi_{x}^{\prime}\right) \partial^{2} \varphi\left(\partial x_{j} \partial x_{k}-\sum \partial p^{(j)}\left(x, \varphi_{x}^{\prime}\right) / \partial x_{j}=-\sum p_{(j)}^{(j)}\left(x, \varphi_{x}^{\prime}\right)\right.
$$

where $p_{(k)}^{(j)}(x, \xi)=\partial^{2} p / \partial x_{k} \partial \xi_{j}$, we obtain

$$
e^{-i \varphi} P\left(w e^{i \varphi}\right)=\left(p\left(x, \varphi_{x}^{\prime}\right)-(2 i)^{-1} \sum p_{(j)}^{(j)}\left(x, \varphi_{x}^{\prime}\right)\right) w(x)-i \mathscr{L}_{v} w \quad \text { modulo } S_{\ell}^{m+2(1-2 e)} .
$$

But $\mathscr{L}_{v} w$ is modulo $S_{e}^{m+2(1-2 e)}$ independent of the choice of local coordinates $x_{1}, \ldots, x_{n}$, so it follows that

$$
p\left(x, \varphi_{x}^{\prime}\right)-(2 i)^{-1} \sum p_{(j)}^{(j)}\left(x, \varphi_{x}^{\prime}\right)
$$

is also independent of the local coordinates $\bmod S_{e}^{m+2(1-2 e)}$ when regarded as a function on $T^{*}\left(X_{1}\right)$. Thus we have proved

PROPOSITION 5.2.1. Let $P$ be a pseudo-differential operator in a manifold $X$, considered as an operator between densities of order $\frac{1}{2}$. If $P \in L_{\dot{e}}^{m}$ and for some choice of local coordinates $p(x, \xi)$ denotes the full symbol, then

$$
p-(2 i)^{-1} \sum \partial^{2} p / \partial x_{j} \partial \xi_{j} \in S_{e}^{m}
$$

is modulo $S_{Q}^{m+2(1-2 g)}$ independent of the choice of local coordinates.

It is of course also possible to prove Proposition 5.2.1 directly using the formulas of section 2.1 for changing variables but this approach would not explain the reason for the invariance as clearly.

In what follows we shall usually consider pseudo-differential operators $P \in L_{1}^{m}(X)$ with a homogeneous principal symbol $p$. This means that we assume the existence of a $C^{\infty}$ homogeneous function $p$ of degree $m$ on $T^{*}(X) \backslash 0$ such that for any system of local coordinates the full symbol of $P$ is of the form $p+r$ where $r \in S_{1}^{m-1}$. Clearly $p$ is then uniquely determined by $P$, and Proposition 5.2.1 reduces to the statement that

$$
r-(2 i)^{-1} \sum \partial^{2} p / \partial x_{j} \partial \xi_{j} \in S_{1}^{m-1}
$$

is uniquely determined modulo $S_{1}^{m-2}$. We can therefore choose $c \in S_{1}^{m-1}\left(T^{*}(X)\right)$ which agrees with (5.2.8) modulo $S_{1}^{m-2}$ for any choice of local coordinates. We shall call such a symbol $c$ a subprincipal symbol. If the full symbol of $P$ is a sum of homogeneous terms we can of course choose $c$ homogeneous of degree $m-1$, and the definition of $c$ is then unique.

\subsection{Products with vanishing principal symbol}

Let $X$ and $Y$ be $C^{\infty}$ manifolds and $C$ a homogeneous canonical relation from $T^{*}(Y) \backslash 0$ to $T^{*}(X) \backslash 0$. If $P$ is a properly supported pseudo-differential operator in $L_{e}^{m}(X)$ and if $A \in I_{e}^{m^{\prime}}\left(X \times Y, C^{\prime}\right)$, we know from Theorem 4.2.2 that $P A \in I_{Q}^{m+m^{\prime}}\left(X \times Y, C^{\prime}\right)$, and Theorem 
4.2.3 allows us to compute a principal symbol of $P A$. This may vanish identically, however, and the following result gives the principal symbol of lower order in such a case.

THEOREM 5.3.1. Let $P$ be a properly supported pseudo-differential operator $\in L_{1}^{m}(X)$ with homogeneous principal symbol $p$, and denote by $c$ a subprincipal symbol of $P$. Assume that $C$ is a homogeneous canonical relation from $T^{*}(Y) \backslash 0$ to $T^{*}(X) \backslash 0$ such that $p$ vanishes on the projection of $C$ in $T^{*}(X) \backslash 0$. If $A \in I_{Q}^{m^{\prime}}\left(X \times Y, C^{\prime}\right)$ and $a \in S^{m^{\prime}+\left(n_{X^{+}}+n_{\bar{Y}}^{\prime}\right) / 4}\left(C^{\prime}, \Omega_{\mathrm{f}} \otimes L\right)$ is a principal symbol of $A$, it follows that the product $P A \in I_{\mathrm{e}}^{m+m^{\prime}-\varrho}\left(X \times Y, C^{\prime}\right)$ has

$$
i^{-1} \mathscr{L}_{H_{p}} a+c a
$$

as principal symbol. Here $H_{p}$ is the Hamilton field of $p$ lifted to a function on $\left(T^{*}(X) \backslash 0\right) \times$ $\left(T^{*}(Y) \backslash 0\right)$, so $H_{p}$ is tangential to $C$, and $\mathscr{L}_{H_{p}}$ is the corresponding Lie derivative.

Note that since $L$ is flat we can define $\mathscr{L}_{H_{p}}$ a by using local trivializations of $L$ which only differ by a constant factor and therefore do not affect the definition.

Proof. It follows from Theorem 3.2.3 and an observation made at the end of its proof that a conic neighborhood of any point in $C^{\prime}$ can be represented by a phase function of the form

$$
\varphi(x, y, \xi, \eta)=\langle x, \xi\rangle+\langle y, \eta\rangle-H(\xi, \eta)
$$

provided that the local coordinates in $X$ and in $Y$ are conveniently chosen. Here $H$ is defined in a conic neighborhood of $\left(\xi_{0}, \eta_{0}\right) \in\left(\mathbf{R}^{n_{X}} \backslash 0\right) \times\left(\mathbf{R}^{n_{T}} \backslash 0\right)$. We may therefore assume in the proof that $A$ is of the form

$$
A u(x)=\iiint e^{i \varphi(x, y, \xi, \eta)} a(x, y, \xi, \eta) u(y) d y d \xi d \eta, \quad u \in C_{0}^{\infty},
$$

where $a \in S_{Q}^{\mu}$ for $\mu=m^{\prime}-\left(n_{X}+n_{Y}\right) / 4$ and $a$ vanishes outside a small conic neighborhood of $\left(H_{\xi}^{\prime}, H_{\eta}^{\prime}, \xi, \eta\right)$ with $\xi=\xi_{0}, \eta=\eta_{0}$. The map

$$
(\xi, \eta) \rightarrow\left(H_{\xi}^{\prime}, H_{\eta}^{\prime}, \xi, \eta\right)
$$

is a local parametrization of the manifold $C_{\varphi}$ of points where $\varphi$ is critical with respect to $(\xi, \eta)$, and the density defined in $C_{\varphi}$ by the pullback of the Dirac measure in $\mathbf{R}^{n_{X}+n_{Y}}$ with the map $(x, y, \xi, \eta) \rightarrow\left(x-H_{\xi}^{\prime}, y-H_{\eta}^{\prime}\right)$ coincides with the Lebesgue measure in $(\xi, \eta)$. In terms of these local parameters on $C^{\prime}$ and the trivialization of $L$ given by the phase function $\varphi$, a principal symbol of $A$ is therefore given by the restriction $a\left(H_{\xi}^{\prime}, H_{\eta}^{\prime}, \xi, \eta\right)$.

By repeated use of Proposition 1.2.5 we can replace $a$ by a function $a_{0}(\xi, \eta)$ without changing the singularities of $A$ in the local coordinate patch considered in (5.3.2). An application of $P$ under the sign of integration now gives 


$$
P A u(x)=\iiint e^{i \varphi(x, y, \xi, \eta)}(p(x, \xi)+r(x, \xi)) a_{0}(\xi, \eta) u(y) d y d \xi d \eta
$$

where as at the end of section 5.2 we have denoted the full symbol of $P$ by $p+r$. Since $p\left(H_{\xi}^{\prime}, \xi\right)=\mathbf{0}$ we can choose by means of Taylor's formula functions $p_{j} \in C^{\infty}$ in a neighborhood of the support of the integrand in (5.3.3) such that

$$
p(x, \xi)=\sum p_{j}(x, \xi, \eta)\left(x_{j}-\partial H / \partial \xi_{j}\right)=\sum p_{j}(x, \xi, \eta) \partial \varphi(x, y, \xi, \eta) / \partial \xi_{j}
$$

and $p_{j}$ is homogeneous of degree $m$ with respect to $(\xi, \eta)$. We may assume that $a_{0}$ vanishes in a neighborhood of 0 and obtain by an integration by parts with respect to $\xi$ as in section 1.2

$$
P A u(x)=\iiint e^{i \eta(x, y . \xi . \eta)}\left(r a_{0}-i^{-1} \sum \partial\left(p_{j}(x, \xi, \eta) a_{0}(\xi, \eta)\right) / \partial \xi_{j}\right) u(y) d y d \xi d \eta
$$

$P A$ is therefore a Fourier integral operator with the amplitude

$$
r a_{0}-i^{-1} \sum p_{j}(x, \xi, \eta) \partial a_{0}(\xi, \eta) / \partial \xi_{j}-i^{-1} a_{0}(\xi, \eta) \Sigma \partial p_{j}(x, \xi, \eta) / \partial \xi_{j}
$$

This is in $S_{e}^{m+\mu-\varrho}$ so it follows that $P A \in I_{\varrho}^{m+m^{\prime}-\varrho}$. We can obtain the principal symbol by taking the restriction of (5.3.6) to $C_{\varphi}$. In doing so we may replace $a_{0}(\xi, \eta)$ by $a\left(H_{\xi}^{\prime}, H_{\eta}^{\prime}\right.$, $\xi, \eta)$ for the difference is in $S_{\varrho}^{\mu+1-2 \varrho}$. With $x=H_{\xi}^{\prime}, y=H_{\eta}^{\prime}$ this gives

$$
-\sum p_{j}(x, \xi, \eta) \partial a\left(H_{\xi}^{\prime}, H_{\eta}^{\prime}, \xi, \eta\right) / \partial \xi_{j}=-\sum p_{j}\left(\sum \partial a / \partial x_{k} \partial^{2} H / \partial \xi_{k} \partial \xi_{j}+\sum \partial a / \partial y_{k} \partial^{2} H / \partial \eta_{k} \partial \xi_{j}+\partial a / \partial \xi_{j}\right)
$$

Since differentiation of (5.3.4) gives for $x=H_{\xi}^{\prime}$

$$
\partial p / \partial x_{j}=p_{j}, \quad \partial p / \partial \xi_{k}=-\sum p_{j} \partial^{2} H / \partial \xi_{j} \partial \xi_{k}, \quad 0=-\sum p_{j} \partial^{2} H / \partial \xi_{j} \partial \eta_{k}
$$

we conclude that

$$
-\sum p_{j}(x, \xi, \eta) \partial a\left(H_{\xi}^{\prime}, H_{\eta}^{\prime}, \xi, \eta\right) / \partial \xi_{j}=\Sigma\left(\partial p / \partial \xi_{j} \partial a / \partial x_{j}-\partial p / \partial x_{j} \partial a / \partial \xi_{j}\right)
$$

This shows that the vector field $-\Sigma p_{j} \partial / \partial \xi_{j}$ on $C_{\varphi}$ is the expression of $H_{p}$ in terms of the local coordinates $(\xi, \eta)$ there. Therefore the Lie derivative along $H_{p}$ of $a\left(H_{\xi}^{\prime}, H_{\eta}^{\prime}, \xi, \eta\right)$ considered as a density of order $\frac{1}{2}$ is in view of (5.2.5) equal to

$$
-\sum p_{j} \partial a_{0} / \partial \xi_{j}-\frac{1}{2} \sum \partial p_{j}\left(H_{\xi}^{\prime}, \xi, \eta\right) / \partial \xi_{j} a_{0}
$$

Thus the principal symbol of $P A$ is $i^{-1} \mathscr{L}_{H_{p}} a+\gamma a$ where if we recall (5.3.6)

$$
\gamma=r+(2 i)^{-1} \sum \partial p_{j}\left(H_{\xi}^{\prime}, \xi, \eta\right) / \partial \xi_{j}-i^{-1} \sum \partial p_{j}(x, \xi, \eta) / \partial \xi_{j}, \quad x=H_{\xi}^{\prime} .
$$

13-722909 Acta mathematica 128. Imprimé le 23 Mars 1972. 
This is the value for $x=H_{\xi}^{\prime}$ of

$$
\begin{aligned}
r+(2 i)^{-1}\left(\sum \partial p_{j} / \partial \xi_{j}+\sum \partial p_{j} / \partial x_{k} \partial^{2} H / \partial \xi_{k} \partial \xi_{j}-2 \sum \partial p_{j} / \partial \xi_{j}\right) \\
\quad=r-(2 i)^{-1} \sum \partial / \partial \xi_{j}\left(p_{j}+\sum \partial p_{k} / \partial x_{j}\left(x_{k}-\partial H / \partial \xi_{k}\right)\right)=r-(2 i)^{-1} \sum \partial^{2} p / \partial x_{j} \partial \xi_{j}
\end{aligned}
$$

where the last equality follows from another application of (5.3.4). This completes the proof, for the right hand side is the definition 5.2.8 of the subprincipal part.

From the proof it is obvious that there is also a local version of Theorem 5.3.1 when $A \in I_{\varrho}^{m^{\prime}}\left(X \times Y, K^{\prime}\right)$ for a closed conic subset $K$ of a canonical relation $C$.

Keeping the hypotheses of Theorem 5.3.1 we shall now discuss the solvability of the equation $P A=B$ with $A \in I_{e}^{m^{\prime}}\left(X \times Y, C^{\prime}\right)$ when $B$ is given. We know by Theorem 5.3.1 that $B$ must be in $I_{\ell}^{m+m^{\prime}-\varrho}\left(X \times Y, C^{\prime}\right)$ and that the principal symbol $b \in S_{Q}^{m+m^{\prime}-\varrho+n / 4}$ is given by

$$
b=i^{-1} \mathscr{L}_{H_{p}} a+c a .
$$

Here we have written $n=\operatorname{dim} X+\operatorname{dim} Y$. However, we cannot expect this equation to have a solution $a \in S_{\varrho}^{m^{\prime}+n / 4}$ for every $b \in S_{Q}^{m+m^{\prime}-\varrho+n / 4}$ if $\varrho<1$. For example, if $m=1$ we might have $H_{p}=\partial / \partial x_{1}$ and integration of $b$ with respect to $x_{1}$ would not decrease the order. On the other hand, this example indicates that it is reasonable to expect such a solution $a$ if $b \in S_{a}^{m^{\prime}+m-1+n / 4}$. In fact, such situations will be encountered in Chapters VI and VII, and the conclusions are discussed in the following analogue of Proposition 5.1.2.

TH о о Eм 5.3.2. Let $P$ and $C$ satisfy the hypotheses in Theorem 5.3.1, let $\varrho>2 / 3$ and assume that for every real $\mu$

$$
H_{\mathfrak{p}} S_{\mathrm{e}}^{\mu}(C) \supset S_{e}^{m-1+\mu}(C)
$$

For every $B \in I_{e}^{m+m^{\prime}-1}\left(X \times Y, C^{\prime}\right)$ one can then find $A \in I_{Q}^{m^{\prime}}\left(X \times Y, C^{\prime}\right)$ such that $P A-B$ has a $C^{\infty}$ kernel. If $b$ is the principal symbol of $B$ and $a$ is any solution of (5.3.9) belonging to $S_{e}^{m^{\prime}+n / 4}\left(C, L \otimes \Omega_{\frac{1}{2}}\right)$ one can choose $A$ with principal symbol in the class of a modulo $S_{e}^{m^{\prime}+n / 4+2-3 e}$ $\left(C, L \otimes \Omega_{\frac{1}{2}}\right)$.

Proof. From (5.3.10) it follows that (5.3.9) has a solution $a \in S_{e}^{\mu}\left(C^{\prime}, L \otimes \Omega_{1}\right)$ for any $b \in S_{Q}^{m+\mu-1}\left(C^{\prime}, L \otimes \Omega_{\frac{1}{2}}\right)$. In fact, let $\omega$ be a non-vanishing section of $L \otimes \Omega_{\frac{1}{4}}$ which is homogeneous of degree 0 . That such sections exist follows from the triviality of $L$ and $\Omega_{13}$ as complex vector bundles (see section 3.2 , p. 148). Writing $a=a_{0} \omega$ and $b=b_{0} \omega$ we reduce the equation (5.3.9) to the form

$$
\left(-i H_{p} a_{0}+c^{\prime} a_{0}\right)=b_{0}
$$

where $c^{\prime} \in S_{1}^{m-1}$ and $a_{0}, b_{0}$ denote sealar symbols of the same orders as $a, b$. By hypothesis 
we can find $\gamma \in S_{\ell}^{0}$ so that $H_{p} \gamma=c^{\prime}$. By Proposition 1.1 .8 we have exp $( \pm i \gamma) \in S_{\ell}^{0}$, and if we write $a_{0}=i e^{-i \gamma} a_{1}, b_{0}=e^{-i \gamma} b_{1}$ the equation reduces to

$$
H_{p} a_{1}=b_{1}
$$

which by hypothesis has a solution $a_{1} \in S_{Q}^{\mu}$ if $b_{1} \in S_{Q}^{m+\mu-1}$.

Now choose $A_{0} \in I_{e}^{m^{\prime}}\left(X \times Y, C^{\prime}\right)$ with principal symbol satisfying (5.3.9), and set

$$
B_{1}=B-P A_{0} .
$$

By Theorem 5.3.1 we obtain $B_{1} \in I_{Q}^{\mu}$ where $\mu=m+m^{\prime}-\varrho-(2 \varrho-1)=m+m^{\prime}-1-(3 \varrho-2)$. Iterating this argument we obtain sequences $A_{j} \in I_{e}^{m^{\prime}-j(3 e-2)}\left(X \times Y, C^{\prime}\right)$ and $B_{j} \in$ $I_{\ell}^{m+m^{\prime}-1-j(3 Q-2)}\left(X \times Y, C^{\prime}\right)$ such that $B_{0}=B$ and

$$
B_{j+1}=B_{j}-P A_{j}, \quad j=0,1,2, \ldots
$$

Let $A$ be the asymptotic sum of all $A_{j}, j=0,1, \ldots$ which is defined according to Proposition 5.1.1 since $3 \varrho>2$. Adding the equations (5.3.11) we obtain

$$
P\left(A_{0}+\ldots+A_{j}\right)=B_{0}-B_{j+1}
$$

which gives that $P A-B_{0} \in I_{e}^{-\infty}\left(X \times Y, C^{\prime}\right)=C^{\infty}(X \times Y)$. The theorem is proved.

Remark 1. A similar result is valid for the equation $A P=B$ for this is equivalent to the equation $P^{*} A^{*}=B^{*}$ for the adjoints.

Remark 2. We shall also need a local version of Theorem 5.3.2 where $C$ is replaced by a closed conic subset $K$ of a canonical relation which is not necessarily closed. Since the modifications involved are quite obvious we do not state this result explicitly.

\subsection{The smoothness of elements in $I_{o}^{m}(X, \Lambda)$}

First recall that $H_{(s)}(X)$ denotes the set of all $u \in \mathscr{Q}^{\prime}(X)$ such that $A u \in L_{100}^{2}(X)$ for all properly supported $A \in L_{1}^{s}(X)$. It is enough to require this for one elliptic $A$, and we have $B u \in H_{(s-m)}(X)$ if $u \in H_{(s)}(X)$ and $B \in L_{\varrho}^{m}(X), \varrho>\frac{1}{2}$. (See section 2.2 and also [13], section 5.) Let $\Lambda$ be a closed Lagrangean submanifold of $T^{*}(X) \backslash 0$.

THEOREM 5.4.1. $I_{Q}^{m}(X, \Lambda) \subset H_{(s)}(X)$ if and only if $m+n / 4+s<0$. Moreover, if $u \in$ $I_{o}^{m}(X, \Lambda)$ and $u$ has some non-characteristic point, then it follows that $u \notin H_{(s)}(X)$ when $0 \leqslant$ $m+n / 4+s$.

COROLLARY 5.4.2. A Fourier integral operator from $\mathscr{D}^{\prime}(Y)$ to $\mathscr{D}^{\prime}(X)$ is a HilbertSchmidt integral operator if it is of order $<-(\operatorname{dim} X+\operatorname{dim} Y) / 4$ and the kernel has compact support. 
It is interesting to compare this conclusion with Theorems 4.1.1 and 4.3.2

Proof of Theorem 5.4.1. It is sufficient to show that if $u \in I_{e}^{m}(X, \Lambda)$ and $W F(u)$ is in a small conic neighborhood $\Gamma$ of $\left(x_{0}, \xi_{0}\right) \in \Lambda$, then $u \in H_{(0)}=L_{\text {loc }}^{2}$ if $m+n / 4<0$ whereas $u \notin H_{(0)}$ if $m+n \backslash 4 \geqslant 0$ and $\left(x_{0}, \xi_{0}\right)$ is a non-characteristic point. Let $\chi$ be a homogeneous canonical transformation from a conic neighborhood of $\left(x_{0}, \xi_{0}\right)$ to a conic neighborhood of $\left(X_{0}, \Xi_{0}\right) \epsilon$ $T^{*}\left(\mathbf{R}^{n}\right) \backslash 0$ and let $K$ be a closed conic neighborhood of $\left(x_{0}, \xi_{0}, X_{0}, \Xi_{0}\right)$ in the graph of $\chi$. The inverse graph we denote by $K_{1}$. According to (5.1.2) we can choose operators $A \in I_{1}^{0}\left(X \times \mathbf{R}^{n}, K^{\prime}\right)$ and $B \in I_{1}^{0}\left(\mathbf{R}^{n} \times X,\left(K^{-1}\right)^{\prime}\right)$ such that

$$
\left(x_{0}, \xi_{0}\right) \notin W F(A B-I) .
$$

We may assume that $\Gamma$ does not meet $W F(A B-I)$, and then we have $A B u-u \in C^{\infty}$. Hence it follows from Theorem 4.3.1 that

$$
u \in L_{\mathrm{loc}}^{2} \Rightarrow B u \in L_{\mathrm{loc}}^{2} \Rightarrow A B u \in L_{\mathrm{loc}}^{2} \Rightarrow u \in L_{\mathrm{loc}}^{2}
$$

so $u \in L_{\text {loc }}^{2} \Leftrightarrow B u \in L_{\text {loc }}^{2}$. Now $B u \in I_{\varrho}^{m}\left(\mathbf{R}^{n}, \chi \Gamma\right)$, and by appropriate choice of $\chi$ we can make $\chi \Gamma$ contained in $0 \times\left(\mathbf{R}^{n} \backslash 0\right)$, the normal bundle of 0 in $\mathbf{R}^{n}$. In fact, with suitable local coordinates Theorem 3.1.3 shows that $\Lambda$ is defined near $\left(x_{0}, \xi_{0}\right)$ by $x=H^{\prime}(\xi)$ where $H$ is homogeneous of degree 1 near $\xi_{0}$, and we can take for $\chi$ the canonical transformation

$$
(x, \xi) \rightarrow\left(x-H^{\prime}(\xi), \xi\right) \text {. }
$$

In the special case to which the proof is now reduced we have

$$
u(x)=(2 \pi)^{-3 n / 4} \int e^{i\langle x, \theta\rangle} a(\theta) d \theta
$$

(the integral taken in the sense of Schwartz), where $a \in S_{\varrho}^{m-n / 4}$ and $1 / a \in S_{\varrho}^{-m+n / 4}$ in case $u$ has a non-characteristic point. By Parseval's formula we obviously have $u \in L_{\text {loc }}^{2}$ if $2(m-n / 4)<-n$, and not if $2(m-n / 4) \geqslant-n$ when there is a non-characteristic point, for $u$ is rapidly decreasing at infinity so $u \in L_{\text {loc }}^{2}$ if and only if $u \in L^{2}$.

\section{Pseudo-differential operators with real principal symbols}

\subsection{Introduction}

Throughout this chapter $P$ will denote a properly supported pseudo-differential operator of the class $L_{1}^{m}(X)$ where $X$ is a $C^{\infty}$ manifold, and we assume that $P$ has a real and homogeneous principal symbol $p$. In section 6.1 we show that if $P u=f \in C^{\infty}$ then the singularities of $u$ propagate along the bicharacteristies in the sense that $W F(u)$ is invariant under the 
flow defined by the Hamilton field of $p$. (Recall that by definition $p$ vanishes on $W F(u)$.) This implies that through every point in sing supp $u$ there is a bicharacteristic curve which stays in the singular support. (The conclusion is of course trivial if some such curve is reduced to a point.) In the constant coefficient case this result was first proved by Grušin [38]; a weaker statement valid also for variable coefficients had been given before in Hörmander [17, section 8.8]. A local extension of the Grušin theorem to the case of regular bicharacteristic curves was announced in Hörmander [15]. One of the main reasons for considering $W F(u)$ instead of sing supp $u$ is that this eliminates the difference between local and global results. For proofs using only pseudo-differential operators we refer to Hörmander [45], where only the case of a regular bicharacteristic curve is studied, and Hörmander [46] where an extension to certain operators with complex principal part is also given. Analogous results concerning non-analyticity have been given by Andersson [29], Kawai-Kashiwara (to be published) and Hörmander [44].

On the other hand, we prove in section 6.2 that there exist distributions $u$ with $P u \in C^{\infty}$ such that $W F(u)$ is generated by a given bicharacteristic strip. (The statement must be modified if the strip has boundary points. ) For earlier results in this direction see Zerner [53], Hörmander [17, section 8.8], [43]. The methods used in the proof are derived from the asymptotic expansions of geometrical optics but to obtain global results which do not require regularity of the bicharacteristic curve it is essential to use the calculus of Fourier integral operators.

In section 6.3 we use the results of sections 6.1 and 6.2 to study the existence of solutions of the equation $P u=f$. If no bicharacteristic curve of $P$ is contained in the compact set $K \subset X$ it is proved that the equation can be solved in a neighborhood of $K$ if and only if $f$ is orthogonal to the finite dimensional space of $v \in C_{0}^{\infty}(K)$ with ${ }^{t} P v=0$. If no bicharacteristic curve is contained in a compact subset of $X$ then the equation $P u=f$ has a solution modulo $C^{\infty}$ for every distribution $f$ if and only if $X$ is convex with respect to the bicharacteristic curves of $P$ in a sense explained in section 6.3. By an example it is shown that knowledge of the symbol alone cannot decide whether genuine solutions exist.

The existence theorems of section 6.3 are proved by abstract functional analysis but in section 6.5 we show that the same hypotheses on $P$ and $X$ allow one to construct parametrices of $P$, that is, inverses modulo $C^{\infty}$. As a preliminary for the construction we discuss existence theorems for real first order differential equations in section 6.4. This is required for the application of Theorem 5.3.2 and also gives an elementary example of the role of the convexity conditions occurring in the general case. In section 6.5 we determine in particular which sets that can carry $W F(E)$ when $E$ is a right, left or twosided parametrix. The results contain the theorems on the singularities of solutions of hyperbolic 
equations given by Hadamard [39] and Riesz [49] (second order equations) and CourantLax [33], Lax [21], Ludwig [22] (equations of arbitrary order). The study of parametrices is continued in section 6.6 which is mainly devoted to the positivity properties mentioned in the preface. These seem to be new even for second order hyperbolic operators with variable coefficients.

\subsection{The propagation of singularities}

The main result to be proved in this section is

THEOREM 6.1.1. Assume that $P \in L_{1}^{m}(X)$ is properly supported and has a real principal part $p$ which is homogeneous of degree $m$. If $u \in \mathscr{D}^{\prime}(X)$ and $P u=f$ it follows then that $W F(u) \backslash W F(f)$ is contained in $p^{-1}(0)$ and is invariant under the Hamiltonian vector field $H_{p}$.

We shall prove the theorem by reducing it to the special case $P=D_{n}$ in $\mathbf{R}^{n}$ where it follows by explicit solution of the equation $P u=f$. The study of this special case as well as the reduction will at the same time prepare for the construction of a parametrix in section 6.5, so we shall also include some material not required until later on.

By $E_{n}^{+}$and $E_{n}^{-}$we denote the forward and backward fundamental solutions of the operator $D_{n}$, the kernels of which are defined by

$$
E_{n}^{+}=i \delta\left(x^{\prime}-y^{\prime}\right) H\left(x_{n}-y_{n}\right), \quad E_{n}^{-}=-i \delta\left(x^{\prime}-y^{\prime}\right) H\left(y_{n}-x_{n}\right) .
$$

Here $H$ is the Heaviside function, $H(t)=1$ for $t>0$ and $H(t)=0$ for $t<0$, and we have used the notation $x=\left(x^{\prime}, x_{n}\right)$ and $y=\left(y^{\prime}, y_{n}\right)$ for points in $\mathbf{R}^{n}$. Note that $E_{n}^{+}-E_{n}^{-}=$ $i \delta\left(x^{\prime}-y^{\prime}\right)$ or, in Fourier integral form,

$$
\left(\boldsymbol{E}_{n}^{+}-\boldsymbol{E}_{n}^{-}\right)(u)=(2 \pi)^{-(4 n-2) / 4} \iiint e^{i\left\langle x^{\prime}-\boldsymbol{y}^{\prime}, \theta\right\rangle} i \sqrt{2 \pi} u(x, y) d x d y d \theta, \quad u \in C_{0}^{\infty}\left(\mathbf{R}^{n} \times \mathbf{R}^{n}\right) .
$$

Here $4 n-2=2 n+2(n-1)$ so the power of $2 \pi$ in front of the integral agrees with (2.4.1) and (3.2.14). For the phase function $\varphi(x, y, \theta)=\left\langle x^{\prime}-y^{\prime}, \theta\right\rangle$ the set $C_{\varphi}$ where $\varphi_{\theta}^{\prime}=0$ is defined by $x^{\prime}=y^{\prime}$, so it is parametrized by $x^{\prime}, x_{n}, y_{n}, \theta$ and the pullback of the Dirac measure in $\mathbf{R}^{n-1}$ under the map $(x, y, \theta) \rightarrow \varphi_{\theta}^{\prime}$ is the measure $d x^{\prime} d x_{n} d y_{n} d \theta$ on $C_{\varphi}$. The canonical relation defined by $\varphi$ is

$$
C_{n}=\left\{(x, \xi, y, \eta) ; x^{\prime}=y^{\prime}, \xi^{\prime}=\eta^{\prime} \neq 0, \xi_{n}=\eta_{n}=0\right\}
$$

and the bijection $C_{p} \rightarrow C_{n}$ gives the parametrization

$$
\left(x^{\prime}, x_{n}, y_{n}, \theta\right) \rightarrow\left(x^{\prime}, x_{n}, \theta, 0 ; x^{\prime}, y_{n}, \theta, 0\right) \in C_{n} .
$$

With these parameters and the trivialization of the Keller-Maslov line bundle $L$ given by $\varphi$ the principal symbol of $E_{n}^{+}-E_{n}^{-}$is 


$$
i \sqrt{2 \pi} \sqrt{d x^{\prime} d x_{n}} d y_{n} d \theta \in S_{1}^{(n-1) / 2}=S_{1}^{-\frac{1}{2}+2 n / 4}
$$

so $E_{n}^{+}-E_{n}^{-} \in I_{1}^{-\frac{1}{2}}\left(\mathbf{R}^{n} \times \mathbf{R}^{n}, C_{n}^{\prime}\right)$. It follows that $\chi E_{n}^{ \pm} \in I_{1}^{-\frac{1}{2}}\left(\mathbf{R}^{n} \times \mathbf{R}^{n}, C_{n}^{\prime}\right)$ if $\chi \in C^{\infty}\left(\mathbf{R}^{n} \times \mathbf{R}^{n}\right)$ vanishes in a neighborhood of the diagonal. In fact, if $x \neq y$ then either $E_{n}^{+}$or $E_{n}^{-}$vanishes in a neighborhood of $(x, y)$.

In particular we conclude that $W F^{\prime}\left(E_{n}^{\ddagger}\right)$ is contained in $C_{n}$ except over the diagonal in $\mathbf{R}^{n} \times \mathbf{R}^{n}$. Since $W F^{\prime}\left(\delta\left(x^{\prime}-y^{\prime}\right)\right) \subset C_{n}$ and $W F^{\prime}\left(H\left(x_{n}-y_{n}\right)\right) \subset C^{\prime}=\left\{(x, \xi, y, \eta) ; \xi^{\prime}=\eta^{\prime}=0\right.$, $\left.x_{n}=y_{n}, \xi_{n}=\eta_{n} \neq 0\right\}$, it follows from Theorem 2.5.10 that $W F^{\prime}\left(E_{n}^{+}\right) \subset\left(C^{\prime}+C_{n}\right) \cup C^{\prime} \cup C_{n}$. Over the diagonal in $\mathbf{R}^{n} \times \mathbf{R}^{n}$ this set is the same as the diagonal in $\left(T^{*}\left(\mathbf{R}^{n}\right) \backslash 0\right) \times\left(T^{*}\left(\mathbf{R}^{n}\right)\right.$ 0 ). If we note that $W F^{\prime}\left(D_{n} E_{n}^{+}\right)=W F^{\prime}(\delta(x-y))$ is equal to the diagonal, we find on the other hand that $W F^{\prime}\left(E_{n}^{+}\right)$must contain the diagonal in $\left(T^{*}\left(\mathbf{R}^{n}\right) \backslash 0\right) \times\left(T^{*}\left(\mathbf{R}^{n}\right) \backslash 0\right)$, and so we have proved

Proposition 6.1.2. Let $E_{n}^{+}$and $E_{n}^{-}$be the forward and backward fundamental solutions of $D_{n}=-i \partial / \partial x_{n}$ in $\mathbf{R}^{n}$. Then we have

(i) $W F^{\prime}\left(E_{n}^{ \pm}\right)$is the union of the diagonal in $T^{*}\left(\mathbf{R}^{n}\right) \backslash 0 \times T^{*}\left(\mathbf{R}^{n}\right) \backslash 0$ and the part of the canonical relation $C_{n}$ defined by (6.1.2) where $x_{n} \gtrless y_{n}$.

(ii) $\chi E_{n}^{ \pm} \in I_{1}^{-\frac{1}{2}}\left(\mathbf{R}^{n} \times \mathbf{R}^{n}, C_{n}^{\prime}\right)$ if $\chi \in C^{\infty}\left(\mathbf{R}^{n} \times \mathbf{R}^{n}\right)$ vanishes near the diagonal.

(iii) $E_{n}^{+}-E_{n}^{-} \in I_{1}^{-\frac{1}{2}}\left(\mathbf{R}^{n} \times \mathbf{R}^{n}, C_{n}^{\prime}\right)$ and a principal symbol is given by (6.1.4) where the parametrization is that of (6.1.3) and the Keller-Maslov line bundle is trivialized by means of the phase function $\left\langle x^{\prime}-y^{\prime}, \theta\right\rangle$.

Using (i) only we shall now prove Theorem 6.1.1 when $X=\mathbf{R}^{n}$ and $P=D_{n}$. First assume that $v \in \mathscr{E}^{\prime}\left(\mathbf{R}^{n}\right)$ and set $g=D_{n} v$. Then we have $v=E_{n}^{+} g=E_{n}^{-} g$. If $C_{n}^{+}$and $C_{n}^{-}$denote the parts of $C_{n}$ where $x_{n}>y_{n}$ and $x_{n}<y_{n}$ respectively, then by (i) in Proposition 6.1.2 and Theorem 2.5.14 it follows that

$$
W F(v) \subset\left(W F(g) \cup C_{n}^{+} W F(g)\right) \cap\left(W F(g) \cup C_{n}^{-} W F(g)\right)
$$

Consider now a distribution $u \in \mathscr{D}^{\prime}\left(\mathbf{R}^{n}\right)$ and set $D_{n} u=f$. Since the Hamilton field of $p(x, \xi)=\xi_{n}$ is the unit vector $e_{n}$ along the $x_{n}$-axis, Theorem 6.1 .1 will be proved for the operator $D_{n}$ if we show that $\left(x_{0}, \xi_{0}\right) \in W F(u) \backslash W F(f)$ implies $\left(x_{0}, \xi_{0}\right)+t e_{n} \in W F(u)$ for small $t$. Choose $\delta>0$ so small that $\left(x_{0}, \xi_{0}\right)+t e_{n} \notin W F(f)$ when $|t| \leqslant \delta$, and then $\varphi \in C_{0}^{\infty}\left(\mathbf{R}^{n}\right)$ with $\left|x-x_{0}\right| \leqslant \delta$ in $\operatorname{supp} \varphi$. If $v=\varphi u$ and $g=D_{n} v$, we have

$$
W F(g) \subset W F(\varphi f) \cup W F\left(u D_{n} \varphi\right),
$$

and $W F(\varphi f)$ contains no point of the form $\left(x_{0}, \xi_{0}\right)+t e_{n}$. If $\varphi\left(x_{0}\right)=1$ then $\left(x_{0}, \xi_{0}\right) \in W F(v)$ 
so it follows from (6.1.5) that $\left(x_{0}, \xi_{0}\right)+t e_{n}$ is in $W F(u)$ and over supp $D_{n} \varphi$ for some $t>0$ and some $t<0$. Hence $\left(x_{0}, \xi_{0}\right)+t e_{n} \in W F(u)$ for $|t| \leqslant \delta$.

In the general proof of Theorem 6.1.1 we may assume that $P$ is a first order operator, for if $Q$ is an elliptic pseudo-differential operator with positive principal part, homogeneous of degree $1-m$, then $P u=f$ implies $(Q P) u=Q f$ where $Q P$ has the same characteristics and bicharacteristic strips as $P$, and $W F(Q f)=W F(f)$. It is also sufficient to consider characteristics $\left(x_{0}, \xi_{0}\right)$ of $P$ where the Hamilton field $H_{p}$ does not have the direction of the cone axis $\left\{\left(x_{0}, \tau \xi_{0}\right) ; \tau>0\right\}$, for the bicharacteristic strip starting at $\left(x_{0}, \xi_{0}\right)$ is otherwise equal to the cone axis and this makes the assertion of Theorem 6.1.1 trivial. Using these conditions we shall now transform $P$ locally at $\left(x_{0}, \xi_{0}\right)$ to the operator $D_{n}$. The first step is an essentially classical construction of canonical coordinates (see Carathéodory [6, § 105-6]).

Proposition 6.1.3. Let $p_{1}, \ldots, p_{k}$ be real valued $C^{\infty}$ functions in a conic neighborhood of $\left(x_{0}, \xi_{0}\right) \in T^{*}(X) \backslash 0$ which are homogeneous of degree 1. Let $n=\operatorname{dim} X$. For the existence of a homogeneous canonical transformation $\chi(x, \xi)=\left(X_{1}(x, \xi), \ldots, X_{n}(x, \xi), \Xi_{1}(x, \xi), \ldots, \Xi_{n}(x, \xi)\right)$ from a conic neighborhood $U$ of $\left(x_{0}, \xi_{0}\right)$ to a conic neighborhood of $\left(0, \Xi_{0}\right) \in T^{* *}\left(\mathbf{R}^{n}\right) \backslash 0$ such that $p_{j}(x, \xi)=\Xi_{j}(x, \xi), j=1, \ldots, k$, it is then necessary and sufficient that

(i) $\left\{p_{i}, p_{j}\right\}=0$ in a neighborhood of $\left(x_{0}, \xi_{0}\right)$ for $i, j=1, \ldots, k$;

(ii) $H_{p}\left(x_{0}, \xi_{0}\right), \ldots, H_{p_{k}}\left(x_{0}, \xi_{0}\right)$ and the direction of the cone axis are linearly independent.

Here $\{p, q\}=H_{p} q$ denotes the Poisson bracket, and $H_{p}$ the Hamilton field.

Proof. Since Poisson brackets, Hamilton fields and the cone axis are all invariant under homogeneous canonical transformations, the necessity follows from the fact that $\left\{\Xi_{i}, \Xi_{j}\right\}=0, H_{\Xi_{i}}=\partial / \partial X_{i}$ with respect to the symplectic structure in $T^{*}\left(\mathbf{R}^{n}\right)$.

For the proof of sufficiency we first note that the symplectic form vanishes in the $k$-plane $B$ spanned by $H_{p_{1}}\left(x_{0}, \xi_{0}\right), \ldots, H_{p_{k}}\left(x_{0}, \xi_{0}\right)$ so $k \leqslant n$. If $p_{1}\left(x_{0}, \xi_{0}\right)=\ldots=p_{k}\left(x_{0}, \xi_{0}\right)=0$, then $d p_{1}, \ldots, d p_{k}$ vanish in the direction of the cone axis by Euler's relations for homogeneous functions. Hence the symplectic form vanishes in the plane which it spans with $B$ and so $k \leqslant n-1$ then. We shall now consider separately the construction of the coordinates $\Xi_{j}$ and $X_{j}$.

(a) Assume that $k=n$. Writing $\Xi_{j}(x, \xi)=p_{j}(x, \xi)$ we have to find $X_{i}$ homogeneous of degree 0 so that

$$
\begin{gathered}
H_{p_{j}}(x, \xi) X_{i}(x, \xi)=\delta_{i j} ; \quad i, j=1, \ldots, n \\
\left\{X_{i}, X_{j}\right\}=0 ; \quad i, j=1, \ldots, n .
\end{gathered}
$$

To do so we shall choose a conic $n$-dimensional manifold $S$ through $\left(x_{0}, \xi_{0}\right)$ which is transversal to $B$ and choose $X_{1}, \ldots, X_{n}$ equal to 0 on $S$ and satisfying (6.1.6). The differentials 
are then linearly independent so $S$ must be Lagrangean in view of (6.1.7). All these conditions can be fulfilled since the tangent plane of $S$ at $\left(x_{0}, \xi_{0}\right)$ can be taken as any Lagrangean plane containing the direction of the cone axis. (Cf. pp. 137-138 and 161-162 in Chap. III.) Since

$$
\left[H_{p_{i}}, H_{p_{j}}\right]=H_{\left\{p_{i}, p_{j}\right\}}=0
$$

in view of (i), the equations (6.1.6) have a unique local solution and it is clearly homogeneous of degree 0 . The equations (6.1.7) are valid on $S$ since $S$ is Lagrangean and follow in general by another application of the Jacobi identity,

$$
H_{p_{j}}\left\{X_{i}, X_{i^{\prime}}\right\}=-\left\{X_{i},\left\{X_{i^{\prime}}, p_{j}\right\}\right\}-\left\{X_{i^{\prime}},\left\{p_{j}, X_{i}\right\}\right\}=0 \text {. }
$$

(b) If $k<n$ we shall prove that one can choose $p_{k+1}=u$ so that (i) and (ii) remain valid for $p_{1}, \ldots, p_{k+1}$. Combined with (a) this will of course prove the proposition. Conditions (i) require that

$$
H_{p_{j}} u=0, j=1, \ldots, k
$$

and as above we can solve these equations with prescribed data on a conic manifold of codimension $k$ transversal to $B$. If the data are homogeneous of degree 1 the same will be true of $u$. Since the differential of $u$ at $\left(x_{0}, \xi_{0}\right)$ is unrestricted by this condition we can choose the data so that $d p_{1}, \ldots, d p_{k}, d u$ and $\xi d x$ are linearly independent, for the dimension $2 n-k$ of the manifold exceeds $k+1$. The proof is now complete.

The special case $k=1$ underlies the following

Proposition 6.1.4. Let $P \in L_{1}^{1}(X)$ have real and homogeneous principal part $p$ and assume that the Hamilton field $H_{p}$ at $\left(x_{0}, \xi_{0}\right) \in T^{*}(X) \backslash 0$ is not a tangent to the cone axis. Choose any homogeneous canonical transformation $\chi$ from an open conic neighborhood of $\left(x_{0}, \xi_{0}\right) \in T^{*}(X) \backslash 0$ to a conic neighborhood of some point $\left(X_{0}, \Xi_{0}\right) \in T^{*}\left(\mathbf{R}^{n}\right) \backslash 0$ such that $p$ is the composition of the coordinate function $\Xi_{n}$ and $\chi$. For any $\mu \in \mathbf{R}$ one can then find a Fourier integral operator $A \in I_{1}^{\mu}\left(X \times \mathbf{R}^{n}, \Gamma^{\prime}\right)$ such that.

(i) $\Gamma$ is a closed conic subset of the graph of $\chi$.

(ii) $\left(x_{0}, \xi_{0}, X_{0}, \Xi_{0}\right)$ is a non-characteristic point for $A$.

(iii) A transforms $P$ to $D_{n}$ locally in the sense that

$$
\left(x_{0}, \xi_{0}, X_{0}, \Xi_{0}\right) \notin W F^{\prime \prime}\left(P A-A D_{n}\right) .
$$

Proof. We start with any $A_{1} \in I_{1}^{\mu}$ which satisfies (i) and (ii). By the local version of Proposition 5.1.2 expressed by (5.1.2) we can then choose $B_{1} \in I_{1}^{-\mu}\left(\mathbf{R}^{n} \times X,\left(\Gamma^{-1}\right)^{\prime}\right)$ so that $\left(x_{0}, \xi_{0}\right) \notin W F\left(A_{1} B_{1}-I\right)$. (All operators can be taken properly supported.) If we set $Q=B_{1} P A_{1}$ then $P A_{1}-A_{1} Q=\left(I-A_{1} B_{1}\right) P A_{1}$, so 


$$
\left(x_{0}, \xi_{0}, X_{0}, \Xi_{0}\right) \notin W F^{\prime}\left(P A_{1}-A_{1} Q\right) .
$$

Since $A_{1}$ and $B_{1}$ have reciprocal principal symbols, it follows from the choice of $\chi$ that the principal symbol of $Q$ is $\xi_{n}$ in a conic neighborhood of $\left(X_{0}, \Xi_{0}\right)$. Thus we can choose $q \in L_{1}^{0}\left(\mathbf{R}^{n}\right)$ so that

$$
\left(X_{0}, \Xi_{0}\right) \notin W F\left(Q-D_{n}-q\right) .
$$

We shall now prove that there is an elliptic pseudo-differential operator $A_{2} \in L_{\mathbf{1}}^{0}\left(\mathbf{R}^{n}\right)$ such that

$$
\left(D_{n}+q\right) A_{2}-A_{2} D_{n} \in L^{-\infty} \text {. }
$$

Admitting this for a moment we set $A=A_{1} A_{2}$. That (i) and (ii) are valid is then obvious, and since

$$
P A-A D_{n}=\left(P A_{1}-A_{1} Q\right) A_{2}+A_{1}\left(\left(Q-D_{n}-q\right) A_{2}+\left(D_{n}+q\right) A_{2}-A_{2} D_{n}\right)
$$

we obtain (iii) from (6.1.9)-(6.1.11).

To solve (6.1.11) we rewrite the condition in the form

$$
\left[D_{n}, A_{2}\right]+q A_{2} \in L^{-\infty} .
$$

If $q^{0}$ is the principal symbol of $Q$ and $a^{0}$ the principal symbol of $A_{2}$ the vanishing of the principal symbol requires that

$$
i^{-1}\left\{\xi_{n}, a^{0}\right\}+q^{0} a^{0}=0,
$$

that is, $\partial a^{0} / \partial x_{n}=-i q^{0} a^{0}$. This equation is solved by

$$
a^{0}(x, \xi)=\exp \left(-i \int_{0}^{x_{n}} q^{0}\left(x^{\prime}, t, \xi\right) d t\right)
$$

which is an element of $S_{1}^{0}$ by Proposition 1.1.8. Choosing $A^{0}$ with principal symbol $a^{0}$ we can now successively choose $A^{j} \in L_{1}^{-j}\left(\mathbf{R}^{n}\right)$ so that for every $j$

$$
\left[D_{n}, A^{0}+\ldots+A^{j}\right]+q\left(A^{0}+\ldots+A^{j}\right)=R_{j} \in L_{1}^{-j-1} .
$$

In fact, this only requires that the principal symbol $a^{j}$ of $A^{j}$ satisfies the equation

$$
i^{-1} \partial a^{j} / \partial x_{n}+q^{0} a^{j}=-r_{j-1}^{0}
$$

where $r_{j-1}^{0}$ is the principal symbol of $R_{j-1}$. The solution

$$
a^{j}(x, \xi)=a^{0}(x, \xi) \int_{0}^{x_{n}}-i r_{j-1}^{0}\left(x^{\prime}, t, \xi\right) / a^{0}\left(x^{\prime}, t, \xi\right) d t
$$

is in $S_{1}^{-3}$ by Proposition 1.1.8 again. Following Proposition 5.1.1 we set $A_{2} \sim A^{0}+A^{1}+\ldots$ and have satisfied (6.1.12). 
Proof of Theorem 6.1.1. First recall that we have reduced the proof to the case $m=1$ and that the theorem has been proved for the operator $D_{n}$. So suppose that $m=1$ and let $\left(x_{0}, \xi_{0}\right) \in W F(u) \backslash W F(f)$. By definition of $W F(u)$ this implies that $p\left(x_{0}, \xi_{0}\right)=0$. Since $W F(u) \backslash W F(f)$ is a cone in $T^{*}(X) \backslash 0$ and an integral curve of $H_{p}$ tangent to a cone axis is contained in it, we may as already pointed out assume that $H_{p}\left(x_{0}, \xi_{0}\right)$ is linearly independent of the direction of the cone axis. We can then choose an operator $A$ according to Propositions 6.1.3, 6.1.4, and by the local form of Proposition 5.1.2 we can then construct an operator $B \in I_{1}^{-\mu}\left(\mathbf{R}^{n} \times X,\left(\Gamma^{-1}\right)^{\prime}\right)$ such that

$$
\left(x_{0}, \xi_{0}\right) \notin W F(A B-I), \quad\left(X_{0}, \Xi_{0}\right) \notin W F(B A-I) .
$$

Here $I$ stands for the identity operators in $X$ and in $\mathbf{R}^{n}$. Since

$$
B\left(P A-A D_{n}\right) B=B P(A B-I)-(B A-I) D_{n} B+B P-D_{n} B,
$$

it follows from (6.1.13) and (iii) in Proposition 6.1.4 that

$$
\left(X_{0}, \Xi_{0}, x_{0}, \xi_{0}\right) \notin W F^{\prime}\left(B P-D_{n} B\right) .
$$

We shall now consider $v=B u \in \mathscr{D}^{\prime}\left(\mathbf{R}^{n}\right)$. Since

$$
u=A v+(I-A B) u
$$

and $\left(x_{0}, \xi_{0}\right) \notin(I-A B) u$ by (6.1.13) we have $\left(X_{0}, \Xi_{0}\right) \in W F(v)$ for the opposite assumption would imply that $\left(x_{0}, \xi_{0}\right) \notin W F(u)$ in contradiction with our hypothesis. On the other hand,

$$
D_{n} v=D_{n} B u=B P u-\left(B P-D_{n} B\right) u
$$

so (6.1.14) and the hypothesis that $\left(x_{0}, \xi_{0}\right) \notin W F(P u)$ gives that $\left(X_{0}, \Xi_{0}\right) \notin W F\left(D_{n} v\right)$. From the special case of Theorem 6.1.1 already proved we now conclude that $W F(v)$ contains a neighborhood of $\left(X_{0}, \Xi_{0}\right)$ on the bicharacteristic curve of $D_{n}$ through $\left(X_{0}, \Xi_{0}\right)$, so it follows that $\chi W F(u)$ must contain this neighborhood. The invariance of the notion of bicharacteristic curve under canonical transformation therefore shows that $W F(u)$ contains a neighborhood of $\left(x_{0}, \xi_{0}\right)$ in the bicharacteristic of $P$ through this point, which proves the theorem.

In section 6.3 we shall need a more precise version of Theorem 6.1.1 which takes into account differentiability of finite order. To state it we need some terminology and notations. Again we recall that $H_{(s)}(X)$ denotes the set of all $u \in \mathscr{D}^{\prime}(X)$ such that $A u \in L_{\mathrm{Ioc}}^{2}(X)$ for all properly supported $A \in L_{1}^{s}(X)$, and that we have $B u \in H_{(s-m)}(X)$ if $u \in H_{(s)}(X)$ and $B \in L_{\varrho}^{m}(X), \varrho>\frac{1}{2}$. We now modify (2.5.2) by writing

$$
W F_{(s)}(u)=\bigcap \operatorname{char}(A)
$$


where the intersection is taken over all properly supported $A \in L_{1}^{0}$ with $A u \in H_{(s)}$, and char $(A)$ denotes the characteristics of $A$. (It would be equivalent to take $A \in L_{1}^{s}$ with $\left.A u \in L_{1 \mathrm{lo} .}^{2}\right)$ If $W F(A) \cap W F_{(s)}(u)=\varnothing$ and $A \in L_{\varrho}^{\mu}(X)$ is properly supported, $\varrho>\frac{1}{2}$, it follows then that $A u \in H_{(s-\mu)}$. Another way of defining $W F_{(s)}(u)$ is that $\left(x_{0}, \xi_{0}\right) \notin W F_{(s)}(u)$ if and only if we can write $u=u_{1}+u_{2}$ where $u_{1} \in H_{(s)}(X)$ and $\left(x_{0}, \xi_{0}\right) \notin W F\left(u_{2}\right)$. The simple proof is left for the reader.

Let $A \in \mathscr{D}^{\prime}(X \times Y)$ and assume that $W F(A) \subset\left(T^{*}(X) \backslash 0\right) \times\left(T^{*}(Y) \backslash 0\right)$. Also assume that for the corresponding operator we have

Then it follows that

$$
A\left(H_{(s)}(Y) \cap \mathscr{E}^{\prime}(Y)\right) \subset H_{(s-\mu)}(X)
$$

$$
W F_{(s-\mu)}(A u) \subset W F^{\prime}(A) W F_{(s)}(u), u \in \mathscr{E}^{\prime}(Y) .
$$

In fact, we can write $u=u_{1}+u_{2}$ with $u_{1} \in H_{(s)}$ and $W F\left(u_{2}\right)$ close to $W F_{(s)}(u)$, and since $A u_{1} \in H_{(s-\mu)}$ by (6.1.15) this allows us to deduce (6.1.16) from Theorem 2.5.14. Note that Theorem 4.3.1 gives (6.1.15) if $A$ is a Fourier integral operator of order $\mu$ corresponding to a canonical transformation from $T^{*}(Y) \backslash 0$ to $T^{*}(X) \backslash 0$. We also have (6.1.15) with $\mu=0$ if $X=Y=\mathbf{R}^{n}$ and $A=E_{n}^{ \pm}$, defined in Proposition 6.1.2, for $A u$ is convolution by a measure.

In view of the preceding remarks we obtain by obvious modifications of the proof of Theorem 6.1.1

THEOREM 6.1.1'. Assume that $P \in L_{1}^{m}(X)$ is properly supported and has a real principal part $p$ which is homogeneous of degree $m$. If $u \in \mathscr{D}^{\prime}(X)$ and $P u=f$, it follows that $W F_{(s+m-1)}(u) \backslash$ $W F_{(s)}(f)$ is contained in $p^{-1}(0)$ and is invariant under the Hamiltonian vector field $H_{p}$. Here $s$ is any real number.

\subsection{Construction of solutions with singularities}

The main result of this section shows that Theorems 6.1.1 and 6.1.1' cannot be improved at least when the bicharacteristics of $P$ are well behaved:

THEOREM 6.2.1. Assume that $P \in L_{1}^{m}(X)$ is properly supported and has a real principal part $p$ which is homogeneous of degree $m$. Let $I \subset \mathbf{R}$ be an open interval and $\gamma: I \rightarrow T^{*}(X) \backslash 0$ a map defining a bicharacteristic strip which remains injective after projection to the cosphere bundle. Denote by $\Gamma$ the closed conic hull of $\gamma(I)$ and by $\Gamma^{\prime}$ the limit points, that is, the intersection of the closed conic hull of $\gamma\left(I \backslash I_{0}\right)$ when $I_{0}$ runs over all compact intervals contained in I. For any $s \in \mathbf{R}$ one can then find $u$ so that $u \in H_{(t)}(X)$ for every $t<s$ and

$$
W F(P u) \subset \Gamma^{\prime}, \quad W F(u) \backslash \Gamma^{\prime}=W F_{(s)}(u) \backslash \Gamma^{\prime}=\Gamma \backslash \Gamma^{\prime} .
$$


Note that $\Gamma^{\prime}$ is empty precisely when $\gamma$ composed with the projection into $X$ is a proper map $I \rightarrow X$. Then we have

$$
P u \in C^{\infty}, \quad W F(u)=W F_{(s)}(u)=\Gamma .
$$

Proof. As in section 6.1 we may assume that $m=1$. We shall begin by proving a less precise result where $I$ is replaced by a compact subinterval $I_{0}$ and $\Gamma$ resp. $\Gamma^{\prime}$ by conic neighborhoods of $\gamma\left(I_{0}\right)$ resp. $\gamma\left(\partial I_{0}\right)$. There is of course nothing to prove if $\gamma(I)$ is a ray in $T^{*}(X) \backslash 0$ so we may assume that $\gamma$ is never tangential to the cone axis. If $t_{0}$ is a point in $I_{0}$ we can therefore choose a $n-1$ dimensional conic submanifold $N_{0}$ of $N=$ $\left\{(x, \xi) \in T^{*}(X) \backslash 0, p(x, \xi)=0\right\}$ such that the symplectic form vanishes in $N_{0}, \gamma\left(t_{0}\right) \in N_{0}$ and $H_{p}\left(\gamma\left(t_{0}\right)\right.$ ) is not tangent to $N_{0}$. (If $p(x, \xi)=\xi_{n}$ we can take for $N_{0}$ a plane $x=$ constant, $\xi_{n}=0$ so it follows from Proposition 6.1.3 that such a choice of $N_{0}$ is always possible.) If $(t, n) \rightarrow \varphi(t, n)$ is the Hamilton flow of $p$, that is, $\varphi(t, n)$ is the value for the parameter value $t$ of the solution of the Hamilton equations starting at $n$ when $t=0$, it is clear that there is a closed conic neighborhood $V_{0} \subset N_{0}$ of $\gamma\left(t_{0}\right)$ such that

$$
(t, n) \rightarrow \varphi\left(t-t_{0}, n\right)
$$

is injective on a neighborhood of $I_{0} \times V_{0}$. The range is therefore a closed conic subset $\Lambda$ of a conic manifold which is Lagrangean since $n \rightarrow \varphi(t, n)$ is a canonical transformation and $H_{p}$ is orthogonal to the tangent plane of $N_{0}$ by virtue of the fact that $p=0$ on $N_{0}$. It is clear that $p=0$ on $\Lambda$, and we have $\gamma\left(I_{0}\right)=\varphi\left(I_{0}, \gamma\left(t_{0}\right)\right) \subset \Lambda$.

Let $I_{0}^{\prime}$ be an open interval with closure contained in the interior of $I_{0}$ and set $\Lambda^{\prime}=$ $\varphi\left(I_{0} \backslash I_{0}^{\prime}, V_{0}\right)$. Since $H_{p}$ pulled back to $I_{0} \times V_{0}$ is $\partial / \partial t$, the hypothesis (5.3.10) is fulfilled in $\Lambda$ (compare the proof of Proposition 6.1.4). We can therefore choose $u \in I_{1}^{\mu}(X, \Lambda), \mu=$ $-s-n / 4$, so that $P u \in I_{1}^{\mu}\left(X, \Lambda^{\prime}\right)$ and the principal symbol of $u$ is prescribed on $V_{0}$ in the class $S_{1}^{\mu+n / 4}\left(V_{0}, L \otimes \Omega_{1}\right)$. If we choose it non-characteristic at $\gamma\left(t_{0}\right)$ it is non-characteristic at $\gamma\left(I_{0}^{\prime}\right)$. Taking Theorem 5.4.1 into account we conclude that $u \in H_{(t)}$ if $t<s$ and that

$$
W F(P u) \subset \Lambda^{\prime} ; \quad \gamma\left(I_{0}\right) \backslash \Lambda^{\prime} \subset W F_{(s)}(u) \subset W F(u) \subset \Lambda .
$$

By using symbols of type $\varrho$ with $2 / 3<\varrho<1$ and Lemma 6.5 .9 below it is easy to modify the preceding arguments so that $W F^{\prime}(u)=\gamma\left(I_{0}\right)$ and $W F(P u) \subset \gamma\left(\partial I_{0}\right)$. However, to obtain the full result including the precise $H_{(s)}$ regularity we shall use a more functional analytic argument combined with a modification of the preceding construction where $u$ depends on a parameter.

Let $F$ be the set of all $u \in \mathscr{D}^{\prime}(X)$ such that $u \in H_{(t)}(X)$ when $t<s, W F(P u) \subset \Gamma^{\prime}$ and $W F(u) \subset \Gamma$. The statement in the theorem is that $W F_{(s)}(u) \supset \Gamma \backslash \Gamma^{\prime}$ for some $u \in F$. 
Following section 2.5 we introduce in $F$ the weakest topology making the following maps continuous:

$$
F \hookrightarrow H_{(t)}, t<s ; F \ni u \rightarrow A u \in C^{\infty}(X) \text { and } F \ni u \rightarrow B P u \in C^{\infty}(X)
$$

where $A$ and $B$ are properly supported pseudo-differential operators with $W F(A) \cap \Gamma=\varnothing$ and $W F^{\prime}(B) \cap \Gamma^{\prime}=\varnothing$. It is clear that $F$ is a Fréchet space for we need only consider countably many choices of $t, A$ and $B$, and the completeness is obvious.

Lемма 6.2.2. If $T$ is a properly supported pseudo-differential operator of order s which is non-characteristic at some point $\left(x_{0}, \xi_{0}\right) \in \Gamma \backslash \Gamma^{\prime}$, then $\left\{u \in F ; T u \in L_{L_{100}^{2}}^{2}\right.$ is of the first category.

Admitting the lemma for a moment we can complete the proof of Theorem 6.2.1. To do so we choose a countable fundamental family of conic neighborhoods $V_{1}, V_{2}, \ldots$ of points in $\Gamma \backslash \Gamma^{\prime}$ and for every $j$ an operator $T_{j}$ as in the lemma with $W F\left(T_{j}\right) \subset V_{j}$. In view of the lemma it follows that $T_{j} u \notin L_{\mathrm{loc}}^{2}(X)$ for every $j$ if $u \in F$ and $u$ avoids a set of the first category. But this implies that $W F_{(s)}(u) \supset \Gamma \backslash \Gamma^{\prime}$ for most $u \in F$, so Theorem 6.2.1 is proved.

Proof of Lemma 6.2.2. If the assertion were false we would by the closed graph theorem have a continuous map $u \rightarrow T u$ from $F$ to $L_{\text {loc }}^{2}$. Let $\chi \in C_{0}^{\infty}(X), \chi\left(x_{0}\right) \neq 0$. The continuity implies that

$$
\|\chi T u\| \leqslant C\left(\left\|B_{1} u\right\|+\left\|B_{2} u\right\|+\left\|B_{3} P u\right\|\right), u \in F,
$$

where the norm is $L^{2}$ norm, and $B_{1}, B_{2}, B_{3}$ are pseudo-differential operators with kernels of compact support, $B_{1}$ is of order $t<s, W F\left(B_{2}\right) \cap \Gamma=\varnothing$ and $W F\left(B_{3}\right) \cap \Gamma^{\prime}=\varnothing$. We now return to the construction which led to (6.2.2). If we choose $I_{0}$ sufficiently large and $V_{0}$ sufficiently narrow, then

$$
\Lambda^{\prime} \cap W F^{\prime}\left(B_{3}\right)=\varnothing, \Lambda \cap W F\left(B_{2}\right)=\varnothing,\left(x_{0}, \xi_{0}\right) \in \gamma\left(I_{0}\right) \backslash \Lambda^{\prime} .
$$

Hence we have by (6.2.2)

$$
B_{3} P u \in C^{\infty}, B_{2} u \in C^{\infty}, B_{1} u \in L^{2}, \chi T u \notin L^{2} .
$$

This would contradict (6.2.3) if $u$ were in $F$ which is not the case though. To finish the proof we have to give a suitable approximation of $u$ by smooth functions.

Let $\psi \in C^{\infty}\left(T^{*}(X)\right)$ be equal to 1 and 0 respectively at $(x, \xi)$ when $|\xi|<1$ and $|\xi|>2$. Here $|\xi|$ is defined with respect to some Riemannian metric in $X$. Set $\psi_{\varepsilon}(x, \xi)=\psi(x, \xi / \varepsilon)$. These functions form a bounded set in $S_{1}^{0}$ as $0<\varepsilon \leqslant 1$ (see section 1.1). We now reconsider the construction of the distribution $u$ in (6.2.2) with the initial data for the principal symbol on $V_{0}$ multiplied by $\psi_{\varepsilon}$. This gives functions $u_{\varepsilon} \in C^{\infty}$ such that $u_{\varepsilon} \rightarrow u$ as $\varepsilon \rightarrow 0$ and 
since bounds independent of $\varepsilon$ are obtained at every step in the construction, (6.2.4) is replaced by boundedness of $B_{3} P u_{\varepsilon}, B_{2} u_{\varepsilon}$ in $C^{\infty}$ and of $B_{1} u_{\varepsilon}$ in $L^{2}$. Application of (6.2.3) therefore gives a uniform bound for $\left\|\chi T u_{\varepsilon}\right\|$ which implies that $\|\chi T u\|<\infty$ in contradiction with (6.2.4). This completes the proof.

Remark 1. Note that in the construction of $u_{\varepsilon}$ a symbol depending on $\varepsilon$ is considered to be of order $\sigma$ say only if it is in a bounded set in $S_{1}^{\sigma}$ when $0<\varepsilon<1$.

Remark 2. Since the assertion is obvious when $P=D_{n}$ the first part of the proof could be replaced by a semi-global version of Proposition 6.1.4. (See also Lemma 6.6.3.)

For large classes of operators Theorems 6.1.1 and 6.2.1 combined give a complete answer to the question what regularity properties of $u$ can be inferred from some known regularity of $u$ and of $P u$ :

THEOREM 6.2.3. Let $P \in L_{1}^{m}(X)$ be a properly supported operator with real and homogeneous principal part $p$. Assume that every bicharacteristic curve of $P$ is proper, that is, belongs to a compact set in $X$ only for a compact subset of the parameter interval. Let $F_{1}, F_{2}$ be two closed conic subsets of $T^{*}(X) \backslash 0$. Then we have

$$
u \in \mathscr{D}^{\prime}(X), W F(u) \subset F_{1}, W F(P u) \subset F_{2} \Rightarrow W F(u) \subset F
$$

if and only if $F \supset F_{1} \cap F_{2}$ and for every bicharacteristic strip $\Gamma$ the set $F$ contains every component $\Gamma_{0}$ of $\Gamma \backslash\left(F_{1} \cap F_{2}\right)$ in $\Gamma$ such that $\Gamma_{0} \subset F_{1}$.

Proof. We may assume that $F_{2} \subset F_{1}$ without restriction. If $(x, \xi) \in W F(u) \backslash F_{2}$ then the bicharacteristic strip $\Gamma$ through $(x, \xi)$ will by Theorem 6.1 .1 belong to $W F(u)$ until it meets $F_{2}$. Thus $(x, \xi)$ is in a component $\Gamma_{0}$ of $\Gamma \backslash F_{2}$ which satisfies the conditions in the theorem. On the other hand, for such a component $\Gamma_{0}$ it follows from Theorem 6.2.1 that one can find $u \in \mathscr{D}^{\prime}(X)$ with $W F(P u) \subset F_{2}$ and $\Gamma_{0} \subset W F(u) \subset F_{1}$. In fact, since the complete bicharacterstic strip $\Gamma$ containing $\Gamma_{0}$ has a proper projection in $X$, the only limit points of the conic hull of $\Gamma_{0}$ to consider in Theorem 6.2.1 belong to $F_{2}$.

By the principle of condensation of singularities we also obtain from Theorem 6.2.1:

THEOREM 6.2.4. Let $P \in L_{1}^{m}(X)$ be properly supported and have a real and homogeneous principal symbol. Denote by $K$ the closed conic hull of a family of bicharacteristic strips each of which has a proper projection in $X$. Then there exists a distribution $u$ in $X$ such that $W F(u)=K$ and $P u \in C^{\infty}$.

The details of the obvious proof are left for the reader. 


\subsection{Existence theorems}

In this section we shall study the existence of solutions of a pseudodifferential equa. tion $P u=f$ by combining abstract functional analysis with the results of sections 6.1 and 6.2 applied to the adjoint ${ }^{t} P$. At first we only consider solvability on compact subsets.

THEOREM 6.3.1. Assume that $P \in L_{1}^{m}(X)$ is properly supported and has a real principal part $p$ which is homogeneous of degree $m$. Let $K$ be a compact subset of $X$ such that no complete bicharacteristic curve is contained in $K$. Then it follows that

$$
N(K)=\left\{v \in \mathscr{E}^{\prime}(K),{ }^{t} P v=0\right\}
$$

is a finite dimensional subspace of $C_{0}^{\infty}(K)$ orthogonal to $P \mathscr{D}^{\prime}(X)$. If $f \in H_{(s)}(X)$ for some $s \in \mathbf{R}$ resp. $f \in C^{\infty}(X)$, and if $f$ is orthogonal to $N(K)$, then one can find $u \in H_{(s+m-1)}(X)$ resp. $u \in C^{\infty}(X)$ so that $P u=f$ in a neighborhood of $K$.

Proof. That $N(K) \subset C^{\infty}$ follows from Theorem 6.1.1 since for any $v \in N(K)$ and $(x, \xi) \in W F(v)$ the bicharacteristic strip starting at $(x, \xi)$ must remain over $K$. By the closed graph theorem the $L^{2}$ topology in $N(K)$ is equivalent to the $C^{\infty}$ topology which shows that the unit ball in the $L^{2}$ topology is compact so that $\operatorname{dim} N(K)<\infty$.

The hypotheses of the lemma are also fulfilled if $K$ is replaced by a sufficiently small compact neighborhood $K^{\prime}$. To prove this we may assume that $m=1$ and can then consider the bicharacteristic strips as curves in the cosphere bundle. Since this is compact over $K^{\prime}$ we would obtain a bicharacteristic strip staying over $K$ for all values of the parameters if there is one over $K^{\prime}$ for every $K^{\prime}$. This proves the statement. Since $\operatorname{dim} N\left(K^{\prime}\right)$ decreases with $K^{\prime}$ it is also clear that $N\left(K^{\prime}\right)=N(K)$ if $K^{\prime}$ is sufficiently close to $K$.

Let \|\|$_{(\sigma)}$ denote a norm which defines the $H_{(\sigma)}$ topology for distributions with support in an arbitrary fixed compact subset of $X$. Since $v \in \mathscr{E}^{\prime}(K),{ }^{t} P v \in H_{(\sigma)}$ implies $v \in H_{(\sigma+m-1)}$ by 'Theorem 6.1.1', it follows from the closed graph theorem that

$$
\|v\|_{(\sigma+m-1)} \leqslant C\left(\|t P v\|_{(\sigma)}+\|v\|_{(\sigma+m-2)}, v \in C_{0}^{\infty}(K) .\right.
$$

Let $V$ be a supplementary space of $N(K)$ in $H_{(\sigma+m-1)}(X) \cap \mathscr{E}^{\prime}(K)$. Then there is another constant $C_{1}$ such that

$$
\|v\|_{(\sigma+m-1)} \leqslant C_{1}\|t P v\|_{(\sigma)}, v \in V \cap C_{0}^{\infty}(K) .
$$

In fact, if this were false we could select a sequence $v_{j} \in V$ with

$$
\left\|v_{j}\right\|_{(\sigma+m-1)}=1, \quad\left\|t P v_{j}\right\|_{(\sigma)} \rightarrow 0 \text {. }
$$

A weakly convergent subsequence must converge strongly in $H_{(\sigma+m-2)}$ to a limit $v \in V$ with 
${ }^{t} P v=0$, and $1 \leqslant C\|v\|_{(\sigma+m-2)}$ by (6.3.2). Hence $v$ is a non-zero element of $N(K)$ belonging to $V$ which is a contradiction.

If $f \in H_{(s)}$ is orthogonal to $N(K)$ we set $\sigma=1-m-s$ and have by (6.3.3) for some $C$

$$
|\langle f, v\rangle| \leqslant C\left\|^{t} P v\right\|_{(\sigma)}, v \in C_{0}^{\infty}(K)
$$

for this is true if $v \in C_{0}^{\infty}(K) \cap V$ and neither side changes if an element of $N(K)$ is added to $v$. By the Hahn-Banach theorem it follows that the linear form ${ }^{t} P v \rightarrow\langle f, v\rangle, v \in C_{0}^{\infty}(K)$, can be extended to a distribution $u \in H_{(-\sigma)}=H_{(s+m-1)}$. Since

$$
\langle f, v\rangle=\left\langle u,{ }^{t} P v\right\rangle, v \in C_{0}^{\infty}(K)
$$

we have $P u=f$ in the interior of $K$. If we apply this conclusion to a suitable neighborhood $K^{\prime}$ of $K$ we obtain $P u=f$ in a neighborhood of $K$.

To discuss the $C^{\infty}$ case we denote by $C^{\infty}(K)$ the quotient of $C^{\infty}(X)$ by the subspace of functions vanishing of infinite order on $K$. The dual space of this Fréchet space is $\mathscr{E}^{\prime}(K)$. To show that the range of the map $C^{\infty}(X) \rightarrow C^{\infty}(K)$ defined by $P$ is the orthogonal complement of $N(K)$ we have to show (see e.g. Dieudonné-Schwartz [35, Th. 7]) that ${ }^{t} P \mathscr{E}^{\prime}(K)$ is weakly closed in $\mathscr{E}^{\prime}(X)$, or equivalently by a theorem of Banach (see Bourbaki [31, Ch. III, Th. 5]) that the intersection of ${ }^{t} P \mathscr{E}^{\prime}(K)$ and the unit ball in $H_{(\sigma)} \cap \mathscr{E}^{\prime}\left(K_{1}\right)$ is weakly closed for every real $\sigma$ and compact $K_{1} \subset X$. Now $v \in \mathscr{E}^{\prime}(K),{ }^{t} P v \in H_{(\sigma)}$ implies $v \in H_{(\sigma+m-1)}$ and by (6.3.3) $v=v_{1}+v_{2}$ were $v_{1} \in N(K)$ and $\left\|v_{2}\right\|_{(\sigma+m-1)} \leqslant C_{1}$. Since the set of such $v_{2} \in \mathscr{E}^{\prime}(K)$ is weakly compact and ${ }^{t} P v={ }^{t} P v_{2}$, the assertion is proved.

Remark 1. When $K$ consists of a point $x_{0}$ we conclude that there is local solvability of the equation $P u=f$ at $x_{0}$, with $u \in C^{\infty}$ if $f \in C^{\infty}$, provided that no bicharacteristic strip stays over $x_{0}$.

Remark 2. The condition on the bicharacteristics made in Theorem 6.3.1 is merely sufficient and in no way necessary for the conclusion to be valid. For example, if $P$ has constant coefficients our assumption means that the real characteristics are simple but the conclusion is always valid in the $C^{\infty}$ case and may hold in the $H_{(s)}$ spaces also, for example if $P$ is the heat equation, even if there are multiple characteristics. Even when the characteristics are simple the condition is not necessary in the variable coefficient case. For example, the conclusions of Theorem 6.3.1 are valid for

$$
P=x_{2} \partial / \partial x_{1}-x_{1} \partial / \partial x_{2}+c
$$

in $X=\left\{\left(x_{1}, x_{2}\right) ; \quad 1<x_{1}^{2}+x_{2}^{2}<2\right\}$ if $c$ is a real constant $\neq 0$, but the circles $x_{1}^{2}+x_{2}^{2}=r^{2}$ are bicharacteristic curves. Thus the lower order terms may in general be essential. However, 14-722909 Acta mathematica 128. Imprimé le 24 Mars 1972 
they are irrelevant when the hypotheses of Theorem 6.3.1 are fulfilled, and we introduce a terminology which refers to this fact:

Definition 6.3.2. Let $P \in L_{1}^{m}(X)$ be a properly supported pseudo-differential operator. We shall say that $P$ is of real principal type in $X$ if $P$ has a real homogeneous principal part $p$ of order $m$ and no complete bicharacteristic strip of $P$ stays over a compact set in $X$.

We shall now discuss global solvability of the equation $P u=f$. The best results refer to this equation $\bmod C^{\infty}$.

THEOREM 6.3.3. Let $P$ be of real principal type in the manifold $X$. Then the following conditions are equivalent:

(a) $P$ defines a surjective map from $\mathscr{D}^{\prime}(X)$ to $\mathscr{D}^{\prime}(X) / C^{\infty}(X)$.

(b) For every compact set $K \subset X$ there is another compact set $K^{\prime} \subset X$ such that

$$
u \in \mathscr{E}^{\prime}(X) \text {, sing supp }{ }^{t} P u \subset K \Rightarrow \text { sing supp } u \subset K^{\prime}
$$

(c) For every compact set $K \subset X$ there is another compact set $K^{\prime} \subset X$ such that $K^{\prime}$ contains any interval on a bicharacteristic curve with respect to $P$ having both end points in $K$.

Proof. $(\mathrm{b}) \Rightarrow$ (c) with the same $K^{\prime}$ by Theorem 6.2.1. Using Theorem 6.1.1 we shall also prove that $(\mathrm{c}) \Rightarrow(\mathrm{b})$. In doing so we may assume that $P$ is of order 1 since we can multiply $P$ by an elliptic operator of order $1-m$ without affecting these conditions. This has the advantage that the bicharacteristic strips can be considered as integral curves of a vector field on the cosphere bundle, and the fibers of this bundle are compact. Assuming that $u \in \mathscr{E}^{\prime}(X),(x, \xi) \in W F(u)$, we shall show that if $x \notin K^{\prime}$ there is a contradiction. By Theorem 6.1.1 the bicharacteristic strip through $(x, \xi)$ stays in $W F(u)$ until it reaches a point lying over $K$. In view of (c) and the assumption that $x \notin K^{\prime}$ at least one half ray $\gamma$ of the bicharacteristic strip starting at $(x, \xi)$ contains no point lying above $K$ so $\gamma \subset W F(u)$. Choose $\left(x_{0}, \xi_{0}\right)$ so that its class in the cosphere bundle is a limit point of $\gamma$ at inifinity, which is possible since $\gamma$ lies over the compact set supp $u$. Then the entire bicharacteristic strip with initial data $\left(x_{0}, \xi_{0}\right)$ must stay over supp $u$, which contradicts the hypothesis that $P$ is of principal type.

That (b) together with the fact that $K^{\prime}$ may be taken empty when $K$ is empty implies (a) is a result of pure functional analysis (Hörmander [46, Theorem 1.2.4]), so it just remains to show that (a) $\Rightarrow$ (c). Assume that (c) is not valid. For some compact set $K \subset X$ we can then find a sequence of compact intervals $I_{1}, I_{2}, \ldots$ on bicharacteristic strips with end points lying over $K$ and points $\left(x_{i}, \xi_{j}\right) \in I_{j}$ with $x_{j} \rightarrow \infty$ in $X$. We may assume that the intervals $I_{j}$ are disjoint even when considered in the cosphere bundle. Let $\left(y_{j}, \eta_{j}\right)$ be one end point of $I_{j}$ 
and let $\Gamma_{j}$ and $\Gamma_{j}^{\prime}$ be defined as in Theorem 6.2.1 relative to the interval of bicharacteristic strip bounded by $\left(y_{j}, \eta_{j}\right)$ and $\left(x_{j}, \xi_{j}\right)$. We take a sequence $s_{j} \rightarrow-\infty$ and then use Theorem 6.2.1 to determine $u_{j} \in \mathscr{D}^{\prime}(X)$ such that

$$
W F_{(s)}\left(u_{j}\right)=W F\left(u_{j}\right)=\Gamma_{j}, W F\left(P u_{j}\right)=\Gamma_{j}^{\prime} .
$$

We can then write $P u_{j}=f_{j}+g_{j}$ where $W F\left(f_{j}\right)$ and $W F^{\prime}\left(g_{j}\right)$ is the ray defined by $\left(x_{j}, \xi_{j}\right)$ and $\left(y_{j}, \eta_{j}\right)$ respectively. In doing so we can take the support of $f_{j}$ so close to $x_{j}$ that the supports of the distributions $f_{j}$ are locally finite. Set

$$
f=\sum f_{j}
$$

We claim that $P u-f$ is not in $C^{\infty}$ for any $u \in \mathscr{D}^{\prime}(X)$, thus (a) is not valid. In fact, given $u$ we can choose $s$ so large negative that

$$
W F_{(s)}(u) \cap \pi^{-1} K=\varnothing .
$$

When $s_{j} \leqslant s$ it follows that $W F_{(s)}\left(u-u_{j}\right)$ contains a neighborhood of $\left(y_{j}, \eta_{j}\right)$ on $I_{j}$ but not the other end point of $I_{\hat{j}}$. In view of Theorem $6.1 .1^{\prime}$ this shows that $W F_{(s)}\left(P\left(u-u_{j}\right)\right)$ must meet the interior of $I_{j}$. However,

$$
P\left(u-u_{j}\right)=P u-f+\sum_{k \neq j} f_{k}-g_{j}
$$

and $I_{j}$ does not meet the wave front set of the sum. Hence $I_{j}$ meets $W F_{(s)}(P u-f)$ which proves that $P u-f$ is not in $C^{\infty}$.

Remark. Note that the proof only requires a local version of Theorem 6.2.1.

A criterion for the existence of genuine solutions is given in the following theorem.

THE OREM 6.3.4. Let $P$ be a pseudo-differential operator of real principal type satisfying one of the equivalent conditions in Theorem 6.3.3. The following conditions are then equivalent:

(a) The equation $P u=f$ has a solution $u \in \mathscr{D}^{\prime}(X)$ for every $f \in \mathscr{D}^{\prime}(X)$ such that $\langle f, v\rangle=0$ for every $v \in C_{0}^{\infty}(X)$ with ${ }^{t} P v=0$

(b) The equation $P u=f$ has a solution $u \in C^{\infty}(X)$ for every $f \in C^{\infty}(X)$ such that $\langle f, v\rangle=0$ for all $v \in C_{0}^{\infty}(X)$ with ${ }^{t} P v=0$.

(c) For every compact set $K \subset X$ there exists a compact set $K^{\prime} \subset X$ such that if $v \in \mathscr{E}^{\prime}(X)$ and supp ${ }^{t} P v \subset K$ it follows that ${ }^{t} P v={ }^{t} P w$ for some $w \in \mathscr{E}^{\prime}(X)$ with supp $w \subset K{ }^{\prime}$.

(d) Same condition but with $v, w \in C_{0}^{\infty}$.

Proof. That (b) $\Rightarrow$ (a) follows from the hypothesis. For the implication (c) $\Rightarrow$ (b) we refer to Malgrange [48, pp. 328-329]; a special case of the argument was used in proving. 
Theorem 6.3.1 above. It is quite obvious that $(\mathrm{d}) \Rightarrow(\mathrm{c})$. For by $(\mathrm{b})$ in Theorem 6.3.3 there is a compact set $K^{\prime}$ such that

$$
v \in \mathscr{E}^{\prime}(X) \text {, sing supp }{ }^{\mathrm{t}} P v \subset K \Rightarrow \text { sing } \operatorname{supp} v \subset K^{\prime} \text {. }
$$

Choose $\chi \in C_{0}^{\infty}(X)$ equal to 1 in a neighborhood of $K^{\prime}$ and set $v_{1}=\chi v, v_{2}=(1-\chi) v$. Then supp $v_{1} \subset \operatorname{supp} \chi$ and if $\operatorname{supp}^{t} P v \subset K$ we obtain

$$
\text { supp }{ }^{t} P v_{2} \subset \operatorname{supp}{ }^{t} P v \cup \operatorname{supp}{ }^{t} P v_{1} \subset K_{1}
$$

where $K_{1}$ is a fixed compact set. Since $v_{2} \in C_{0}^{\infty}$ it follows from (d) that ${ }^{t} P v_{2}={ }^{t} P v_{3}$ for some $v_{3} \in C_{0}^{\infty}$ with supp $v_{3}$ in a fixed compact set $K_{1}{ }^{\prime}$. We have ${ }^{t} P v={ }^{t} P\left(v_{1}+v_{3}\right)$ and supp $\left(v_{1}+v_{3}\right)$ belongs to a fixed compact set.

Assuming now that (a) is valid we shall prove (d). Let $\mathscr{F}$ be the set of all $f \in C^{\infty}(X)$ satisfying the conditions in (a). This is a closed subspace of $C^{\infty}(X)$ and therefore a Fréchet space. Note that if $K$ is a compact set in $X$ and $f \in C^{\infty}(X)$ is orthogonal to $N(K)$, then one can find $g \in \mathscr{F}$ equal to $f$ in a neighborhood of $K$. Here $N(K)$ is the finite dimensional space in Theorem 6.3.1.

Now consider the bilinear form

$$
\mathscr{F} \times C_{0}^{\infty}(X) \ni(f, v) \rightarrow\langle f, v\rangle .
$$

For fixed $v$ it is continuous as a function of $f$. For fixed $f$ we can by (a) find $u \in \mathscr{D}^{\prime}(X)$ so that $P u=f$ and therefore $\langle f, v\rangle=\left\langle u,{ }^{t} P v\right\rangle$. Thus the bilinear form is continuous as a function of $v$ for fixed $f$ when supp ${ }^{t} P v \subset K$ for a fixed compact set $K$ and we put the $C^{\infty}$ topology on ${ }^{t} P v$. Since $\mathscr{F}$ is complete the separate continuity implies continuity, that is, for every compact set $K$ we find continuous semi-norms $N_{1}$ and $N_{2}$ in $C^{\infty}(X)$ such that

$$
|\langle f, v\rangle| \leqslant C N_{1}(f) N_{2}\left({ }^{t} P v\right) ; \quad f \in \mathscr{F}, v \in C_{0}^{\infty}(X), \operatorname{supp}^{t} P v \subset K .
$$

Choose a compact set $K^{\prime}$ so that $N_{1}(f)=0$ if $f=0$ near $K^{\prime}$. Then it follows that $\langle f, v\rangle=0$ if $v \in C_{0}^{\infty}(X), \operatorname{supp}^{t} P v \subset K, f \in \mathscr{F}$ and $f=0$ near $K^{\prime}$. Fix $v$ and set $K^{\prime \prime}=K^{\prime} U \operatorname{supp} v$. By a remark above $\langle f, v\rangle=0$ for all $f \in C_{0}^{\infty}(X)$ with $f=0$ in a neighborhood of $K^{\prime}$ and $f$ orthogonal to $N\left(K^{\prime \prime}\right)$. Since $N\left(K^{\prime \prime}\right)$ is finite dimensional it follows that for some $w \in N\left(K^{\prime \prime}\right)$

$$
\langle f, v\rangle=\langle t, w\rangle \quad \text { if } f \in C^{\infty}(X) \text { and } f=0 \text { in a neighborhood of } K^{\prime} .
$$

Hence supp $(v-w) \subset K^{\prime}$, and the proof is complete.

Theorem 6.3.4 does not really answer the question about global solvability for the conditions (c) and (d) remain to be analyzed. Some uniqueness theorems giving sufficient conditions may be found e.g. in Hörmander [17, Chap. VIII] in the case of differential operators. Further analysis lies outside the scope of this paper for we shall now give an 
example showing that hypotheses made on the operator $P$ modulo $C^{\infty}$ only cannot suffice to decide if $P$ has the properties discussed in Theorem 6.3.4. Our construction is based on the observation that for a hypoelliptic non-elliptic operator there are sets which are not $P$-convex (see Hörmander [17, section 3.7]). To be specific we consider the heat operator $Q(D)=D_{1}^{2}+i D_{2}$ in $\mathbf{R}^{2}$ and choose an open set $X \subset \mathbf{R}^{2}$ such that $\left\{x \in \mathbf{C} X ; x_{2} \geqslant 0\right\}$ contains 0 as an isolated point. Let $E(x-y)$ be a fundamental solution of $Q(D)$ which vanishes when $x_{2}<y_{2}$, and choose $F(x, y)$ also equal to 0 then so that $E(x-y)-F(x, y) \in C^{\infty}(X \times X)$ and $F$ is the kernel of a properly supported operator, also denoted by $F$. We now consider the properly supported operator

$$
P=Q(D) F
$$

By construction $P=I+R$ where $R$ has a $C^{\infty}$ kernel. Although $P$ differs from the identity only by an operator with $C^{\infty}$ kernel we shall show that the range of $P$ on $C^{\infty}(X)$ resp. $\mathscr{D}^{\prime}(X)$ is dense in $C^{\infty}(X)$ resp. $\mathscr{D}^{\prime}(X)$ but not equal to either space. To do so we first note that ${ }^{t} P v=0, v \in \mathscr{E}^{\prime}(X)$, implies $v=0$. In fact, $v=-{ }^{t} R v \in C_{0}^{\infty}(X)$ so we have

$$
v(x)=-\int R(y, x) v(y) d y .
$$

Let $V\left(x_{2}\right)=\int|v(x)| d x_{1}$ and choose $C$ so that

$$
\int_{x \in \operatorname{supp} v}|R(y, x)| d x_{1} \leqslant C, \quad y \in \operatorname{supp} v
$$

Then we have

$$
V\left(x_{2}\right) \leqslant C \int_{y_{2}>x_{3}} V\left(y_{2}\right) d y_{2},
$$

and since $V=0$ for large $x_{2}$ this implies that $V=0$ identically. Hence $v=0$. Since ${ }^{t} P v={ }^{t} F^{t} Q v$ we can easily show that (d) in Theorem 6.3.4 is not fulfilled by taking for $v$ a null solution with respect to a half plane $x_{2} \geqslant \varepsilon \rightarrow 0$, cut off to have compact support in $X$ by a fixed cutoff function. Since this is just a repetition of the proof of Theorem 3.7.1 in Hörmander [17] we leave the details for the reader.

When $P$ is a differential operator with constant coefficients the results of Andersson [29] concerning analytic singularities show that the hypotheses of Theorem 6.3.3 imply that $P \mathscr{D}^{\prime}(X)=\mathscr{D}^{\prime}(X)$. An extension to real analytic coefficients has been given by KawaiKashiwara (to be published) and Hörmander [44] under the almost certainly superfluous additional hypothesis that the bicharacteristics are regular, i.e., $\partial p / \partial \xi \neq 0$ when $p=0$.

In section 6.5 we shall construct parametrices for $P$ with properties which immediately give the implications (c) $\Rightarrow$ (a) and (c) $\Rightarrow$ (b) in Theorem 6.3.3. (See Theorem 6.5.10.) To do so we need some preliminary results on first order differential operators which will be proved in section 6.4 . 


\subsection{First order real differential operators}

Let $M$ be a $C^{\infty}$ manifold and $L$ a real $C^{\infty}$ vector field on $M$. We shall study the existence of solutions of the equation

$$
L u=f
$$

when $u, f \in C^{\infty}(M)$. If $K$ is a compact subset we denote as before by $C^{\infty}(K)$ the quotient of $C^{\infty}(M)$ by the subspace consisting of elements vanishing of infinite order on $K$. The dual space is then $\mathscr{E}^{\prime}(K)$.

THEOREM 6.4.1. Let $K$ be a compact subset of M. Then the following conditions are equivalent:

(a) $L C^{\infty}(K)=C^{\infty}(K)$.

(b) $(L+a) C^{\infty}(K)=C^{\infty}(K)$ for every $a \in C^{\infty}(K)$.

(c) There exists a function $\varphi \in C^{\infty}$ such that $L^{2} \varphi>0$ on $K$.

(d) No complete integral curve of $L$ is contained in $K$.

Proof. (a) $\Rightarrow$ (b) for if $L v=a$ and $L w=e^{v} f$ then $(L+a)\left(w e^{-v}\right)=f$. That (b) $\Rightarrow(\mathrm{a})$ is evident. Using (a) twice we find a function $\varphi \in C^{\infty}(X)$ with $L^{2} \varphi=1$ which proves (c). From (c) we obtain (d) by noting that if an integral curve $\Gamma$ is contained in $K$ and the maximum of $\varphi$ in $\bar{\Gamma}$ is attained at $y$, then the integral curve through $y$ is contained in $\bar{\Gamma}$ and $L \varphi(y)=$ $0, L^{2} \varphi(y)>0$. This is a contradiction. Finally, to prove that (d) $\Rightarrow$ (a) we first note that (d) implies

(d') No integral curve of $L$ is contained in $K$ for all positive or all negative values of the parameter.

In fact, the solution curve starting at a limit point of a half integral curve with this property would be entirely contained in $K$ in contradiction with (d). (This argument already occurred in the proof of Theorem 6.3.3.) In view of $\left(\mathrm{d}^{\prime}\right)$ every point $y \in K$ lies on an interval of an integral curve with end points outside $K$ so if $f \in C^{\infty}(M)$ has support sufficiently close to $y$ it is clear that the equation $L u=f$ can be solved in a neighborhood of $K$. By a partition of unity we conclude that this is also true for an arbitrary $f \in C^{\infty}(M)$. The proof is complete.

Theorem 6.4.1 is of course analogous to Theorem 6.3.1 which contains a less elementary proof that $(d) \Rightarrow(a)$. We now give an analogue of Theorem 6.3.3.

THEOREM 6.4.2. The following conditions (a)-(f) on L are equivalent:

(a) $L C^{\infty}(M)=C^{\infty}(M)$.

(b) $(L+a) C^{\infty}(M)=C^{\infty}(M)$ for every a $\in C^{\infty}(M)$. 
(c) There exists a function $\varphi \in C^{\infty}(M)$ such that $L^{2} \varphi>0$ and

is compact for every $c$.

$$
\{y \in M ; \varphi(y) \leqslant c\}
$$

(d) (1) No complete integral curve of $L$ is contained in a compact subset of $M$,

(2) for every compact subset $K$ of $M$ there exists a compact subset $K^{\prime}$ of $M$ such that every compact interval on an integral curve with end points in $K$ is contained in $K^{\prime}$.

(e) There are no periodic integral curves and the relation $R=\left\{\left(y_{1}, y_{2}\right) \in M \times M\right.$; $y_{1}$ and $y_{2}$ are on the same integral curve of $\left.L\right\}$ is a closed $C^{\infty}$ submanifold of $M \times M$.

(f) There exists a manifold $M_{0}$, an open neighborhood $M_{1}$ of $M_{0} \times\{0\}$ in $M_{0} \times \mathbf{R}$ which is convex in the $\mathbf{R}$ direction, and a diffeomorphism $M \rightarrow M_{1}$ which carries $L$ into the operator $\partial / \partial t$ if points in $M_{0} \times \mathbf{R}$ are denoted by $\left(y_{0}, t\right)$.

Proof. (a) $\Rightarrow$ (b) is obvious as in the proof of Theorem 6.4.1. That result also shows that $(\mathrm{a}) \Rightarrow(\mathrm{d} 1)$. To prove that $(\mathrm{a}) \Rightarrow(\mathrm{d} 2)$ we assume that $(\mathrm{d} 2)$ is not true. Then there exist intervals $\left[y_{j}^{\prime}, y_{j}^{\prime \prime}\right]$ on solution curves and $y_{j} \in\left[y_{j}^{\prime}, y_{j}^{\prime \prime}\right]$ such that $y_{j}^{\prime}, y_{j}^{\prime \prime} \in K$ but $y_{j}$ is not contained in any fixed compact set for all $j$. Taking a subsequence we may assume that $y_{j}^{\prime} \rightarrow y^{\prime}, y_{j}^{\prime \prime} \rightarrow y^{\prime \prime}$ and that any compact set contains only finitely many $y_{j}$. We can now take a $C^{\infty}$ non-negative function $f$ on $M$ which is so large near the points $y_{j}$ that $u\left(y_{j}^{\prime}\right)-u\left(y_{j}^{\prime \prime}\right)$, being the integral of $f$ over the integral curve, tends to $\infty$ with $j$ if $L u=f$. Thus (a) cannot be valid. By Theorem 6.4.1 we also have $(c) \Rightarrow(d l)$, and that $(c) \Rightarrow(d 2)$ is obvious.

(d) $\Rightarrow(\mathrm{e})$. Denote the $L$-flow by $\varphi$ so that $t \rightarrow \varphi(y, t)$ is the solution of the equation $d z / d t=L(z)$ with $z(0)=y$ defined on a maximal open interval $\subset \mathbf{R}$. If $D_{\varphi}$ is the domain of $\varphi$, then

$$
R=\left\{(\varphi(x, t), x) ;(x, t) \in D_{\varphi}\right\} .
$$

The map $(x, t) \rightarrow(\varphi(x, t), x)$ is injective since there are no closed bicharacteristic strips, and it is clear that the differential is also injective. To prove that $R$ is a closed $C^{\infty}$ submanifold it suffices therefore to show that the map is proper. Let $\left(x_{i}, t_{i}\right) \in D_{\varphi}$ and assume that $x_{i} \rightarrow x, \varphi\left(x_{i}, t_{i}\right) \rightarrow y$ in $M$. We have to show that $\left(x_{i}, t_{i}\right)$ has a limit point in $D_{\varphi}$. In doing so we may assume that $t_{i} \rightarrow T \in[-\infty, \infty]$. By (d2) there is a compact set $K^{\prime}$ such that $\varphi\left(x_{i}, t\right) \in K^{\prime}$ when $t \in\left[0, t_{i}\right]$. If $T= \pm \infty$ it follows that $\varphi(x, s) \in K^{\prime}$ for $s \geqslant 0$ or for $s \leqslant 0$. In view of the equivalence of $(d)$ and $\left(d^{\prime}\right)$ in Theorem 6.4.1 this contradicts (d l). Hence $T$ is finite and $\left(x_{i}, t_{i}\right) \rightarrow(x, T) \in D_{\varphi}$.

(e) $\Rightarrow$ (f). It follows from (e) that the quotient space $M_{0}=M / R$ is a Hausdorff space, and identifying a neighborhood of the equivalence class of $x$ with a manifold transversal to $L$ at $x$ we obtain a structure of $C^{\infty}$ manifold in $M_{0}$. The map $M \rightarrow M_{0}$ has a $C^{\infty}$ cross 
section $M_{0} \rightarrow M$. This is obvious locally and using a partition of unity in $M_{0}$ we can piece local sections together to a global one, for only an affine structure is required to form averages. We can now take $M_{1}=\left\{(x, t) ; x \in M_{0},(x, t) \in D_{\varphi}\right\}$ and the map $M_{1} \rightarrow M$ given by $\varphi$. (See also Steenrod [50], sections 12.2 and 6.7.)

(f) $\Rightarrow$ (a) for the equation $\partial u / \partial t=f \in C^{\infty}\left(M_{1}\right)$ has a unique solution $u \in C^{\infty}\left(M_{1}\right)$ with $u=0$ for $t=0$.

(f) $\Rightarrow(c)$. If $\varphi_{0}$ and $\varphi_{1}$ are positive $C^{\infty}$ functions in $M_{0}$ and in $M_{1}$, then

$$
\varphi\left(y_{0}, t\right)=\varphi_{0}\left(y_{0}\right)+\int_{0}^{t}(t-s) \varphi_{1}\left(y_{0}, s\right) d s
$$

is in $C^{\infty}\left(M_{1}\right)$ and $L^{2} \varphi>0$. If $\varphi_{0} \rightarrow \infty$ at $\infty$ in $M_{0}$ and $\varphi_{1} \rightarrow \infty$ sufficiently rapidly at $\infty$ in $M_{1}$, it follows that $\varphi \rightarrow \infty$ at $\infty$ in $M_{1}$.

Remark. The equivalence of (a), (b) and (d) is of course essentially contained in Malgrange [48]. According to Whitney [52] the $L$-flow is called parallelizable when (f) is fulfilled. For conditions equivalent to (f) see also Birkhoff [30, Chap. VII] and in the topological case Dugundji-Antosiewicz [36].

In the applications of Theorem 5.3.2 in section 6.5 we must solve equations of the form (6.4.1) when $M$ is a cone manifold (section 1.1, p. 87) and $u, f$ are symbols on $M$. We assume that the vector field $L$ commutes with multiplication by positive scalars as is the case for the Hamilton field of a function which is homogeneous of degree 1 . Thus $L u$ is homogeneous of degree $m$ if $u$ is, and $L u \in S_{e}^{m+1-e}(M)$ if $u \in S_{e}^{m}$. In particular, if $M_{s}$ is the quotient of $M$ by the action of $\mathbf{R}_{+}, L$ induces a vector field $L_{s}$ on $M_{s}$ since the $C^{\infty}$ functions on $M_{s}$ are precisely the $C^{\infty}$ functions on $M$ which are homogeneous of degree 0 . We write $\pi$ for the projection $M \rightarrow M_{s}$.

THEOR $\mathrm{M}$ 6.4.3. When $M$ is a cone manifold and $L$ a $C^{\infty}$ vector field commuting with multiplication by positive scalars in $M$, the following conditions are equivalent:

(i) For every $f \in S_{\varrho}^{m}(M), m \in \mathbf{R}, \frac{1}{2}<\varrho \leqslant 1$, the equation $L u=f$ has a solution $u \in S_{Q}^{m}(M)$.

(ii) The vector field $L_{s}$ on $M_{s}$ satisfies one of the equivalent conditions in Theorem 6.4.2.

(iii). The vector field $L$ on $M$ satisfies one of the equivalent conditions in Theorem 6.4.2, and if $N$ is a positive $C^{\infty}$ function on $M$ which is homogeneous of degree 1 , then for any compact set $K \subset M_{s}$

$$
N(y) \leqslant C_{E} N(z)
$$

if $\pi y, \pi z \in K$ and $y, z$ are on the same orbit of $L$.

(iv) There exists a $C^{\infty}$ manifold $M_{0}$, an open neighborhood $M^{\prime}$ of $M_{0} \times 0$ in $M_{0} \times \mathbf{R}$ which is convex in the direction of $\mathbf{R}$, and a diffeomorphism $M \rightarrow M^{\prime} \times \mathbf{R}_{+}$commuting with 
multiplication by posifive scalars (defined as identity in $M^{\prime}$ and standard multiplication in $\mathbf{R}_{+}$) such that $L$ is mapped to the vector field $\partial / \partial t$ if the variables in $M_{0} \times \mathbf{R} \times \mathbf{R}_{+}$are denoted by $\left(y_{0}, t, r\right)$.

Proof. (i) $\Rightarrow$ (ii). Let $f \in C^{\infty}\left(M_{s}\right)$ and consider $f$ as a homogeneous function of degree 0 on $M$. Choose a solution $u \in S_{Q}^{0}(M)$ of the equation $L u=f$. If $K$ is a compact subset of $M$ and if $K_{1}=\{t y ; t \geqslant 1, y \in K\}, K_{2}=\{t y ; t>0, y \in K\}$, then there is a constant $C$ such that $|u(y)|<C, y \in K_{1}$, and we claim that

$$
|u(y)-u(z)|<2 C, \quad \text { if } y, z \in K_{\mathbf{2}} \text { are on the same orbit. }
$$

In fact, if $t \in \mathbf{R}_{+}$and we write $t^{*} u(y)=u(t y), y \in M, t>0$, then

$$
L\left(t^{*} u-u\right)=t^{*} L u-L u=t^{*} f-f=0
$$

so $t^{*} u-u$ is constant on the orbits of $L$. Hence $u(t z)-u(z)=u(t y)-u(y)$, so

$$
|u(y)-u(z)|=|u(t y)-u(t z)| .
$$

For large $t$ we have $t y, t z \in K_{1}$ and (6.4.3) follows. Since $\pi K_{2}=\pi K$ may be any compact set in $M_{s}$ conditions (d1) and (d2) of Theorem 6.4.2 for $L_{s}$ follow immediately, already if we take $f=1$ in the case of $(\mathrm{d} 1)$.

(ii) $\Rightarrow$ (iv). First note that there is a cross section $M_{s} \rightarrow M$. In fact, we can construct a positive $C^{\infty}$ function $N$ on $M$ which is homogeneous of degree 1 by means of a partition of unity on $M_{s}$. The section $M_{s} \rightarrow M$ is then uniquely determined if we require that $N(m)=1$ in the range. From condition (f) in Theorem 6.4.2 applied to the vector field $L_{s}$ on $M_{s}$ we now obtain a diffeomorphism $M \rightarrow M^{\prime} \times \mathbf{R}_{+}$with $M^{\prime}$ as in condition (iv), which transforms $L$ to a vector field of the form

$$
L_{1}=\partial / \partial t+a\left(y_{0}, t\right) r \partial / \partial r
$$

where $a \in C^{\infty}\left(M^{\prime}\right)$. Now solve the equation

$$
\partial b\left(y_{0}, t\right) / \partial t+a\left(y_{0}, t\right)=0
$$

with $b \in C^{\infty}\left(M^{\prime}\right)$ and introduce the function $N_{1}\left(y_{0}, t, r\right)=r \exp b\left(y_{0}, t\right)$ which is homogeneous of degree 1 and satisfies the equation $L_{1} N_{1}=0$. The composition of the map $M \rightarrow M^{\prime} \times \mathbf{R}_{+}$ and the map

$$
M^{\prime} \times \mathbf{R}_{+} \ni\left(y_{0}, t, r\right) \rightarrow\left(y_{0}, t, N_{1}\left(y_{0}, t, r\right)\right) \in M^{\prime} \times \mathbf{R}_{+}
$$

will then have the properties required in (iv).

The implications (iv) $\Rightarrow$ (i) and (iv) $\Rightarrow$ (iii) are trivial so it only remains to show that (iii) $\Rightarrow$ (ii). It follows from (6.4.2) that every integral curve of $L_{s}$ contained in a compact 
subset of $M_{s}$ can be lifted to an integral curve of $L$ contained in a compact subset of $M$. Hence (dl) of Theorem 6.4.2 must be valid for $L_{s}$. To prove (d2) we let $K$ be a compact set in $M_{s}$ and set

$$
K_{1}=\left\{y \in M ; \pi(m) \in K, C_{\bar{K}}^{-1} \leqslant N(y) \leqslant C_{K}\right\}
$$

If we lift an integral curve of $L_{s}$, with end points in $K$ to $M$ so that it starts at a point with $N(y)=1$, then the other end point will belong to $K_{1}$ too. Hence the whole integral curre belongs to a fixed compact set $K_{1}^{\prime}$ in $M$ which completes the proof of (ii) and of the theorem.

Remark 1. Under the hypotheses of Theorem 6.4.2 (or 6.4.3) the vector field $(L, 0)$ on $M \times M$ defines a vector field $L$ on the relation manifold $R$ which satisfies the same conditions. In fact, this is obvious from conditions (f) and (iv) respectively.

Remark 2. The obvious proof of the equivalence $(a) \Leftrightarrow(b)$ in Theorem 6.4.2 also gives that (i) in Theorem 6.4.3 is equivalent to

(i') For every $f \in S_{\varrho}^{m}(M), m \in \mathbf{R}, \frac{1}{2}<\varrho \leqslant 1$, and $c \in S_{\varrho}^{0}(M)$ the equation $(L+c) u=f$ has a solution $u \in S_{e}^{m}(M)$.

Moreover, the solution can be prescribed arbitrarily within the class $S_{e}^{m}\left(M_{0} \times \mathbf{R}_{+}\right)$ on $M_{0} \times 0 \times \mathbf{R}_{+} \hookrightarrow M$, the injection being as in condition (iv) of Theorem 6.4.3.

Remark 3. The situation changes drastically if for example periodic integral curves occur. Assume that $d y(t) / d t=L(y(t))$ and that $y(t)$ is periodic with period $T$. The equation $L u+c u=f$ reduces to $d u / d t+c(y(t)) u=f(y(t))$ on this curve. Because $t \rightarrow u(y(t))$ has to be periodic, this leads if $d h(t) / d t=c(y(t)), h(0)=0$, to

$$
u(y(0)) \exp h(T)=u(y(0))+\int_{0}^{T} f(y(t)) \exp h(t) d t .
$$

It follows that the equation $L u+c u=f$ is solvable if and only if $\exp h(T) \neq 1$, that is,

$$
\int_{0}^{T} c(y(t)) d t \neq 2 k \pi i, k \in \mathbf{Z}
$$

Secondly, if this condition is satisfied then the solution $u$ is uniquely determined by $f$.

Now suppose that all integral curves of $L$ are periodic with a positive minimal period depending continuously on the initial point. This means that the relation $R$ introduced in Theorem 6.4.2 is a closed submanifold of $M \times M$, that the orbit space $M / R$ is a manifold and that $M \rightarrow M / R$ is a fibration with fibers diffeomorphic to the circle. Then the equation $L u+c u=f$ is globally solvable for every $f$ if and only if (6.4.4) is valid for each 
integral curve $y(t)$ of $L$, with $T$ denoting the period of the curve. This will be called the non-resonance case. Moreover, in this case the solution $u$ is uniquely determined by $f$. Analogous statements are of course valid in the case of cone manifolds.

\subsection{Construction of global parametrices}

A continuous operator $E: C_{0}^{\infty}(X) \rightarrow C^{\infty}(X)$ is called a right parametrix of the properly supported pseudo-differential operator $P$ if

$$
P E=I+R
$$

where $I$ is the identity and $R$ has a $C^{\infty}$ kernel. If instead $E P=I+R^{\prime}$ with $R^{\prime} \in C^{\infty}$ one calls $E$ a left parametrix. We shall say that $R$ is a parametrix if $E$ is both a right and a left parametrix.

Throughout this section we shall assume that $P$ is of real principal type in the manifold $X$ (Definition 6.3.2) and that $P$ satisfies condition (c) in Theorem 6.3.3. For easy reference we introduce a term for this property:

Definition 6.5.1. If $P$ is of real principal type in $X$ we shall say that $X$ is pseudoconvex with respect to $P$ when condition (c) in Theorem 6.3.3 is fulfilled.

Denote by $N$ the set of zeros of $p$ in $T^{*}(X) \backslash 0$. This is a conic manifold and the Hamilton field $H_{p}$ is tangential to $N$. The integral curves are the bicharacteristic strips of $P$ and we define the bicharacteristic relation $C$ by

$C=\{((x, \xi),(y, \eta)) \in N \times N ;(x, \xi)$ and $(y, \eta)$ lie on the same bicharacteristic strip $\}$.

This construction is invariant under the action of canonical transformations on $p$ since the definition of the Hamilton field is. Multiplication of $p$ by a non-vanishing function will change the parameter on the bicharacteristic strips but not the bicharacteristic relation. Note that the set $C_{n}$ defined by (6.1.2) is the bicharacteristic relation of $D_{n}$.

By the preceding remarks we may assume that $P$ is of degree 1 when studying $C$. By hypothesis the vector field $H_{p}$ on $N$ then satisfies condition (ii) of Theorem 6.4 .3 (in the form of condition (d) in Theorem 6.4.2) so the equivalent condition (iii) in Theorem 6.4.3 shows that $C$ is a closed conic submanifold of $\left(T^{*}(X) \backslash 0\right) \times\left(T^{*}(X) \backslash 0\right)$ which is closed in $T^{*}(X \times X) \backslash 0$ by (6.4.2). Conversely, if $P$ is of real principal type in $X$ and $C$ has these properties it follows that $X$ is pseudo-convex with respect to $P$.

We shall now prove that $C$ is a homogeneous canonical relation (Definition 4.1.2). To prove this we consider the flow $\varphi_{1}$, with domain $D$, defined by the Hamilton field $H_{p_{1}}$ of the function $p_{1}$ obtained by lifting $p$ from $T^{*}(X) \backslash 0$ to $\left(T^{*}(X) \backslash 0\right) \times\left(T^{*}(X) \backslash 0\right)$ by the projection on the first factor. $C$ is then the "flow-out" 


$$
\left\{\varphi_{1}(h, t) ;(h, t) \in D, h \in \Delta_{N}\right\}
$$

of the diagonal $\Delta_{N}$ in $N$. The symplectic form $\sigma_{1}-\sigma_{2}$ in $T^{*}(X) \times T^{*}(X)$, which is the difference of the symplectic form in each of the two copies, vanishes on $\Delta_{N}$, and $H_{p_{1}}$ is orthogonal to but not a member of $T\left(\Delta_{N}\right)$ since $p_{1}=0$ on $\Delta_{N}$ and $H_{p_{1}} \neq 0$. This proves that $C$ is Lagrangean at $\Delta_{N}$. Since $C$ is invariant under the Hamilton flow defined by $p_{1}$ and $\varphi_{1}(\cdot, t)$ is canonical with respect to $\sigma_{1}-\sigma_{2}$, it follows that $C$ is Lagrangean everywhere. Thus we have proved

Proposition 6.5.2. Assume that $P$ is of real principal type in $X$. Then the bicharacteristic relation $C$ of $\boldsymbol{P}$ is a homogeneous canonical relation from $T^{*}(X) \backslash 0$ to $T^{*}(X) \backslash 0$ if and only if $X$ is pseudo-convex.

$C \backslash \Delta_{N}$ is the disjoint union $C^{+} \cup C^{-}$of the forward (backward) bicharacteristic relations $C^{+}$and $C^{-}$defined as the set of all $((x, \xi),(y, \eta)) \in N \times N$ such that $(x, \xi)$ lies after (resp. before) $(y, \eta)$ on a bicharacteristic strip. These are open subsets of $C$ and inverse relations. The definition is invariant under multiplication of $p$ by positive functions but $C^{+}$and $C^{-}$are interchanged if we multiply by a negative function. The role of these sets is indicated by Proposition 6.1.2.

More generally, by an orientation of $C$ we shall mean any splitting of $C \backslash \Delta_{N}$ in a disjoint union of open subsets $C^{2}$ and $C^{2}$ which are inverse relations. In order to describe these, denote by $B(x, \xi)$ the bicharacteristic strip through $(x, \xi)$ and write

$$
C^{ \pm}(x, \xi)=C^{ \pm} \cap(B(x, \xi) \times B(x, \xi))
$$

Because $C^{j}$ is a union of components of $C \backslash \Delta_{N}$ we have either $C^{+}(x, \xi) \subset C^{j}$ or else $C^{+}(x, \xi) \cap C^{j}=\varnothing, j=1,2$. Write

$$
N^{j}=\left\{(x, \xi) \in N ; C^{+}(x, \xi) \subset C^{j}\right\}, j=1,2 .
$$

Then $N^{1}$ and $N^{2}$ are open, disjoint, and $N=N^{1} \cup N^{2}$. Conversely

$$
C^{1}=\left(\bigcup_{N^{2}} C^{+}(x, \xi)\right) \cup\left(\bigcup_{N^{2}} C^{-}(x, \xi)\right)
$$

and $C^{2}$ is obtained similarly by interchanging 1 and 2 (or + and - ). We can therefore identify the orientations of $C$ with the open and closed subsets of $N$. Denote by $\tilde{N}$ the set of all components of $N$, let $v$ be a subset of $\tilde{N}$. Denote by $N_{v}^{+}$, resp. $N_{v}^{-}$the union of the components in $v$, resp. in $\bar{N} \backslash \nu$ and write $C \backslash \Delta_{N}=C_{v}^{+} \cup C_{v}^{-}$for the orientation of $C$ corresponding as above to $N^{1}=N_{v}^{+}$and $N^{2}=N_{v}^{-}$. Observe that $C_{v}^{-}=C_{\tilde{N} \backslash v}^{+}$and that $C^{+}=C_{\tilde{N}}^{+}$. If $N$ has $k$ components there are $2^{k}$ possible orientations. In particular, if $X$ is connected and $P$ is a strictly hyperbolic differential operator of order $m$ then there are $2^{m}$ orientations. 
THЕОRем 6.5.3. Let $P$ be of real principal type in $X$ and assume that $X$ is bicharacteristically convex. For every orientation $C \backslash \Delta_{N}=C_{v}^{+} \cup C_{v}^{-}$one can then find parametrices $E_{p}^{+}$and $E_{v}^{-}$of $P$ with

$$
W F^{\prime}\left(E_{\nu}^{+}\right)=\Delta^{*} \cup C_{\nu}^{+}, \quad W F^{\prime}\left(E_{\nu}^{-}\right)=\Delta^{*} \cup C_{\nu}^{-}
$$

where $\Delta^{*}$ is the diagonal in $\left(T^{*}(X) \backslash 0\right) \times\left(T^{*}(X) \backslash 0\right)$. Any right or left parametrix $E$ with $W F^{\prime}(E)$ contained in $\Delta^{*} \cup C_{v}^{+}$resp. $\Delta^{*} \cup C_{v}^{-}$must be equal to $E_{v}^{+}$resp. $E_{\nu}^{-}$modulo $C^{\infty}$. For every $s \in \mathbf{R}$ the parametrices $E_{v}^{+}, E_{v}^{-}$define continuous maps from $H_{(s)}^{\text {comp }}(X)$ to $H_{(s+m-1)}(X)$. Finally

$$
E_{v}^{+}-E_{v}^{-} \in I_{1}^{\ddagger-m}\left(X \times X, C^{\prime}\right),
$$

and $E_{\nu}^{+}-E_{\nu}^{-}$is non-characteristic at every point of $C^{\prime}$.

Proof. We begin by proving the uniqueness. Assume that $E_{1}$ and $E_{2}$ are right and left parametrices with $W F^{\prime}\left(E_{j}\right) \subset \Delta^{*} \cup C_{\nu}^{+}$. We shall then prove that $E_{1}-E_{2} \in C^{\infty}$ by modifying an argument used in the proof of Proposition 2.5.1. This consisted in observing that $E_{2} P E_{1}$ is congruent both to $E_{1}$ and $E_{2} \bmod C^{\infty}$, but the definition of this product may be in doubt when $E_{1}$ and $E_{2}$ are not properly supported. However, we do know that $E_{2} B E_{1}$ is defined if $B$ is a pseudo-differential operator with kernel of compact support in $X \times X$, for $B$ maps $\mathscr{D}^{\prime}(X)$ to $\mathscr{E}^{\prime}(X)$ then. If $(x, \xi, y, \eta) \in W F^{\prime}\left(E_{2} B E_{1}\right)$ but $(x, \xi)$ and $(y, \eta)$ are both in the complement of $W F(B)$ it follows that $(x, \xi, z, \zeta) \in C_{\nu}^{+}$and that $(z, \zeta, y, \eta) \in C_{v}^{+}$for some $(z, \zeta) \in W F(B)$. This implies that $(x, \xi),(y, \eta),(z, \zeta)$ are on the same bicharacteristic strip with $(z, \zeta)$ between the other points. Let $K$ and $K^{\prime}$ be as in condition (c) of Theorem 6.3.3. If $W F(B)$ has no point over $K^{\prime}$ it follows that $W F^{\prime}\left(E_{2} B E_{1}\right)$ has no point in $K \times K$. Now choose $\varphi \in C_{0}^{\infty}(X)$ equal to 1 near $K^{\prime}$ and form

$$
E_{2} \varphi P E_{1}-E_{2} P \varphi E_{1}=E_{2}(\varphi P-P \varphi) E_{1}
$$

The wave front set of the right hand side contains no point over $K \times K$, so the same is true of $E_{2} \varphi-\varphi E_{1}$. Since $K$ is arbitrary it follows that $E_{2}-E_{1} \in C^{\infty}$.

Since $P E=I+R$ is equivalent to $E^{*} P^{*}=I+R^{*}$ and $P^{*}$ has the same principal symbol as $P$ the existence of left parametrices with the properties listed in the theorem follows from the existence of right parametrices for $P^{*}$. To prove the theorem it is therefore sufficient to construct a right parametrix with the required regularity properties. In doing so we may assume that the order of $P$ is 1 for $P$ can otherwise be replaced by the product with an elliptic operator of degree $1-m$ with positive homogeneous principal symbol. This has a pseudo-differential parametrix of degree $m-1$.

The first step in the construction is local in the cotangent bundle near the diagonal. 
LEMMA 6.5.4. Let $P \in L_{1}^{1}(X)$ satisfy the hypotheses of Theorem 6.5.3 and let $\left(x_{0}, \xi_{0}\right) \in$ $T^{*}(X) \backslash 0, p\left(x_{0}, \xi_{0}\right)=0$. If $T \in L_{1}^{0}(X)$ and $W F(T)$ is in a sufficiently small conic neighborhood of $\left(x_{0}, \xi_{0}\right)$, one can find $F^{+}, F^{-}$with $W F^{\prime}\left(F^{+}\right), W F^{\prime}\left(F^{-}\right)$close to $\left(x_{0}, \xi_{0}, x_{0}, \xi_{0}\right)$ such that $P F^{ \pm}=T+R^{ \pm}$and

(i) $W F^{\prime}\left(F^{ \pm}\right) \subset \Delta^{*} \cup C_{v}^{ \pm}$.

(ii) $R^{ \pm} \in I_{1}^{-\frac{1}{2}}\left(X \times X, C^{\prime}\right)$ and $W F^{\prime}\left(R^{ \pm}\right) \subset C_{v}^{ \pm}$.

(iii) $F^{+}-F^{-} \in I_{1}^{-\frac{1}{2}}\left(X \times X, C^{\prime}\right)$.

(iv) $F^{ \pm}$can be written in the form $A F_{n}^{ \pm} B$ where the kernel of $F_{n}^{ \pm}$is the product of the distribution $E_{n}^{ \pm}$in Proposition 6.1.2 by a $C^{\infty}$ function and $A, B$ are Fourier integral operators of order 0 belonging to inverse homogeneous local canonical transformations from $T^{*}(X) \backslash 0$ to $T^{*}\left(\mathbf{R}^{n}\right) \backslash 0$.

Proof. We may assume that $C_{\nu}^{+}=C^{+}$and $C_{\nu}^{-}=C^{-}$in a neighborhood of $\left(x_{0}, \xi_{0}\right)$ for in the opposite case we just have to consider $-P$ instead of $P$. Choose a canonical transformation $\chi$ and Fourier integral operators $A, B$ of degree 0 according to Proposition 6.1.4 and (6.1.13) with $\mu=0$. Then we have

$$
\chi^{-1} \circ C_{n}^{ \pm} \circ \chi \subset C^{ \pm}
$$

in view of the invariance of the definition of $C$ under canonical transformations. We shall prove that the composition

$$
F^{ \pm}=A F_{n}^{ \pm} B T
$$

has the required properties if $W F(T)$ is in a sufficiently small conic neighborhood of $\left(x_{0}, \xi_{0}\right)$ and $F_{n}^{ \pm}=\psi E_{n}^{ \pm}$where $\psi \in C^{\infty}\left(\mathbf{R}^{n} \times \mathbf{R}^{n}\right), \psi=1$ in a neighborhood of the diagonal and $\psi=0$ outside another sufficiently small neighborhood. Conditions (i) and (iii) in Lemma 6.5.4 follow immediately from (6.5.5), the corresponding conditions in Proposition 6.1.2, the calculus of Fourier integral operators developed in section 4.2, and Theorem 2.5.15. To prove (ii) we form

$$
P F^{ \pm}=P A F_{n}^{ \pm} B T=\left(P A-A D_{n}\right) F_{n}^{ \pm} B T+A D_{n} F_{n}^{ \pm} B T
$$

By (iii) in Proposition 6.1.4 we have

$$
\left(x_{0}, \xi_{0}, X_{0}, \Xi_{0}\right) \notin W F^{\prime}\left(P A-A D_{n}\right) \subset \Gamma \text {. }
$$

It follows that there is a conical neighborhood $V$ of $\left(X_{0}, \Xi_{0}\right)$ such that $\left(P A-A D_{n}\right) v \in C^{\infty}$ if $W F(v) \subset V$. Since $W F^{\prime}\left(F_{n}^{ \pm}\right)$can be made arbitrarily close to the diagonal in 
$\left(T^{*}\left(\mathbf{R}^{n}\right) \backslash 0\right) \times\left(T^{*}\left(\mathbf{R}^{n}\right) \backslash 0\right)$ by choosing the support of $\psi$ close to the diagonal in $\mathbf{R}^{n} \times \mathbf{R}^{n}$, we can choose $\psi$ and a conie neighborhood $V^{\prime}$ of $\left(X_{0}, \Xi_{0}\right)$ such that $W F\left(F_{n}^{ \pm} v\right) \subset V$, if $W F(v) \subset V^{\prime}$. If $W F(T)$ is so close to $\left(x_{0}, \xi_{0}\right)$ that $\chi W F^{\prime}(T) \subset V^{\prime}$ it follows that the first term on the right hand side of (6.5.6) is in $C^{\infty}$.

To study the last term in (6.5.6) we note that $D_{n} F_{n}^{ \pm}=I+R_{n}^{ \pm}$where

$$
R_{n}^{ \pm}=\left(D_{x_{n}} \psi(x, y)\right) E_{n}^{ \pm} \in I_{1}^{-\frac{1}{2}}\left(\mathbf{R}^{n} \times \mathbf{R}^{n}, C_{n}^{\prime}\right), \quad W F^{\prime}\left(R_{n}^{ \pm}\right) \subset C_{n}^{ \pm} .
$$

Since $A B T=(A B-I) T+T$ and $(A B-I) T \in C^{\infty}$ if $W F(T)$ is sufficiently close to $\left(x_{0}, \xi_{0}\right)$, it follows that $P F^{ \pm}=T+R^{ \pm}$where $R^{ \pm}-A R_{n}^{ \pm} B T \in C^{\infty}$. The calculus of Fourier integral operators now gives (ii).

End of proof of Theorem 6.5.3. If $\left(x_{0}, \xi_{0}\right) \in T^{*}(X) \backslash 0$ and $p\left(x_{0}, \xi_{0}\right) \neq 0$ a stronger result than Lemma 6.5.4 is valid: we can find a pseudo-differential operator $F$ such that $P F=T+R$ where $R \in C^{\infty}$ (see section 5.1). In this case as in Lemma 6.5.4 we can choose $W F^{\prime}(F)$ in any given neighborhood of $W F(T) \times W F(T)$, in fact as the diagonal in this product.

Let $\left\{V_{i}\right\}$ be a locally finite covering of $T^{*}(X) \backslash 0$ by open cones such that either Lemma 6.5.4 or the preceding observation is applicable when $W F(T) \subset V_{i}$. The projections $W_{i}$ of $V_{i}$ in $X$ are also locally finite. Using a partition of unity in the sphere bundle of $T^{*}(X) \backslash 0$ subordinate to the covering given by $\left\{V_{i}\right\}$ we can write $I=\sum T_{i}$ where $W F\left(T_{i}\right) \subset V_{i}$ and the support of the kernel of $T_{i}$ belongs to $W_{i} \times W_{i}$. For every $i$ we choose $F_{i}^{+}$according to Lemma 6.5.4 or as indicated above with supp $F_{i}^{ \pm} \subset W_{i} \times W_{i}$. Then the sum

$$
F^{ \pm}=\sum F_{i}^{ \pm}
$$

is defined; (6.5.3), (6.5.4) are satisfied by these operators and $F^{ \pm}$maps $H_{(s)}^{\text {comp }}(X)$ continuously into $H_{(s)}(X)$ by Theorem 4.3.1 because $E_{n}^{ \pm}$does. Further

$$
P F^{ \pm}=I+R^{ \pm} \quad \text { where } R^{ \pm} \in I_{1}^{-\frac{1}{2}}\left(X \times X, C^{\prime}\right), W F^{\prime}\left(R^{ \pm}\right) \subset C_{w}^{ \pm} .
$$

By Theorem 5.3.2 we can choose $G^{ \pm} \in I_{1}^{-\frac{1}{2}}\left(X \times X, C^{\prime}\right)$ so that

$$
P G^{ \pm}-R^{ \pm} \in C^{\infty} \text {. }
$$

In fact, the hypotheses of Theorem 5.3.2 are fulfilled in view of Remark 1 following Theorem 6.4.3 since the vector field $H_{p}$ satisfies the conditions in Theorem 6.4.3. If in the proof of Theorem 5.3.2 we choose initial data 0 on the diagonal of $N \times N$ for all the first order equations to be solved, we obtain $W F^{\prime}\left(G^{ \pm}\right) \subset C_{v}^{ \pm}$. It follows from Theorem 4.3.2 that $G^{ \pm}$is a continuous map from $H_{(s)}^{\text {comp }}(X)$ to $H_{(s)}(X)$ for every $s$, so $E_{p}^{ \pm}=F^{ \pm}-G^{ \pm}$is a right parametrix which is continuous from $H_{(s)}^{\text {comp }}(X)$ to $H_{(s)}(X)$. The construction shows 
that $F^{+}-F^{-}$and therefore $E_{p}^{+}-E_{v}^{-}$is non-characteristic at the diagonal of $N$. Since $P\left(E_{v}^{+}-E_{v}^{-}\right) \in C^{\infty}$ it follows from Theorem 5.3.1 that the principal symbol satisfies a first order homogeneous differential equation along the bicharacteristic strips starting there. Hence $E_{v}^{+}-E_{v}^{-}$is non-characteristic everywhere. (In section 6.6 we shall compute the principal symbol precisely.) This implies that $W F^{\prime \prime}\left(E_{v}^{+}-E_{v}^{-}\right)=C$ and since $W F^{\prime}\left(E_{v}^{ \pm}\right) \subset$ $\Delta^{*} \cup C_{v}^{ \pm}$we conclude that $W F^{\prime}\left(E_{\nu}^{ \pm}\right) \supset C_{\nu}^{ \pm}$. Since

$$
\Delta^{*}=W F^{\prime}(I)=W F^{\prime}\left(P E_{v}^{ \pm}\right) \subset W F^{\prime \prime}\left(E_{v}^{ \pm}\right)
$$

the proof of (6.5.3) is complete, and so is that of the theorem.

Remark 1. Suitably modified Lemma 6.5.4 remains valid under the weaker hypotheses of Proposition 6.1.4. If no open interval on a bicharacteristic strip has a fixed projection in $X$ the first part of the preceding argument gives a local parametrix.

Remark 2. If $p\left(x_{0}, \xi_{0}\right) \neq 0$ the proof shows that there is a pseudo-differential operator $Q$ such that $\left(x_{0}, \xi_{0}, x_{0}, \xi_{0}\right) \notin W F^{\prime}\left(E_{v}^{ \pm}-Q\right)$. The symbol of $Q$ near $\left(x_{0}, \xi_{0}\right)$ can be computed as in section 5.1 by the usual algorithm for determining the parametrix of an elliptic operator. Somewhat loosely we might say that $E_{v}^{ \pm}$is a pseudo-differential operator except at the diagonal of $N \times N, N=p^{-1}(0)$, where the symbol becomes highly singular and forces additional singularities on $C$. We shall now prove that these cannot be avoided.

At the end of the proof of Theorem 6.5.3 we saw that $W F^{\prime}(E) \supset \Delta^{*}$ for any right or left parametrix $E \in \mathscr{D}^{\prime}(X \times X)$ of $P$. We shall now prove that additional conditions on $W F^{\prime}(E)$ follow from a modification of Theorem 6.1.1.

LеммA 6.5.5. Let $P \in L_{1}^{m}(X)$ have a real and homogeneous principal part $p$, let $A \in \mathscr{D}^{\prime}(X \times X)$ and assume that $P A \in C^{\infty}(X \times X)$, where $A$ is considered as an operator $C_{0}^{\infty}(X) \rightarrow \mathscr{D}^{\prime}(X)$. If $(x, \xi, y, \eta) \in W F^{\prime}(A)$ and $\xi \neq 0$ it follows then that $p(x, \xi)=0$ and that $B(x, \xi) \times\{(y, \eta)\} \subset W \boldsymbol{F}^{\prime}(A)$.

Recall that $B(x, \xi)$ is the bicharacteristic strip containing $(x, \xi)$.

Proof. The hypothesis means that for some $F \in C^{\infty}(X \times X)$

$$
\left\langle A,{ }^{t} P u \otimes v\right\rangle=\langle F, u \otimes v\rangle ; \quad u, v \in C_{0}^{\infty}(X) .
$$

Thus

$$
\langle A, Q w\rangle=\langle F, w\rangle, \quad w \in C_{0}^{\infty}(X \times X),
$$

if $Q={ }^{t} P \otimes 1$ is defined by $Q w(x, y)={ }^{t} P w(x, y)$ with ${ }^{t} P$ acting on $w$ as a function of $x$ for fixed $y$. We cannot apply Theorem 6.1.1 directly since $Q$ is not a pseudo-differential operator. Choose a pseudo-differential operator $R$ of order 0 in $X \times X$ with principal part homo- 
geneous of degree 0 such that $W F(R)$ contains no element of the form $(x, 0, y, \eta)$. Thus $|\eta|<C|\xi|$ if $(x, \xi, y, \eta) \in W F(R)$ and $(x, y)$ is in a compact subset of $X \times X$. We can take the principal part of ${ }^{t} R$ equal to 1 on $B(x, \xi) \times\{(y, \eta)\}$. The proof of the multiplicative properties of pseudo-differential operators given in section 2.1 applies with no change to prove that $Q R=Q_{1}$ is a pseudo-differential operator with principal symbol given by the product of those of the two factors. If we replace $w$ by $R w$ in (6.5.7) it follows that

$$
{ }^{t} Q_{1} A={ }^{t} R F \in C^{\infty}
$$

so the lemma follows from Theorem 6.1.1 since $B \times\{(y, \eta)\}$ is a bicharacteristic strip of ${ }^{t} Q_{1}$.

We shall also need the analogous result where $A P \in C^{\infty}$ instead. This means that the operator $P \otimes 1$ above is replaced by $1 \otimes{ }^{t} P$. In terms of local coordinates, if $p_{x}^{\prime}=a, p_{\xi}^{\prime}=b$, the Hamilton equations corresponding to the principal symbol of ${ }^{t} P$ are

$$
d y / d t=-b(y,-\eta), \quad d \eta / d t=-a(y,-\eta)
$$

and this proves that $(y,-\eta)$ describes a bicharacteristic strip of $P$. If $A P \in C^{\infty}$ and $(x, \xi, y, \eta) \in W F^{\prime}(A), \eta \neq 0$, it follows therefore that $p(y, \eta)=0$ and

$$
W F^{\prime}(A) \supset\{(x, \xi)\} \times B(y, \eta) .
$$

Finally, if $P A \in C^{\infty}$ and $A P \in C^{\infty},(x, \xi, y, \eta) \in W F^{\prime}(A)$ and $\xi, \eta \neq 0$, it follows by repeated application of these results that $p(x, \xi)=p(y, \eta)=0$ and that

$$
W F^{\prime}(A) \supset B(x, \xi) \times B(y, \eta) .
$$

Remark. The hypothesis $P A \in C^{\infty}(X \times X)$ in Lemma 6.5.5 may of course by replaced by $W F^{\prime}(P A) \cap(B(x, \xi) \times\{(y, \eta)\})=\varnothing$.

In the following theorem we shall use the notation

$$
N=\left\{(x, \xi) \in T^{*}(X) \backslash 0, p(x, \xi)=0\right\} .
$$

When $(x, \xi) \in N$ we write

$$
\begin{aligned}
& C_{l}^{ \pm}(x, \xi)=C^{ \pm}(x, \xi) \cap(\{(x, \xi)\} \times N)=\{(x, \xi)\} \times B^{\mp}(x, \xi) \\
& C_{r}^{ \pm}(x, \xi)=C^{ \pm}(x, \xi) \cap(N \times\{(x, \xi)\})=B^{ \pm}(x, \xi) \times\{(x, \xi)\} .
\end{aligned}
$$

Here $C^{ \pm}(x, \xi)=C^{ \pm} \cap(B(x, \xi) \times B(x, \xi))$ as before and

$$
B^{ \pm}(x, \xi)=\left\{(y, \eta) \in B(x, \xi) ;(y, \eta, x, \xi) \in C^{ \pm}\right\} .
$$

THEOREM 6.5.6. If $E \in \mathscr{D}^{\prime}(X \times X)$ is either (a) a right parametrix, (b) a left parametrix or (c) a parametrix of an operator $P$ satisfying the hypotheses of Theorem 6.5.3, then $W F^{\prime}(E) \supset \Delta^{*}$ and respectively 
(a) For every $(x, \xi) \in N$ we have either $W F^{\prime}(E) \supset C_{r}^{+}(x, \xi)$ or $W F^{\prime}(E) \supset C_{r}^{-}(x, \xi)$.

(b) For every $(x, \xi) \in N$ we have either $W F^{\prime}(E) \supset C_{l}^{+}(x, \xi)$ or $W F^{\prime}(E) \supset C_{l}^{-}(x, \xi)$.

(c) For every $(x, \xi) \in N$ we have either $W F^{\prime}(E) \supset C^{+}(x, \xi)$ or $W F^{\prime}(E) \supset C^{-}(x, \xi)$.

Proof. That $W F^{\prime}(E) \supset \Delta^{*}$ follows from the end of the proof of Theorem 6.5.3. Let $E^{+}$and $E^{-}$be the parametrices given by Theorem 6.5.3 for the orientation $C^{+}, C^{-}$. We can apply Lemma 6.5.5 and the observations following it to $A=E-E^{+}$and $A=E-E^{-}$. Assume for example that $E$ is a right parametrix, that $(x, \xi) \in N$ and that $C_{r}^{+}(x, \xi)$ is not a subset of $W F^{\prime}(E)$. Since $C_{\uparrow}^{+}(x, \xi)$ does not meet $W F^{\prime}\left(E^{-}\right)$it follows that $C_{\uparrow}^{+}(x, \xi)$ is not a subset of $W F^{\prime}\left(E-E^{-}\right)$. Hence Lemma 6.5 .5 shows that $B(x, \xi) \times\{(x, \xi)\}$ does not meet $W F^{\prime}\left(E-E^{-}\right)$. In view of (6.5.3) this implies that $C_{r}^{-}(x, \xi) \subset W F^{\prime}(E)$. The other cases of the theorem are proved in exactly the same way.

The preceding arguments also allow us to supplement the uniqueness statement in Theorem 6.5.3.

THEOREM 6.5.7. Let $C_{\alpha}^{ \pm}$and $C_{\beta}^{ \pm}$be orientations of $C$ corresponding to two subsets $\alpha$ and $\beta$ of $\tilde{N}$. Denote by $N_{\alpha \beta}$ the symmetric difference of $N_{\alpha}^{+}$and $N_{\beta}^{+}$(or $N_{\alpha}^{-}$and $N_{\beta}^{-}$) consisting of points belonging to one set and not the other. If $E_{\alpha}^{+}$and $E_{\beta}^{+}$are the corresponding parametrices given by Theorem 6.5.3 then

$$
E_{\alpha}^{+}-E_{\beta}^{+} \in I_{1}^{\frac{1}{2}-m}\left(X \times X,\left(C \cap\left(N_{\alpha \beta} \times N_{\alpha \beta}\right)\right)^{\prime}\right) .
$$

In particular, $N_{\alpha \beta}$ is empty when the orientations are equal and $N_{\alpha \beta}=N$ when they are opposite, so $(6.5 .8)$ contains $(6.5 .4)$ and the uniqueness in Theorem 6.5.3.

Proof. If $(x, \xi) \in N$ then $W F^{\prime}\left(E_{\alpha}^{+}-E_{\beta}^{+}\right)$either contains $B(x, \xi) \times B(x, \xi)$ or else does not meet this set. Since $W F^{\prime}\left(E_{\gamma}^{+}\right) \cap(B(x, \xi) \times B(x, \xi))$ is one of the half spaces in $B \times B$, which one depending on whether $(x, \xi) \in N_{\gamma}^{+}$or $(x, \xi) \in N_{\gamma}^{-}$, it follows that

$$
W F^{\prime}\left(E_{\alpha}^{+}-E_{\beta}^{+}\right) \subset C \cap\left(N_{\alpha \beta} \times N_{\alpha \beta}\right)
$$

If $M$ is the complement of $N_{\alpha \beta}$ it follows by change of the orientation $\mathrm{C}_{\beta}^{ \pm}$that

$$
W F^{\prime}\left(E_{\alpha}^{+}-E_{\beta}^{-}\right) \subset C \cap(M \times M) .
$$

Since $E_{\alpha}^{+}-E_{\beta}^{+}=\left(E_{\alpha}^{+}-E_{\beta}^{-}\right)+\left(E_{\beta}^{-}-E_{\beta}^{+}\right)$we now obtain (6.5.8) from (6.5.4).

In section 6.6 we shall continue the study of the differences in (6.5.8). However we return now to the study of wave front sets of parametrices and shall prove a converse of Theorem 6.5.6. 
THEOREM 6.5.8. Let $F \subset\left(T^{*}(X) \backslash 0\right) \times\left(T^{*}(X) \backslash 0\right)$ be a closed conic set containing the diagonal and assume that either

(a) for every $(x, \xi) \in N$ we have $C_{r}^{+}(x, \xi) \subset F$ or $C_{r}^{-}(x, \xi) \subset F$;

(b) for every $(x, \xi) \in N$ we have $C_{l}^{+}(x, \xi) \subset F$ or $C_{l}^{-}(x, \xi) \subset F$;

(c) for every $(x, \xi) \in N$ we have $C^{+}(x, \xi) \subset F$ or $C^{-}(x, \xi) \subset F$.

Then there exists respectively (a) a right parametrix (b) a left parametrix (c) a parametrix $E$ such that

$$
W F^{\prime}(E) \subset F, \quad E H_{(s)}^{\text {comp }}(X) \subset H_{(s+m-1)}(X), \quad s \in \mathbf{R}
$$

If $\frac{1}{2}<\varrho<1$ one can choose $E$ so that in addition

$$
E-E_{v}^{ \pm} \in I_{\varrho}^{\frac{1}{2}-m}\left(X \times X, C^{\prime}\right)
$$

if $E_{v}^{ \pm}$is defined as in Theorem 6.5.3.

Proof. We may assume in the proof that $C_{v}^{+}=C^{+}$for Theorem 6.5.7 shows that (6.5.9) does not change if we change the orientation. In case (a) we denote by $\mathrm{N}^{+}$and $\mathrm{N}^{-}$the sets of all $(x, \xi) \in N$ with $C_{r}^{+}(x, \xi) \subset F$ resp. $C_{r}^{-}(x, \xi) \subset F$. An analogous definition is made in cases (b) and (c). Then $N^{+}$and $N^{-}$are closed conic sets with $N^{+} \cup N^{-}=N$. In case (c) we know in addition that $N^{+}$and $N^{-}$are closed under the equivalence relation $C$, that is, inverse images of sets in $N / C$. We shall write $E^{ \pm}=E_{\tilde{\tilde{N}}}^{ \pm}$.

If $A^{+}$and $A^{-} \in L_{\varrho}^{0}(X)$ are properly supported and if $A^{+}+A^{-}=I$, then $E=E^{+} A^{+}+E^{-} A^{-}$ is a right parametrix and $E=A^{+} E^{+}+A^{-} E^{-}$is a left parametrix. We have $E-E^{+}=$ $\left(E^{-}-E^{+}\right) A^{-}$and $E-E^{+}=A^{-}\left(E^{-}-E^{+}\right)$in the two cases. This gives (6.5.9) as a consequence of (6.5.4), and the continuity properties stated in the theorem follow from those in Theorem 6.5.3 and those of pseudo-differential operators. Since there exist closed conic subsets $\Gamma^{+}$and $\Gamma^{-}$of $T^{*}(X) \backslash 0$ with $\Gamma^{+} \cap N=N^{+}, \Gamma^{-} \cap N=N^{-}$and $\Gamma^{+} \cup \Gamma^{-}=T^{*}(X) \backslash 0$, the cases (a) and (b) in the lemma therefore follow from

LEM A 6.5.9. Let $\Gamma_{1}, \ldots, \Gamma_{j}$ be closed cones in $T^{*}(X) \backslash 0$ and let $\frac{1}{2}<\varrho<1$. Then one can find properly supported operators $A_{1}, \ldots, A_{j} \in L_{\varrho}^{0}(X)$ with $A_{1}+\ldots+A_{j}=I$ and $W F^{\prime \prime}\left(A_{i}\right) \subset \Gamma_{i}$, $i=1, \ldots, j$, if and only if

$$
\bigcup_{i=1}^{j} \Gamma_{i}=T^{*}(X) \backslash 0
$$

Proof. The necessity is trivial. In the proof of the sufficiency we may assume that $X \subset \mathbf{R}^{n}$. Let $\varphi_{i}(x, \xi, \varepsilon)$ be the convolution of the characteristic function of $\Gamma_{i}$ and $\varepsilon^{-2 n}$ $\chi(x / \varepsilon, \xi / \varepsilon)$ where $0 \leqslant \chi \in C_{0}^{\infty}, \chi=0$ outside the unit ball and $\int \chi d x d \xi=1$. Clearly the derivatives 
of $\varphi_{i}$ of order $k$ with respect to $x, \xi, \varepsilon$ can be estimated by $C_{k} \varepsilon^{-k}$, and $\Sigma \varphi_{i} \geqslant 1$. In view of this fact and Proposition 1.1.8 the functions defined by

$$
a_{i}(x, \xi)=\varphi_{i}(x, \xi /|\xi|, \varepsilon) / \sum_{1}^{j} \varphi_{k}(x, \xi /|\xi|, \varepsilon), \quad \varepsilon=|\xi|^{0-1},
$$

when $|\xi|>1$ and some smooth extension when $|\xi|<1$ are in $S_{0}^{0}\left(X \times \mathbf{R}^{n}\right)$. Furthermore, $a_{i}=0$ for large $|\xi|$ in the complement of any conic neighborhood of $\Gamma_{i}$ so $A_{i}=a_{i}(x, D)$ has the required properties.

Continued proof of Theorem 6.5.8. What remains to prove is part (c) which concerns the existence of a parametrix. Again we shall write $E=E^{+} A^{+}+E^{-} A^{-}$with $A^{+}+A^{-}=I$, which is a right parametrix. Since

$$
E-E^{+}=\left(E^{-}-E^{+}\right) A^{-}
$$

and

$$
\left(E^{-}-E^{+}\right) A^{-} P=\left(E^{-}-E^{+}\right) P A^{-}+\left(E^{-}-E^{+}\right)\left[A^{-}, P\right],
$$

we also have a left parametrix if

$$
W F\left(\left[A^{-}, P\right]\right) \cap N=\varnothing .
$$

(Since $\left[A^{-}, P\right]=-\left[A^{+}, P\right]$ the operators $A^{+}$and $A^{-}$play a symmetric role here.) If in addition to $(6.5 .10)$ we have

$$
W F\left(A^{+}\right) \cap N \subset N^{+}, W F^{\prime}\left(A^{-}\right) \cap N \subset N^{-},
$$

it follows that $W F^{\prime}(E) \subset F$. Using the fact that $N^{+}$and $N^{-}$are closed under the equivalence relation $C$ we shall construct such operators $A^{+}$and $A^{-}$in section 6.6. No reference to part (c) of Theorem 6.5.8 will be made until then.

We shall now apply the established part of Theorem 6.5.8 to give another more constructive proof of the implications $(c) \Rightarrow(a)$ and $(c) \Rightarrow(b)$ in Theorem 6.3.3. Extending a terminology introduced in section 2.1 we shall say that a distribution $K \in \mathscr{D}^{\prime}(X \times Y)$ is left (right) properly supported if the projection $\operatorname{supp} K \ni(x, y) \rightarrow x(\operatorname{resp}(x, y) \rightarrow y)$ is a proper map. The map $C_{0}^{\infty}(Y) \rightarrow \mathscr{D}^{\prime}(X)$ defined by $K$ (which we also denote by $K$ ) can then be extended to a map $C^{\infty}(Y) \rightarrow \mathscr{D}^{\prime}(X)$ (resp. is a map $C_{0}^{\infty}(Y) \rightarrow \mathscr{E}^{\prime}(X)$ ). If $W F_{Y}^{\prime}(K)=\varnothing$ the maps will be from $\mathscr{D}^{\prime}(Y)$ to $\mathscr{D}^{\prime}(X)$ and $\mathscr{E}^{\prime}(Y)$ to $\mathscr{E}^{\prime}(X)$ of course.

Тн ЕО Вем 6.5.10. Let $P$ be as in Theorem 6.5.3. Then one can find a left properly supported right parametrix $E_{1}$ and a right properly supported left parametrix $E_{2}$ for $P$ such that for $j=1,2$ and a given $\varrho$ with $\frac{1}{2}<\varrho<1$

(i) $W F^{\prime \prime}\left(E_{j}\right) \subset \Delta^{*} \cup C$,

(ii) $E_{j}-E_{v}^{ \pm} \in I_{0}^{\frac{1}{b}-m}\left(X \times X, C^{\prime}\right)$ if $E_{v}^{ \pm}$is defined as in Theorem 6.5.3,

(iii) $E_{j}$ is continuous from $H_{(s)}^{\text {comp }}(X)$ to $H_{(s+m-1)}(X)$ for every $s \in \mathbf{R}$. 
Proof. As in the proof of Theorem 6.5.3 the statement concerning the left parametrix follows from that about the right parametrix by passing to adjoints. It suffices therefore to show that $E_{1}$ can be chosen according to Theorem 6.5.8, part (a), so that sing supp $E_{1} \ni$ $(x, y) \rightarrow x \in X$ is a proper map. For then there is a neighborhood $V$ of $\operatorname{sing} \operatorname{supp} E_{1}$ such that $V \ni(x, y) \rightarrow x \in X$ is also proper. If $\chi \in C^{\infty}(X \times X)$ has support in $V$ and $\chi=1$ in a neighborhood of sing supp $E_{1}$, then $\chi E_{1}$ is a left properly supported right parametrix with the properties required in the theorem.

Let $X_{1}, X_{2}, \ldots$ be an increasing sequence of open relatively compact subsets of $X$ with union $X$ and choose another such sequence $X_{j}^{\prime}$ such that any interval on a bicharacteristic curve with end points in $X_{j}$ belongs to $X_{j}^{\prime}$. That this is possible follows of course from the pseudo-convexity of $X$. We shall apply Theorem 6.5 .8 with

$$
F^{\prime}=\Delta^{*} \cup\left(\bigcup_{N^{+}} C_{r}^{+}(x, \xi)\right) \cup\left(\bigcup_{N^{-}} C_{r}^{-}(x, \xi)\right)
$$

where with $\pi$ denoting the projection $T^{*}(X) \rightarrow X$

$$
\begin{array}{ll}
N^{+}=\left\{(x, \xi) \in N ; \pi B^{+}(x, \xi) \subset \mathbf{C} X_{j}\right. & \text { for every } \left.j \text { with } x \notin X_{j}^{\prime}\right\} \\
N^{-}=\left\{(x, \xi) \in N ; \pi B^{-}(x, \xi) \subset \mathbf{C} X_{j} \quad \text { for every } j \text { with } x \notin X_{j}^{\prime}\right\} .
\end{array}
$$

These are closed cones and $N^{+} \cup N^{-}=N$. In fact, if $(x, \xi)$ is not in $N^{+} \cup N^{-}$but $(x, \xi) \in N$ and if $j$ is the largest integer with $x \notin X_{j}^{\prime}$ then $B(x, \xi)$ contains points over $X_{j}$ on either side of $(x, \xi)$ in contradiction with the definition of $X_{j}^{\prime}$. Thus $F$ satisfies the hypotheses of Theorem 6.5.8. If $(x, \xi, y, \eta) \in F^{\prime}$ and $x \in X_{j}$, then the definition of $F$ gives $y \in X_{j}^{\prime}$ so the theorem is proved.

Theorem 6.5.8 shows that the minimal sets $F \subset\left(T^{*}(X) \backslash 0\right) \times\left(T^{*}(X) \backslash 0\right)$ containing $W F^{\prime}(E)$ for some (a) right parametrix (b) left parametrix or (c) parametrix are precisely all sets of the form

$$
\begin{aligned}
& F=\Delta^{*} \cup\left(\bigcup_{N^{+}} C_{r}^{+}(x, \xi)\right) \cup\left(\bigcup_{N^{-}} C_{r}^{-}(x, \xi)\right) \\
& F=\Delta^{*} \cup\left(\bigcup_{N^{+}} C_{l}^{+}(x, \xi)\right) \cup\left(\bigcup_{N^{-}} C_{l}^{-}(x, \xi)\right), \\
& F=\Delta^{*} \cup\left(\bigcup_{N^{+}} C^{+}(x, \xi)\right) \cup\left(\bigcup_{N^{-}} C^{-}(x, \xi)\right)
\end{aligned}
$$

where $N^{+}$and $N^{-}$are closed cones with $N^{+} \cup N^{-}=N$, no interior point of $N^{+}$(resp. $N^{-}$) belongs to $N^{-}$(resp. $N^{+}$), and in case (c) $N^{+}$and $N^{-}$are invariant under the Hamilton flow. We shall now show that $E$ is not uniquely determined $\bmod C^{\infty}$ by the condition $W F^{\prime}(E) \subset F$ except when $N^{+}$and $N^{-}$are disjoint, which is the case discussed in Theorem 
6.5.3. The case (c) is left for section 6.6 but cases (a) and (b) follow from a supplement to Theorem 6.2.1:

Proposition 6.5.11. Let $P$ satisfy the hypotheses of Theorem 6.5.3. For any $s \in \mathbf{R}$ and $(x, \xi) \in N,(y, \eta) \in T^{*}(X)$ one can choose $K \in \mathscr{D}^{\prime}(X \times X)$ so that $K \in H_{(t)}(X \times X)$ when $t<s$ and either

(a) $P K \in C^{\infty}(X \times X)$ and $W F^{\prime}(K)=W F_{(s)}^{\prime}(K)=$ cone generated by $B(x, \xi) \times\{(y, \eta)\}$, or

(b) $K P \in C^{\infty}(X \times X)$ and $W F^{\prime}(K)=W{F_{(s)}^{\prime}}^{\prime}(K)=$ cone generated by $\{(y, \eta)\} \times B(x, \xi)$.

Proof. It suffices to consider (a). As in the proof of Lemma 6.5.5 the only new point is that the operator $P \otimes 1$ in $\mathscr{D}^{\prime}(X \times X)$ is not a pseudodifferential operator. However, we can choose a pseudo-differential operator $R$ in $X \times X$ such that $W F(R)$ contains no point of the form $\left(x^{\prime}, 0, y^{\prime}, \eta^{\prime}\right)$ and in addition $W F(I-R)$ does not meet $B(x, \xi) \times\{(y, \eta)\}$. Then $(P \otimes 1) R$ is a pseudodifferential operator which has $B(x, \xi) \times\{(y,-\eta)\}$ as a bicharacteristic strip. If we choose $K \in \mathscr{D}^{\prime}(X \times X)$ with $K \in H_{(t)}(X \times X)$ for $t<s$ so that $W F(K)=W F_{(s)}(K)$ is generated by $B(x, \xi) \times\{(y,-\eta)\}$ and $(P \otimes 1) R K \in C^{\infty}$, which is possible by Theorem 6.2.1, then the fact that $(I-R) K \in C^{\infty}$ gives that $(P \otimes 1) K \in C^{\infty}$. But $(P \otimes 1) K$ is the kernel of the composition $P K$.

Using Proposition 6.5.11 we also find that a restriction on the wave front set of a right parametrix does not guarantee that it is a parametrix except in the case discussed in Theorem 6.5.3.

THEOREM 6.5.12. Let $P$ satisfy the hypotheses of Theorem 6.5 .3 and let $E$ be a right parametrix of $P$ with $W F^{\prime}(E) \subset\left(T^{*}(X) \backslash 0\right) \times\left(T^{*}(X) \backslash 0\right)$. Unless $E$ is the parametrix $E_{v}^{+}$of Theorem 6.5.3 for some $\nu \subset \tilde{N}$ there exists another right parametrix $E_{1}$ with $W F^{\prime \prime}\left(E_{1}\right) \subset$ $W F^{\prime}(E)$ which is not a left parametrix.

Proof. If $(x, \xi, y, \eta) \in W F^{\prime}(E) \backslash\left(\Delta^{*} \cup C\right)$ it follows from Lemma 6.5.5 that $B(x, \xi) \times$ $\{(y, \eta)\} \subset W F^{\prime}(E)$. Choose $K$ according to part (a) of Proposition 6.5.11. $E+K$ is then a right parametrix with $W F^{\prime}(E+K) \subset W F^{\prime}(E)$ but $E$ and $E+K$ cannot both be left parametrices since $K P$ is not in $C^{\infty}$ by the observations following Lemma 6.5.5. Assume now that $W F^{\prime}(E) \subset \Delta^{*} \cup C$. If $N^{+}$and $N^{-}$are defined as in the proof of Theorem 6.5.8 it follows from Theorem 6.5.6 that $W F^{\prime}(E)=F$ is given by (6.5.12a). If $N^{+}$and $N^{-}$are disjoint we have the situation of Theorem 6.5.3. On the other hand, if $(x, \xi) \in N^{+} \cap N^{-}$then $B(x, \xi) \times$ $\{(x, \xi)\} \subset W F^{\prime}(E)$ so we can again use Proposition 6.5.11 to find a right parametrix $E_{1}$ with $W F^{\prime}\left(E_{1}\right) \subset W F^{\prime}(E)$ which is not a left parametrix.

Parametrices with entirely different properties can exist for operators which have real principal symbol but do not satisfy the hypotheses of Theorem 6.3.3. In particular, 
this is the case if we allow $P$ to have periodic bicharacteristic strips but require that $C$ is a closed submanifold of $T^{*}(X \times X) \backslash 0$, i.e., in each component we have either a fibration by periodic bicharacteristic strips or else no periodic bicharacteristic strips at all. We can then use Remark 3 at the end of section 6.4 provided that the subprincipal part of $P$ satisfies the non-resonance condition described there. Moreover, in the case that all bicharacteristic strips are periodic the right parametrix $E$ can be chosen properly supported, so it follows as in the proof of Proposition 2.5.1 that it is also a left parametrix and that right and left parametrices are uniquely determined modulo $C^{\infty}$. It follows also that $P u \in$ $C^{\infty}(X)$ implies $u \in C^{\infty}(X)$ for all $u \in \mathscr{D}^{\prime}(X)$. However, as soon as bicharacteristics occur the operator $P$ is not hypoelliptic in the usual local sense that sing supp $u \subset \operatorname{sing} \operatorname{supp} P u$ for all $u \in \mathscr{D}^{\prime}(X)$. In fact, by Theorem 6.2.1 hypoellipticity implies that all bicharacteristic strips stay over a fixed point in $X$, so $p_{\xi}^{\prime}(x, \xi)=0$ whenever $p(x, \xi)=0$. Since we assume that $d p \neq 0$ when $p=0$ it follows that the rank of the differential of the projection $N \rightarrow X$ is constant equal to $n-1$, which implies that locally $p(x, \xi)=\varphi(x) q(x, \xi)$ in neighborhoods of the zeros of $p$. Here $q \neq 0$ and $\varphi_{x}^{\prime} \neq 0$. However, for such operators we have $p_{x}^{\prime}=\varphi_{x}^{\prime} q$ on $N$. The orbits of the Hamilton flow on $N$ are therefore straight lines in the fibers so they cannot be periodic. This proves the statement.

For example, the operator $P$ in Remark 2 after Theorem 6.3.1 has all the properties required above, even if we let $P$ be defined on the whole plane including the origin.

\subsection{The distinguished parametrices}

Theorem 6.5.12 and the discussion preceding Proposition 6.5.11 underline the particular interest of the parametrices constructed in Theorem 6.5.3. These will be called distinguished parametrices here. As mentioned in the preface the case of the Klein-Gordon operator $\square+m^{2}$ in $\mathbf{R}^{n}$ has been considered in relativistic quantum mechanics. The set $\tilde{N}$ has two elements then. When $v=\tilde{N}$ or $\nu=\varnothing$ one can take for $E_{\nu}^{+}$the Feynman "propagator" or its complex conjugate, while we obtain the advanced or retarded fundamental solutions when $v$ has one element. This covers all the $2^{2}$ cases. Note that modulo $C^{\infty}$ we have thus in Theorem 6.5.3 given an analogue of the Feynman propagator for the wave operator on a pseudo-Riemannian structure of Lorentz signature. We do not see how to fix the indetermination. (See also DeWitt [34].)

If $v_{1}$ and $v_{2}$ are disjoint subsets of $N$ then

$$
E_{v_{1}}^{+}+E_{v_{2}}^{+}=E_{v_{1} \nu_{2}}^{+}+E_{\phi}^{+} \bmod C^{\infty} .
$$

In fact, $E=E_{v_{1}}^{+}+E_{v_{2}}^{+}-E_{v_{1} v_{2}}^{+}$is a parametrix, and if we apply Theorem 6.5.7 to the difference of the last two terms it follows that 


$$
W F^{\prime}(E) \cap C^{+} \subset\left(N_{v_{1}}^{+} \times N_{\nu_{1}}^{+}\right) \cap C^{+} .
$$

By the symmetry we can interchange indices 1 and 2 which shows that

hence that $E=E_{\phi}^{+} \bmod C^{\infty}$.

$$
W F^{\prime}(E) \subset \Delta^{*} \cup C^{-},
$$

When $\tilde{n} \in N$ we shall write $C_{\tilde{n}}=C \cap(\tilde{n} \times \tilde{n})$ (where $\tilde{n}$ is considered as a component of $N$ ) and write

$$
S_{\tilde{n}}=E_{\{\tilde{n}\}}^{+}-E_{\phi}^{+} \in I_{1}^{+}-m\left(X \times X, C_{\tilde{n}}^{\prime}\right)
$$

For any subset $\nu$ of $N$ we have then

$$
E_{\nu}^{+}=E_{\phi}^{+}+\sum_{\tilde{n} \in \nu} S_{\tilde{n}} \quad\left(\bmod C^{\infty}\right)
$$

This makes sense even if $\nu$ is not finite for on any compact subset of $X \times X$ only finitely many terms are not in $C^{\infty}$. Hence

so

$$
\begin{aligned}
E_{\nu}^{-}=E_{\phi}^{+}+\sum_{\tilde{n} \notin \nu} S_{\tilde{n}} & =E_{\tilde{N}}^{+}-\sum_{\tilde{n} \in \nu} S_{\tilde{n}}=E_{\phi}^{-}-\sum_{\tilde{n} \in \nu} S_{\tilde{n}} \\
E_{\phi}^{-}-E_{\phi}^{+} & =E_{\tilde{N}}^{+}-E_{\phi}^{+}=\sum_{\tilde{N}} S_{\tilde{n}} .
\end{aligned}
$$

In view of Theorem 6.5.3 and (6.6.3) this shows that $W F^{\prime}\left(S_{\tilde{n}}\right)=C_{\tilde{n}}$. It follows that $E_{\phi}^{+}$and $S_{\tilde{n}}, \tilde{n} \in \tilde{N}$, are linearly independent $\bmod C^{\infty}$ and that only $k+1$ of the $2^{k}$ distinguished parametrices are linearly independent $\bmod C^{\infty}$. Here $k=\operatorname{card} \tilde{N}$.

We shall now compute the principal symbol of $S_{\tilde{n}}$. For the case $P=D_{n}$ this was done in Proposition 6.1.2, part (iii). To study the general case we shall first show that there is a natural density on $C$ and a trivialization of $L_{C}$, both of which are invariant under canonical transformations.

$C$ can be regarded as a fiber space $C \rightarrow N / C$ with base $N / C$ by taking $B(x, \xi) \times B(x, \xi)$ as the fiber through the point $(x, \xi, y, \eta) \in C$. The parameter in the Hamilton equations defined as 0 at $(x, \xi)$ gives an inclusion $B(x, \xi) \subset \mathbf{R}$. If $(x, \xi)$ is replaced by another element in the same class of $N / C$ we just obtain a translation of $B(x, \xi)$ on $\mathbf{R}$. Thus the Lebesgue measure on $\mathbf{R}^{2}$ defines a density in the fibers of $C \rightarrow N / C$. Furthermore $N / C$ is a symplectic manifold, for the tangent space can be identified with the tangent space of $N$ at some point modulo the direction of the Hamilton field there. (See also section 3.3, p. 161). The fiber product $d_{C}(p)$ of the two densities is the required density in $C$. Note that if $p_{1}$ and $p_{2}$ are the liftings of $p$ to $\left(T^{*}(X) \backslash 0\right) \times\left(T^{*}(X) \backslash 0\right)$ by the two projections, then the Lie derivatives $\mathscr{L}_{H_{p_{j}}} d_{C}(p)=0$. In the particular case where $P=D_{n}$ we can identify $N / C$ with the cotangent space of $\mathbf{R}^{n-1}$ (which again proves that $N / C$ is symplectic in view of Proposition 6.1.3). If we use the parametrization (6.1.3) of $C$ then $d_{C}$ is $d x^{\prime} d \theta d x_{n} d y_{n}$. 
The definition of $d_{\sigma}(p)$ is obviously invariant under canonical transformations. We shall now consider the effect of replacing $p$ by $q=a p$ where $a$ is real valued and $\neq 0$. This does not change $C$ but since $H_{q}=a H_{p}$ on the characteristics the parameters on the bicharacteristic strips are affected. If $t$ and $s$ denote the parameters on the bicharacteristic strips of $p$ and $q$ then $\partial / \partial s=a \partial / \partial t$ or equivalently $\partial t / \partial s=a$. On the diagonal $\Delta_{N}$ of $N \times N$ we therefore have

$$
d_{C}(q)=a^{-2} d_{C}(p), \text { that is, } \sqrt{d_{C}(q)}=|a|^{-1} \sqrt{d_{C}(p)} .
$$

$\sqrt{d_{C}(p)}$ is a homogeneous section of $\Omega_{\frac{1}{2}}(C)$ of degree $(n-1) / 2-(m-1)$. In fact, by (6.6.5) it suffices to prove this when $m=1$. Since the Hamilton field is invariant under multiplication by positive scalars the statement follows then from the fact that the symplectic measure in a conic symplectic manifold of dimension $2 k$ is homogeneous of degree $k$.

We shall now verify that the bundle $L_{C}$ is trivial (even as a $\mathbf{Z}$ bundle). Since $\Delta_{N}$ is a deformation retract of $C$ it suffices to prove this for the restriction of $L_{C}$ to $\Delta_{N}$, and we may assume that $P$ is of degree 1 . When $P=D_{n}$ we want to have the trivialization given by the phase function $\left\langle x^{\prime}-y^{\prime}, \theta\right\rangle$ occurring in Proposition 6.1.2. In addition we want to have a trivialization which is invariant under canonical transformations. If $\left(x_{0}, \xi_{0}\right) \in N$ we therefore take according to Proposition 6.1.3 a canonical transformation from a conic neighborhood of $\left(x_{0}, \xi_{0}\right)$ to a conic neighborhood of $\left(X_{0}, \Xi_{0}\right) \in T^{*}\left(\mathbf{R}^{n}\right) \backslash 0$. Let $\psi(x, X, \Theta), \Theta \in \mathbf{R}^{n} \backslash 0$, be a phase function defining this canonical transformation. The proof of Theorem 4.2.2 shows that near $\left(x_{0}, \xi_{0}, x_{0}, \xi_{0}\right)$ the canonical relation $C$ is defined by the phase function

$$
\varphi\left(x, y ; X, Y, \Theta_{X}, \Theta_{Y}, \theta\right)=\psi\left(x, X, \Theta_{X}\right)+\left\langle X^{\prime}-Y^{\prime}, \theta\right\rangle-\psi\left(y, Y, \Theta_{Y}\right)
$$

where $\sigma=\left(X, \Theta_{X}, \theta, Y, \Theta_{Y}\right) \in \mathbf{R}^{5 n-1}$ is to be considered as the fiber variable, and $(x, y) \epsilon$ $X \times X$. We have written $X=\left(X^{\prime}, X_{n}\right)$ and $Y=\left(Y^{\prime}, Y_{n}\right)$. Thus

$$
C \supset\left\{\left(x, \varphi_{x}^{\prime}, y,-\varphi_{y}^{\prime}\right) ; \varphi_{X}^{\prime}=\varphi_{\Theta_{X}}^{\prime}=\varphi_{\theta}^{\prime}=\varphi_{Y}^{\prime}=\varphi_{\Theta_{Y}}^{\prime}=0\right\} .
$$

When $x=y$ is near $x_{0}$ and $\varphi_{x}^{\prime}=-\varphi_{y}^{\prime}$ is near $\xi_{0}$ it follows from the preceding conditions that $X=Y$ and $\Theta_{X}=\Theta_{Y}$. Since interchange of $\left(x, X, \Theta_{X}\right)$ and $\left(y, Y, \Theta_{Y}\right)$ changes the sign of $\varphi$ it follows that the signature of $\varphi_{\sigma \sigma}^{\prime \prime}$ is equal to 0 . Thus one can define $C$ locally near $\Delta_{N}$ by non-degenerate phase functions $\varphi(x, y, \sigma)$ such that $\operatorname{sgn} \varphi_{\sigma o}^{\prime \prime}=0$ at all points corresponding to $\Delta_{N}$. This proves the triviality of $L_{C}$.

At a point in $\Delta_{N}$ where $p_{\xi}^{\prime} \neq 0$ the rank of the projection $C \rightarrow X \times X$ is $n+1$ whereas it is $n-1$ at points with $p_{\xi}^{\prime}=0$. For the phase function constructed above we therefore have $5 n-1-$ rank $\varphi_{\sigma \sigma}^{\prime \prime}=2 n-(n \pm 1)=n \mp 1$ (Theorem 3.1.4). Thus we can always reduce the number of fiber variables as explained in sections 3.1 and 3.2 to $n+1$, that is, by $4 n-2$ 
units. This can be done so that the signature of the new phase function is also 0 whereas the rank is 0 or 2 . Summing up: At $\Delta_{N}$ we can define $C$ by non-degenerate phase functions $\varphi(x, y, \theta)$ with $\theta \in \mathbf{R}^{n+2} \backslash 0$ such that $x=y, \varphi_{\theta}^{\prime}=0, \varphi_{x}^{\prime}=\varphi_{y}^{\prime}$ implies $\operatorname{sgn} \varphi_{\theta \theta}^{\prime \prime}=0$ and rank $\varphi_{\theta \theta}^{\prime \prime}=0$ or 2 . The corresponding trivialization of $L_{C}$ (see the paragraph following Theorem 3.3.4) is invariant under canonical transformations and will be used throughout here.

THЕОВЕМ 6.6.1. Let $P$ be of real principal type in $X$ and assume that $X$ is pseudo. convex. The principal symbol of $S_{\tilde{n}} \in I_{1}^{\mathbf{t}^{-m}}\left(X \times X, C_{\tilde{n}}^{\prime}\right)$ pulled back to $C_{\tilde{n}}$ by the map $C_{\tilde{n}} \rightarrow C_{\tilde{n}}^{\prime}$ is then in $C_{\tilde{n}}$ equal to

$$
i \sqrt{2 \pi d_{C}(p)} a
$$

where $a$ is the function on $C$ which is equal to 1 on $\Delta_{N}$ and satisfies the differential equations

$$
i^{-1} H_{p_{1}} a+c_{1} a=0, \quad i^{-1} H_{p_{2}} a-c_{2} a=0 .
$$

Here $p_{j}$ and $c_{j}$ are the liftings of the principal part $p$ and the subprincipal part $c$ (see (5.2.8)) to $\left(T^{*}(X) \backslash 0\right) \times\left(T^{*}(X) \backslash 0\right)$ by the projection to the first or second copy of $T^{*}(X) \backslash 0$. If $f$ is a solution of the equation $H_{p} f+c=0$ and $f_{j}$ are the two liftings, then $a=\exp i\left(f_{1}-f_{2}\right)$.

Proof. We shall first prove that (6.6.6) is valid with $a=\mathrm{l}$ on $\Delta_{N}$. By (6.6.5) this statement is invariant under multiplication of $P$ by an elliptic factor with positive principal part so we may assume that $P$ is of degree 1. Since in the proof of Theorem 6.5.3 the wave front set of $G^{ \pm}$does not meet $\Delta_{N}$ the assertion follows by inspection of that proof and Proposition 6.1.2. To prove (6.6.6) off the diagonal we note that $P S_{\tilde{n}} \in C^{\infty}$ and compute the principal symbol using Theorem 5.3.1. Since $\mathscr{L}_{H_{p_{1}}} \sqrt{d_{C}(p)}=0$ this gives

$$
i^{-1} H_{p_{1}} a+c a \in S_{1}^{m-2} \text {, or } H_{\mathfrak{p}_{2}}\left(a e^{-i f f_{1}}\right) \in S_{1}^{m-2} \text {, }
$$

which completes the proof.

When $c$ is real the integral operator in $B(x, \xi) \times B(x, \xi),(x, \xi) \in N$, with kernel equal to the function $a$ in Theorem 6.6.1 is positive and of rank 1 . This suggests that $S_{\tilde{n}} / i$ might be a positive operator when $c$ is real, that is, when $P^{*}-P \in L_{1}^{m-2}(X)$. Here $P^{*}$ is the adjoint of $P$,

$$
(P u, v)=\left(u, P^{*} v\right) ; u, v \in C_{0}^{\infty}(X) .
$$

(Recall that $u, v$ are densities of order $\frac{1}{2}$ so the sesquilinear scalar products used here are intrinsically defined.) However, the fact that the principal symbol only gives rise to an operator of rank 1 indicates that the situation is highly unstable so that lower order terms in $P$ may play a role. This is confirmed by the following example. Let $X=\mathbf{R}^{n}$ and let $P$ 
have the symbol $\xi_{n}+i c\left|\xi^{\prime}\right|^{-v}$ in a conic neighborhood of $\xi_{n}=0 ; c$ is a real constant and $v$ a positive integer. We can write the parametrix $E^{+}$modulo a psendo-differential operator with $\xi_{n} \neq 0$ in its wave front set as

$$
E^{+} u(x)=\iint e^{i\left\langle x^{\prime}-y^{\prime}, \theta\right\rangle} a^{+}\left(x_{n}, y_{n}, \theta\right) u(y) d y d \theta
$$

Here $a^{+}\left(x_{n}, y_{n}, \theta\right)=0$ for $x_{n}<y_{n}, a^{+}\left(y_{n}+0, y_{n}, \theta\right)=i$ and

$$
D_{x_{n}} a^{+}\left(x_{n}, y_{n}, \theta\right)+i c|\theta|^{-v} a^{+}\left(x_{n}, y_{n}, \theta\right)=0 \text {. }
$$

This gives

$$
a^{+}\left(x_{n}, y_{n}, \theta\right)=i \exp \left(c\left(x_{n}-y_{n}\right)|\theta|^{-v}\right) x_{n}>y_{n} \text {. }
$$

Similarly we can construct $E^{-}$and obtain

$$
i^{-1}\left(E^{+}-E^{-}\right) u(x)=\iint e^{i\left\langle x^{\prime}-y^{\prime}, \theta\right\rangle} a\left(x_{n}, y_{n}, \theta\right) u(y) d y d \theta
$$

where $a\left(x_{n}, y_{n}, \theta\right)=\exp \left(c\left(x_{n}-y_{n}\right)|\theta|^{-v}\right)$ for all $x_{n}, y_{n}$. Now choose $u(y)=v\left(y^{\prime}\right) w\left(y_{n}\right)$ where $w$ is fixed in $C_{0}^{\infty}$ and $\int w\left(x_{n}\right) d x_{n}=0, \int x_{n} w\left(x_{n}\right) d x_{n}=1$. Then

$$
\iint a\left(x_{n}, y_{n}, \theta\right) w\left(x_{n}\right) \overline{w\left(y_{n}\right)} d x_{n} d y_{n}=-|\theta|^{-2 v} c^{2}+\ldots
$$

where dots indicate a symbol of order $-3 \nu$. Hence

$$
\operatorname{Re}\left(\left(E^{+}-E^{-}\right) i^{-1} u, u\right) \leqslant-c^{2} / 2\|v\|_{(-v)}^{2}+(R u, u)
$$

where $R$ has a $C^{\infty}$ kernel (see section 2.2), so no operator in the same class as $\left(E^{+}-E^{-}\right) / i$ $\bmod C^{\infty}$ has a positive real part. This motivates the hypothesis that $P$ is self-adjoint in the following theorem.

THEОR EM 6.6.2. Let $P$ be self-adjoint and of real principal type in $X$, and assume that $X$ is pseudo-convex. Then one can choose $S_{\tilde{n}}$ anti-self-adjoint so that

$$
\left(i^{-1} S_{\tilde{n}} u, u\right) \geqslant 0, u \in C_{0}^{\infty}(X)
$$

Proof. Taking the adjoint of the equation $P E^{+}=I+R$, where $R$ has a $C^{\infty}$ kernel, we obtain $\left(E^{+}\right)^{*} P=I+R^{*}$. Since $W F\left(E^{+}\right) \subset \Delta^{*} \cup C^{+}$it follows that $W F^{\prime}\left(\left(E^{+}\right)^{*}\right) \subset \Delta^{*} \cup C^{-}$ so $\left(E^{+}\right)^{*}-E^{-} \in C^{\infty}$. Taking adjoints in (6.6.4) we therefore obtain

$$
\sum\left(S_{\tilde{n}}+S_{\tilde{n}}^{*}\right) \in C^{\infty}
$$

and since the terms have disjoint wave front sets each of them is in $C^{\infty}$. Hence $S_{\tilde{n}}$ can be normalized so that $S_{\tilde{n}} / i$ is self-adjoint. 
The proof of the positivity is much harder except in the case $P=D_{n}$ when we obtain from $(6.1 .1)$

$$
\left(i^{-1}\left(E_{n}^{+}-E_{n}^{-}\right) u, u\right)=(2 \pi)^{-(n-1)} \int|\hat{v}(\theta)|^{2} d \theta=\int\left|v\left(x^{\prime}\right)\right|^{2} d x^{\prime}
$$

where

$$
v\left(x^{\prime}\right)=\int u\left(x^{\prime}, x_{n}\right) d x_{n}
$$

In order to reduce the general case to this one we have to refine Propositions 6.1.3 and 6.1.4 so that we can construct $S_{\tilde{n}}$ in one stroke in a conic neighborhood in $\left(T^{*}(X) \backslash 0\right) \times$ $\left(T^{*}(X) \backslash 0\right)$ of the inverse image in $C$ of a neighborhood of any point in $N / C$. In doing so we shall also obtain the tools for completing the proof of Theorem 6.5 .8 , for example, so at first we shall not require $P$ to be self-adjoint.

Let $A$ be an invertible elliptic operator of order $(1-m) / 2$ with homogeneous principal symbol and set $Q=A^{*} P A$. Then the first order operator $Q$ is of order 1 and has the same bicharacteristic relation as $P$; if $P$ is self-adjoint so is $Q$. If $E^{ \pm}$are distinguished parametrices of $Q$ it follows that $A E^{ \pm} A^{*}$ are distinguished parametrices of $P$. Since $A$ and $A^{*}$ preserve wave front sets we obtain from the parametrix differences $S_{\tilde{n}}$ corresponding to $Q$ the analogous ones $A S_{\tilde{n}} A^{*}$ corresponding to $P$. Thus it suffices to consider the operator $Q$ instead of $P$. In view of condition (iv) in Theorem 6.4.3 it follows that we can take the principal symbol of $A$ so small at infinity in $X$ that in the representation of $N$ given by that condition $M^{\prime}=M_{0} \times \mathbf{R}$. In other words, the parameter on every bicharacteristic strip of $Q$ runs from $-\infty$ to $\infty$. This is a notational convenience only.

Summing up, we shall assume in the following lemmas that $P$ is of order 1 , and that the Hamilton field $H_{p}$ on $N=\left\{(x, \xi) \in T^{*}(X) \backslash 0, p(x, \xi)=0\right\}$ satisfies the conditions in Theorem 6.4.3 with $M^{\prime}=M_{0} \times \mathbf{R}$ in (iv).

LEMMA 6.6.3. For every $\left(x_{0}, \xi_{0}\right) \in N$ there is an open conic neighborhood $U$ in $T^{*}(X) \backslash 0$ and a homogeneous canonical transformation

$$
U \ni(x, \xi) \rightarrow \chi(x, \xi)=\left(X_{1}(x, \xi), \ldots, X_{n}(x, \xi), \Xi_{1}(x, \xi), \ldots, \Xi_{n}(x, \xi)\right) \in T^{*}\left(\mathbf{R}^{n}\right) \backslash 0
$$

mapping $U$ bijectively on an open conic neighborhood $U^{\prime}$ of $\chi\left(x_{0}, \xi_{0}\right)=\left(0, \Xi_{0}\right)$, such that.

(i) $p(x, \xi)=\Xi_{n}(x, \xi)$,

(ii) $U^{\prime} \cap N^{\prime}$, where $N^{\prime}=\left\{(X, \Xi) \in T^{*}\left(\mathbf{R}^{n}\right) \backslash 0, \Xi_{n}=0\right\}$, is invariant under translations along the $X_{n}$ axis, and $U^{\prime}$ is symmetric with respect to the plane $X_{n}=0$ and convex in the direction of the $X_{n}$ axis.

Since $H_{p}$ is transformed to $\partial / \partial X_{n}$ by $\chi^{\prime}$ it follows from (ii) that $U \cap N$ is also invariant under $H_{p}$. 
Proof. By Proposition 6.1.3 we can find a local canonical transformation with all the listed properties except that $U \cap N$ and $U^{\prime} \cap N^{\prime}$ are not invariant under the Hamilton fields. By integration along the Hamilton fields $H_{p}$ and $\partial / \partial X_{n}$ we can extend $U, U^{\prime}$ and $\chi$ in the required way. In fact, by hypothesis the integration gives a bijection between the flowouts of $U \cap N$ and of $U^{\prime} \cap N^{\prime}$ and since exponentiation of the Hamilton field gives a canonical transformation we obtain a canonical bijection of a neighborhood. This proves the lemma.

Let $U_{1}$ and $U_{2}$ be closed conic neighborhoods of $\left(x_{0}, \xi_{0}\right)$ such that $U_{1} \subset U$ and $U_{2}$ is in the interior of $U_{1}$ while $U_{j} \cap N$ are invariant under the Hamilton flow. We may take $U_{j}$ so that $U_{j}^{\prime}=\chi U_{j}$ are convex in the $X_{n}$-direction and symmetric about the plane $X_{n}=0$. By $\Gamma_{1}$ and $\Gamma_{2}$ we denote the graph of the restriction of $\chi$ to $U_{1}$ and to $U_{2}$.

LEм м 6.6.4. There exists a Fourier integral operator $A \in I_{1}^{0}\left(X \times \mathbf{R}^{n}, \Gamma_{1}^{\prime}\right)$ such that

(i) $A$ is non-characteristic in $\Gamma_{2}^{\prime}$,

(ii) $\Gamma_{2} \cap W F^{\prime}\left(P A-A D_{n}\right)=\varnothing$.

If $P$ is self-adjoint we can choose $A$ so that in addition

(iii) $W F\left(A^{*} A-I\right) \cap U_{2}^{\prime}=\varnothing$

or which is equivalent

(iii)' $W F\left(A A^{*}-I\right) \cap U_{2}=\varnothing$.

Proof. The first part of the statement follows by inspection of the proof of Proposition 6.1.4 since for every $a \in S_{1}^{k}\left(U_{2}^{\prime}\right)$ one can find $b \in S_{1}^{k}\left(U_{2}^{\prime}\right)$ with $\partial b / \partial X_{n}=a$ and $b=0$ when $X_{n}=0$. When $P$ is self-adjoint it follows from (ii) by taking adjoints that

(ii) ${ }^{\prime} \Gamma_{2}^{-1} \cap W F^{\prime}\left(A^{*} P-D_{n} A^{*}\right)=\varnothing$

Hence it follows that

$$
U_{2}^{\prime} \cap W F\left(A^{*} P A-D_{n} A^{*} A\right)=\varnothing, U_{2}^{\prime} \cap W F^{\prime}\left(A^{*} P A-A^{*} A D_{n}\right)=\varnothing
$$

and so

$$
W F\left(\left[A^{*} A, D_{n}\right]\right) \cap U_{2}^{\prime}=\varnothing .
$$

Thus the full symbol of $A^{*} A$ is the sum of one term of order $-\infty$ near $U_{2}^{\prime}$ and one which does not depend on $X_{n}$ and is bounded from below near $U_{2}^{\prime}$. From the proof of Proposition 2.2.2 it follows that one can find $B \in L_{1}^{0}\left(\mathbf{R}^{n}\right)$ properly supported with $W F(B) \subset U_{1}^{\prime}$ such that

$$
W F\left(B^{*} B-A^{*} A\right) \cap U_{2}^{\prime}=\varnothing \text { and } W F\left(\left[B, D_{n}\right]\right) \cap U_{2}^{\prime}=\varnothing .
$$

The proof of Proposition 2.5.1 shows that one can find $A_{1} \in L_{1}^{0}\left(\mathbf{R}^{n}\right)$ properly supported with $W F\left(A_{1}\right) \subset U_{1}^{\prime}$ and 
$W F\left(A_{1} B-I\right) \cap U_{2}^{\prime}=\varnothing$, hence $W F\left(B A_{1}-I\right) \cap U_{2}^{\prime}=\varnothing$

Since

$W F\left(A_{1}^{*} B^{*} B A_{1}-A_{1}^{*} A^{*} A A_{1}\right) \cap U_{2}^{\prime}=\varnothing, \quad W F\left(A_{1}\left[B, D_{n}\right] A_{1}\right) \cap U_{2}^{\prime}=\varnothing$

this implies that

$$
W F\left(I-\left(A A_{1}\right)^{*} A A_{1}\right) \cap U_{2}^{\prime}=\varnothing, \quad W F\left(\left[A_{1}, D_{n}\right]\right) \cap U_{2}^{\prime}=\varnothing
$$

From (ii) we now obtain

$$
\varnothing=\Gamma_{2} \cap W F^{\prime}\left(P A A_{1}-A D_{n} A_{1}\right)=\Gamma_{2} \cap W F^{\prime}\left(P A A_{1}-A A_{1} D_{n}\right)
$$

since $\Gamma_{2} \cap W F^{\prime}\left(A\left[D_{n}, A_{1}\right]\right)=\varnothing$. The proof is complete.

As shown in section 5.1 we can always choose $B \in I_{1}^{0}\left(\mathbf{R}^{n} \times X,\left(\Gamma_{1}^{-1}\right)^{\prime}\right)$ so that

$$
W F(A B-I) \cap U_{2}=\varnothing, \quad W F(B A-I) \cap U_{2}^{\prime}=\varnothing
$$

In the self-adjoint case we shall of course take $B=A^{*}$, but we do not make this hypothesis yet. Modulo $C^{\infty}$ a one to one correspondence between pseudo-differential operators $T \in L_{1}^{0}(X)$ and $T^{\prime} \in L_{1}^{0}\left(\mathbf{R}^{n}\right)$ with $W F(T) \subset U_{2}$ and $W F\left(T^{\prime}\right) \subset U_{2}^{\prime}$ is obtained by writing

$$
T=A T^{\prime} B, \quad T^{\prime}=B T A
$$

(We take all pseudo-differential operators properly supported.) In fact,

$$
A(B T A) B-T=(A B-I) T A B+T(A B-I) \in C^{\infty}
$$

and similarly $B\left(A T^{\prime} B\right) A-T^{\prime} \in C^{\infty}$. For general $T$ and $T^{\prime}$ in $L_{1}^{0}$ it is still true that

$$
W F(A(B T A) B-T) \cap U_{2}=\varnothing, \quad W F\left(B\left(A T^{\prime} B\right) A-T^{\prime}\right) \cap U_{2}^{\prime}=\varnothing .
$$

The correspondence (6.6.11) preserves algebraic operations: If $T_{j}^{\prime}=B T_{j} A, j=1,2,3$, then

In fact,

$$
W F\left(T_{1} T_{2}-T_{3}\right) \cap U_{2}=\varnothing \Leftrightarrow W F\left(T_{1}^{\prime} T_{2}^{\prime}-T_{3}^{\prime}\right) \cap U_{2}^{\prime}=\varnothing
$$

$$
T_{1}^{\prime} T_{2}^{\prime}-T_{3}^{\prime}=B T_{1} A B T_{2} A-B T_{3} A=B\left(T_{1} T_{2}-T_{3}\right) A+B T_{1}(A B-I) T_{2} A
$$

and the wave front set of the last term does not meet $U_{2}^{\prime}$. Similarly,

In fact,

$$
W F([T, P]) \cap N \cap U_{2}=\varnothing \Leftrightarrow W F\left(\left[T^{\prime}, D_{n}\right]\right) \cap N^{\prime} \cap U_{2}^{\prime}=\varnothing .
$$

$$
\left[T^{\prime}, D_{n}\right]=\left[B T A, D_{n}\right]=B T\left(A D_{n}-P A\right)+B[T, P] A+\left(B P-D_{n} B\right) T A
$$

By (ii) in Lemma 6.6.4 the wave front set of the first term does not meet $U_{2}^{\prime}$ and the same 
is true for the last term since again by (ii) $W F\left(P-A D_{n} B\right) \cap U_{2}=\varnothing$, hence $W F^{\prime}(B D-$ $\left.D_{n} B\right) \cap \Gamma_{2}^{-1}=\varnothing$.

If $S$ is the difference $E_{n}^{+}-E_{n}^{-}$which occurs in (6.6.9) and if $U_{2} \cap N$ is in the component $\tilde{n}$ of $N$, then

$$
W F^{\prime}\left(S_{\tilde{n}}-A S B\right) \cap\left(U_{2} \times U_{2}\right)=\varnothing .
$$

Indeed, the proof of Theorem 6.5.3 shows that $W F^{\prime}\left(A E_{n}^{ \pm} B-E^{ \pm}\right)$does not meet the diagonal in $\left(U_{2} \cap N\right) \times\left(U_{2} \cap N\right)$. Hence the same is true of $W F^{\prime}\left(A S B-S_{\tilde{n}}\right)$ which is also contained in C. However,

$$
P\left(A S B-S_{\tilde{n}}\right)=\left(P A-A D_{n}\right) S B,
$$

so $W F^{\prime}\left(P\left(A S B-S_{\tilde{n}}\right)\right)$ does not meet $U_{2} \times U_{2}$ by (ii) in Lemma 6.6.4. Hence it follows from Lemma 6.5.5 and the remark following it that (6.6.14) is valid. This leads to an important step in the proof of Theorem 6.6.2:

Lемма 6.6.5. If $P$ is self-adjoint, $T \in L_{1}^{0}(X)$ and $W F([T, P]) \cap N=\varnothing$, then

$$
S_{\tilde{n}} T T^{*}-T A S A^{*} T^{*} \in C^{\infty}
$$

if $W F(T) \subset U_{2}$ and $N \cap U_{2}$ is in the component $\tilde{n}$. (The notations are those of Lemma 6.6.4.)

Proof. Taking $B=A^{*}$ we have by (6.6.14) and the invariance of $U_{2} \cap N$

$$
S_{\tilde{n}} T T^{*}-A S A^{*} T T^{*} \in C^{\infty} \text {. }
$$

Now introduce $T^{\prime}=A^{*} T A$ following (6.6.11). Then $W F\left(T^{\prime}\right) \subset U_{2}^{\prime}$ and by (6.6.13)

$$
W F\left(\left[T^{\prime}, D_{n}\right]\right) \cap N^{\prime}=\varnothing \text {. }
$$

Thus the derivative with respect to $x_{n}$ of the symbol of $T^{\prime}$ is of order $-\infty$ in a neighborhood of $N^{\prime}$. If we denote by $\tau_{h}$ convolution by the Dirac measure at $(0, \ldots, 0, h)$, then $S=\int \tau_{h} d h$, and $\tau_{h} T^{\prime} \tau_{-h}-T^{\prime}$ is of order $-\infty$ in a conic neighborhood of $N^{\prime}$. If $u \in \mathscr{E}^{\prime}\left(\mathbf{R}^{n}\right)$ it follows that

$$
W F\left(\tau_{h} T^{\prime} u-T^{\prime} \tau_{h} u\right) \cap N^{\prime}=\varnothing
$$

for $N^{\prime}$ is invariant under translations in the $x_{n}$ direction. Since $T^{\prime}$ is properly supported an integration with respect to $h$ gives

$$
W F^{\prime}\left(\left[S, T^{\prime}\right] u\right) \cap N^{\prime}=\varnothing .
$$

On the other hand, $W F\left(\left[S, T^{\prime}\right] u\right) \subset N^{\prime}$ since $W F^{\prime}(S) \subset N^{\prime} \times N^{\prime}$ so we conclude that $\left[S, T^{\prime}\right] u \in C^{\infty}$, hence that $\left[S, T^{\prime}\right] \in C^{\infty}$. Thus

$$
A^{*} T A S-S A^{*} T A \in C^{\infty},
$$

and if we multiply by $A$ to the left and by $A^{*} T^{*}$ to the right we obtain

$$
T A S A^{*} T^{*}-A S A^{*} T T^{*} \in C^{\infty} \text {. }
$$

In view of (6.6.16) this proves $(6.6 .15)$. 
By (6.6.9) the operator $T A S A^{*} T^{*} / i$ is positive. To prove Theorem 6.6.2 it therefore remains only to show that the identity can be written as a sum of operators of the form $T T^{*}$ discussed in Lemma 6.6.5. In doing so we again drop for a moment the hypothesis that $P$ is self-adjoint. We keep the notations of Lemma 6.6.4 and take in addition a closed conic neighborhood $U_{3}$ of $\left(x_{0}, \xi_{0}\right)$ contained in the interior of $U_{2}$ such that $U_{3} \cap N$ is also invariant under the Hamilton flow.

LEмма 6.6.6. There exists an operator $T \in L_{1}^{0}(X)$ with $W F(T) \subset U_{2}$ such that $T$ has a non-negative homogeneous principal symbol equal to 1 in $U_{3}$ and

$$
W F([T, P]) \cap N=\varnothing .
$$

Proof. Introducing $T=A T^{\prime} B$ as in (6.6.11) we reduce the proof to finding $T^{\prime} \in L_{1}^{0}\left(\mathbf{R}^{n}\right)$ with $W F\left(T^{\prime}\right) \subset U_{2}^{\prime}$, such that the principal symbol is homogeneous and equal to 1 in $U_{3}^{\prime}=\chi U_{3}$, and

$$
W F\left(\left[T^{\prime}, D_{n}\right)\right] \cap N^{\prime}=\varnothing \text {. }
$$

In the proof we need another closed conic neighborhood $V$ of $\left(0, \Xi_{0}\right)$ such that $V \cap N^{\prime}$ is invariant under the vector field $\partial / \partial x_{n}$ and $U_{3}^{\prime}$ (resp. $V$ ) is in the interor of $V$ (resp. $U_{2}^{\prime}$ ). Choose a non-negative $C^{\infty}$ function $\varphi_{0}\left(x^{\prime}, \xi\right)$ which is homogeneous of degree 0 , vanishes outside $\left\{\left(x^{\prime}, \xi\right) ;\left(x^{\prime}, 0, \xi\right) \in V\right\}$ and is 1 in a neighborhood of $\left\{\left(x^{\prime}, \xi\right) ;\left(x^{\prime}, 0, \xi\right) \in U_{3}\right\}$. Then choose $\varphi_{1}(x, \xi)$ homogeneous of degree 0 , equal to 1 in a neighborhood of $V$ and 0 outside $U_{2}^{\prime}$. The product $\varphi(x, \xi)=\varphi_{0}\left(x^{\prime}, \xi\right) \varphi_{1}(x, \xi)$ is independent of $x_{n}$ in a neighborhood of $N^{\prime}$ so a pseudo-differential operator $T^{\prime}$ with symbol $\varphi$ has the required properties.

End of proof of Theorem 6.6.2. Choose a countable number of triples of closed cones $U_{1}^{j} \supset U_{2}^{j} \supset U_{3}^{j}$ and corresponding operators $A_{j}$ as above so that $U_{2}^{j} \cap N \subset \tilde{n}$ for every $j$ and $\bigcup U_{3}^{j} \supset \tilde{n}$. In addition we require that the projections $\pi U_{2}^{j}$ in $X$ have closed neighborhoods $F_{j}$ such that the sets $F_{j}$ are locally finite. Choose corresponding operators $T_{j} \in L_{1}^{0}(X)$ with $W F\left(T_{j}\right) \subset U_{2}^{\prime}$ according to Lemma 6.6.6, thus $W F\left(\left[P, T_{j}\right]\right) \cap N=\varnothing$. We can take $T_{j}$ so that the kernel vanishes outside $F_{j} \times F_{j}$. Then the sum

$$
G=\sum T_{j} T_{j}^{*}
$$

is defined, the principal symbol of $G$ is positive in a neighborhood of $\tilde{n}$ and $W F(G) \cap N=\tilde{n}$. Moreover, $W F([G, P]) \cap N=\varnothing$. Proposition 2.2.2 or rather its proof shows that we can find a self-adjoint $R \in L_{1}^{0}(X)$ with non-negative principal symbol and $W F\left(G-R R^{*}\right) \cap N=\varnothing$. We claim that

$$
W F([R, P]) \cap N=\varnothing
$$

This is obvious if $P=D_{n}$ for $\mathbb{W} F\left(\left[G, D_{n}\right]\right) \cap N=\varnothing$ means that some symbol of $T$ is inde- 
pendent of $x_{n}$ in a neighborhood of the characteristic set and it is obvious that the sums used to define $R$ will have the same properties. (Note that the conditions on $R$ determine the symbol $\bmod S^{-\infty}$ in a conic neighborhood of $N$.) By the remarks following Lemma 6.6.4 we obtain the same conclusion for a general $P$.

Now choose $R_{1} \in L_{1}^{0}$ so that $W F\left(R R_{1}-I\right) \cap \tilde{n}=\varnothing$. Then it follows that

If $\tilde{T}_{j}=R_{1} T_{j}$ it follows that

$$
W F\left(\left[R_{1}, P\right]\right) \cap \tilde{n}=\varnothing .
$$

$$
W F\left(\tilde{T}_{j}\right) \cap N \subset \tilde{n}, \quad W F\left(I-\sum \tilde{T}_{j} \tilde{T}_{j}^{*}\right) \cap \tilde{n}=\varnothing, \quad W F\left(\left[P, \tilde{T}_{j}\right]\right) \cap N=\varnothing .
$$

Using Lemma 6.6.5 we obtain with congruences modulo $C^{\infty}$

$$
S_{\tilde{n}} \equiv \sum S_{\tilde{n}} \tilde{T}_{j} \tilde{T}_{j}^{*} \equiv \sum \tilde{T}_{j} A_{j} S A_{j}^{*} \tilde{T}_{j}^{*}
$$

Hence we can define $S_{\tilde{n}}$ to be the right hand side, and since every term there is positive after division by $i$, the theorem is proved.

We shall now give a supplement to Lemma 6.5.9 which will show that (6.5.10), (6.5.11) can be fulfilled and therefore completes the proof of Theorem 6.5.8. (The reduction to operators $P$ satisfying the hypotheses made here is left for the reader.)

LEMм A 6.6.7. Let $N_{1}, \ldots, N_{k}$ be closed conic subsets of $N$ with union $N$ which are all invariant under the Hamilton flow, and let $\frac{1}{2}<\varrho<1$. Then one can find $T_{1}, \ldots, T_{k} \in L_{\varrho}^{0}(X)$ with $T_{1}+\ldots+T_{k}=I$ and

$$
W F\left(T_{j}\right) \cap N \subset N_{f}, W F\left(\left[P, T_{j}\right]\right) \cap N=\varnothing, \quad j=1, \ldots, k .
$$

Proof. If $P=D_{n}$ the assertion follows at once from Lemma 6.5 .9 applied to $\Gamma_{j}=$ $\left\{(x, \xi) ;\left(x, \xi^{\prime}, 0\right) \in N_{j} \cup 0\right\}$. In fact, we can take the operators in Lemma 6.5.9 of the form $a_{i}\left(x^{\prime}, D\right)$ then so the commutator with $D_{n}$ is 0 . In the general case we can transplant such operators to the manifold using (6.6.11) and obtain $T_{1}, \ldots, T_{k}$ with $W F\left(T_{j}\right) \cap N \subset N_{j}$, $W F\left(\left[P, T_{j}\right]\right) \cap N=\varnothing$ and

$$
W F\left(I-T_{1}-\ldots-T_{k}\right) \cap U_{2}=\varnothing
$$

Now choose a covering of $N$ as in the proof of Theorem 6.6.2 but with $\tilde{n}$ replaced by $N$, and choose corresponding operators $\tilde{T}_{j} \in L_{1}^{0}$ with $W F\left(\widetilde{T}_{j}\right) \subset U_{2}^{j}$ and $W F\left(I-\Sigma \tilde{T}_{j}\right) \cap N=\varnothing$, $W F\left(\left[P, \tilde{T}_{j}\right]\right) \cap N=\varnothing$. For each $j$ we can choose $T_{1}^{j}, \ldots, T_{k}^{j}$ as above. If we set

$$
T_{i}=\sum_{j} \tilde{T}_{j} T_{i}^{j}, i=1, \ldots, k
$$

it follows that $T_{1}, \ldots, T_{k}$ have all required properties except that only $W F\left(I-\sum_{1}^{k} T_{i}\right) \cap N=$ $\varnothing$ instead of $I=\Sigma T_{i}$. However, if we replace $T_{1}$ by $I-\Sigma_{2}^{k} T_{i}$ the proof is complete. 16-722909 Acta mathematica 128. Imprimé le 28 Mars 1972. 
Finally we shall supplement Proposition 6.5.11 with a result which shows that there are many parametrices with wave front set contained in the set given by (6.5.12 c) unless it is the wave front set of a distinguished parametrix.

Proposition 6.6.8. Let $P$ satisfy the hypotheses of Theorem 6.5.3. For arbitrary $\left(x_{j}, \xi_{j}\right) \in N, j=1,2$, one can choose $K \in \mathscr{D}^{\prime}(X \times X)$ so that $W F^{\prime}(K)$ is the cone generated by $B\left(x_{1}, \xi_{1}\right) \times B\left(x_{2}, \xi_{2}\right)$ and $P K \in C^{\infty}, K P \in C^{\infty}$.

Proof. For $j=1,2$ we choose conic neighborhoods $U_{1}^{j}, U_{2}^{f}$ of $\left(x_{j}, \xi_{j}\right)$ and operators $A_{1}^{j}, B_{1}^{j}$ as in Lemma 6.6.4 and the following discussion. Let $\left(0, \Xi_{0}^{j}\right) \in T^{*}\left(\mathbf{R}^{n}\right) \backslash 0$ be the point corresponding to $\left(x_{j}, \xi_{j}\right)$, thus $\Xi_{0}^{j}=\left(\theta_{j}, 0\right)$ where $\theta_{j} \in \mathbf{R}^{n-1} \backslash 0$. Now choose $K_{1} \in \mathscr{D}^{\prime}\left(\mathbf{R}^{n-1} \times \mathbf{R}^{n-1}\right)$ with $W F^{\prime \prime}\left(K_{1}\right)=\left\{\left(0, t \theta_{1} ; 0, t \theta_{2}\right), t>0\right\}$. If $K$ is regarded as a distribution in $\mathbf{R}^{n} \times \mathbf{R}^{n}$ independent of the $n$th coordinates, Theorem 2.5.11' shows that

$$
W F^{\prime}\left(K_{1}\right)=\left\{\left(0, X_{n}, t \Xi_{0}^{1} ; 0, Y_{n}, t \Xi_{0}^{2}\right) ; t>0, X_{n}, Y_{n} \in \mathbf{R}\right\} .
$$

Since $D_{n} K_{1}=K_{1} D_{n}=0$ the composition $K=A_{1} K_{1} B_{2}$ has the required properties.

We shall end this section with some brief additional comments.

Remark 1. All solutions of the equation $D_{n} u=0$ can be written in the form $u=\left(E_{n}^{+}-\right.$ $\left.E_{n}^{-}\right) v$ for some $v$ such that the projection of supp $v$ on the plane $x_{n}=0$ along the $x_{n}$ axis is proper. The quadratic form $(S v, v) / i$ only depends on $u$ and not on $v$ and may be thought of as the energy of $u$. Using the partitions of unity above it is easy to show that if $P u=$ $f \in C^{\infty}$ and $W F(u) \subset \tilde{n}$ then we have $u=S_{\tilde{n}} v \bmod C^{\infty}$ for some $v$. We leave for the reader to supply the proofs and the precise conditions on the supports of $v$ and $S_{\tilde{n}}$.

Remark 2. It is usually necessary to work $\bmod C^{\infty}$ to obtain the results proved above, even if there is a natural choice of $E_{v}^{+}$. For example, if $m^{2}$ is replaced by $-m^{2}$ in the KleinGordon equation the $E_{v}^{+}$are unambiguously defined but they are all linearly independent, the differences corresponding to $S_{\tilde{n}}$ do not only depend on $\tilde{n}$, they are not skew adjoint and do not have positive imaginary part. This follows easily by writing down Fourier integral representations of $E_{v}^{+}$. Again we leave the details for the reader.

\section{Pseudo-differential operators with complex principal symbols}

\subsection{Introduction}

In this chapter we shall study pseudo-differential operators $P \in L_{1}^{m}(X)$ where $X$ is a $C^{\infty}$ manifold, assuming that $P$ has a homogeneous principal symbol $p$. According to [17, Chap. VI] it is necessary to require that 


$$
\{p, \bar{p}\}=0 \quad \text { when } p=0
$$

in order to have even local existence of solutions of the equation $P u=f$ when $P$ is a differential operator. In the case of pseudo-differential operators the same condition is necessary in order to have an existence theorem both for $P$ and for ${ }^{t} P$. (See [42].) We shall therefore always assume that (7.0.1) is fulfilled. In addition we shall assume that the Hamilton fields $H_{\mathrm{Re} p}, H_{\mathrm{Im} p}$ are linearly independent when $p=0$. (For many local results it is of course sufficient to make these assumptions locally.) From (7.0.1) it follows then that $\{\operatorname{Re} p, \operatorname{Im} p\}=a \operatorname{Re} p+b \operatorname{Im} p$ for some smooth $a$ and $b$. In view of the Jacobi identity this implies that

$$
\left[H_{\mathrm{Re} p}, H_{\mathrm{Im} p}\right]=a H_{\mathrm{Re} p}+b H_{\mathrm{Im} p} \text { when } p=0
$$

By the Frobenius theorem we therefore have through every characteristic point a twodimensional manifold contained in the characteristics and tangent to the vector fields $H_{\text {Re } y}$ and $H_{\operatorname{Im} p}$. In analogy with the real case the manifold will be called a bicharacteristic strip. This is natural since we prove theorems on existence and regularity of solutions which then become very close to the corresponding results in the real case. The main difference is from the analytic point of view that for solutions of $P u=f \in C^{\infty}$ the order of differentiability is no longer constant along the bicharacteristics but instead a superharmonic function with respect to the analytic structure defined by the Hamilton field $H_{p}$. Geometrically the new features are caused by the fact that we have to consider two dimensional foliations and these may have a much more complicated (semi-)global behavior than one dimensional foliations.

In section 7.1 we begin with a local and (semi-)global discussion of first order differential operators satisfying the preceding conditions. Using a local transformation of a general operator to the Cauchy-Riemann operator with parameters we extend in section 7.2 the local results to theorems on the propagation of singularities of solutions of the equation $P u=f$. From these we also derive sufficient conditions for local or (semi-)global existence theorems. In section 7.3 we then resume the discussion of first order differential operators. In particular we study the case of cone manifolds which is required for differential equations involving symbols. This prepares for the construction in section 7.4 of solutions of $P u=f \in C^{\infty}$ with given superharmonic degree of differentiability in the wave front set, which is the cone generated by a bicharacteristic strip, and for the construction of parametrices in section 7.5. In contrast to the real case the existence theorems obtained via a parametrix are weaker than those obtained from the local arguments in section 7.2. Also in other respects the results in this chapter are not as complete as those in chapter VI. For example only right (or left) parametrices are constructed. 


\subsection{First order differential operators}

Let $M$ be a $C^{\infty}$ manifold and let $L$ be a complex $C^{\infty}$ vector field on $M$, i.e., $L=L_{1}+i L_{2}$ where $L_{1}$ and $L_{2}$ are real $C^{\infty}$ vector fields on $M$. We assume that

$$
\begin{gathered}
{\left[L_{1}, L_{2}\right](x) \text { is a linear combination of } L_{1}(x) \text { and } L_{2}(x) \text { for all } x \in M,} \\
L_{1}(x) \text { and } L_{2}(x) \text { are linearly independent for every } x \in M .
\end{gathered}
$$

These conditions are invariant under multiplication of $L$ by a non-vanishing complex valued function. If more generally $L$ is a first order differential operator from complex valued functions on $M$ to sections of a complex line bundle on $M$ and $L I=0$ conditions (7.1.1) and (7.1.2) can therefore still be applied to $L$. All that follows remains valid in this slightly more general context.

Our purpose is to discuss the existence of smooth solutions $u$ of

$$
L u=f
$$

when $f$ is smooth. Condition (7.1.1) is necessary for the local existence even of a distribution solution as remarked in the introduction. Together (7.1.1) and (7.1.2) are sufficient for the local existence of a smooth solution. To see this we first note that by the Frobenius theorem one can choose local coordinates near 0 for example so that $L_{1}$ and $L_{2}$ are linear combinations of $\partial / \partial x_{n-1}$ and $\partial / \partial x_{n}$. Thus

$$
L=a_{n-1} \partial / \partial x_{n-1}+a_{n} \partial / \partial x_{n}
$$

can be regarded as an elliptic operator in the variables $x^{\prime \prime}=\left(x_{n-1}, x_{n}\right)$ depending on the parameters $x^{\prime}=\left(x_{1}, \ldots, x_{n-2}\right)$. It follows that there is a parametrix

$$
E f(x)=(2 \pi)^{-2} \iint e^{i\left\langle x^{\prime \prime}-y^{\prime \prime}, \theta\right\rangle} a(x, \theta) f\left(x^{\prime}, y^{\prime \prime}\right) d y^{\prime \prime} d \theta
$$

in a neighborhood $U$ of 0 . Thus $L E f=f-R f, f \in \mathscr{E}^{\prime}(U)$, where $R$ has a $C^{\infty}$ kernel. If $\chi \in C_{0}^{\infty}\left(\mathbf{R}^{n}\right)$ is equal to 1 in a neighborhood of 0 and $\chi_{\varepsilon}(x)=\chi(x / \varepsilon)$ we obtain a solution of (7.1.3) near 0 by taking $u=E g$ where $g-\chi_{\varepsilon} R g=\chi_{\varepsilon} f$. For small $\varepsilon$ it is clear that the Neumann series

$$
g=\sum_{\mathbf{0}}^{\infty}\left(\chi_{\varepsilon} R\right)^{j} \chi_{\varepsilon} f
$$

converges to a function in $C_{0}^{\infty}(U)$ which proves the local existence of solutions of (7.1.3). (This is of course a special case of the theorems concerning operators of constant strength in $[17$, Chap. VII].) 
From (7.1.1) and (7.1.2) it follows that for some $\lambda \in C^{\infty}(M)$

$$
[L, \bar{L}]=\lambda L-\bar{\lambda} \bar{L} .
$$

If $\mu$ is a (local) solution of $L \bar{\mu}=\bar{\lambda}$ and $L^{\prime}=e^{\mu} L$, then

$$
\left[L^{\prime}, \bar{L}^{\prime}\right]=e^{2 \operatorname{Re} \mu}([L, \bar{L}]+(L \bar{\mu}) \bar{L}-(\bar{L} \mu) L)=0 .
$$

An application of the Frobenius theorem now gives local coordinates such that $L^{\prime}=$ $\partial / \partial x_{n-1}+i \partial / \partial x_{n}$ so we have proved

Lемма 7.1.1. At every point in $M$ there is a local coordinate system such that $L=a \partial / \partial \bar{z}$ where $a \in C^{\infty}$ is different from 0 and $\partial / \partial \bar{z}=\left(\partial / \partial x_{n-1}+i \partial / \partial x_{n}\right) / 2$ is the Cauchy-Riemann operator in $x_{n-1}+i x_{n}$.

If $y_{1}, \ldots, y_{n}$ is another local coordinate system for which $L$ takes this simple form, then $y^{\prime}$ is a function of $x^{\prime}$ and $y_{n-1}+i y_{n}$ is an analytic function of $x_{n-1}+i x_{n}$ when the other variables are fixed. Thus there is a natural analytic structure in the leaves of the $L_{1}, L_{2}$ foliation-or $L$ foliation for short. (For basic definitions and facts concerning foliations see Haefliger [40].) This analytic structure allows us to consider differentials of type $(j, k)$ along the foliation for arbitrary $j$ and $k$; in terms of the local coordinates in Lemma 7.1.1 these are of the form $a d z^{j} d \bar{z}^{k}$ with $a \in C^{\infty}$. Here $d z$ and $d \bar{z}$ shall anticommute. When $j=0$ (or $k=0$ ) we make the invariant definition $\partial\left(a d \bar{z}^{k}\right)=\partial a / \partial z d z d \bar{z}^{k} \quad$ (resp. $\bar{\partial}\left(a d z^{j}\right)=$ $\left.\partial a / \partial \bar{z} d \bar{z} d z^{j}\right)$. Now we can write

$$
L=a \bar{\partial}
$$

where $a$ is a differential of type $(0,-1)$ with no zeros. (The existence of such a differential is a topological restriction which could be avoided by allowing the range of $L$ to consist of sections of a line bundle.) From (7.1.6) and $\bar{L}=\bar{a} \partial$ we obtain

$$
L \bar{L}=a \bar{a} \partial \bar{\partial}+\bar{b} \bar{L}, \quad \bar{L} L=a \bar{a} \partial \bar{\partial}+b L
$$

where $b$ is a function which in view of (7.1.5) must be equal to $-\lambda$. Hence

$$
a \bar{a} \partial \bar{\partial}=(L+\bar{\lambda}) \bar{L}=(\bar{L}+\lambda) L .
$$

If $\varphi$ is defined in an open subset of a leaf of the foliation we recall that $\varphi$ is called subharmonic if $\varphi$ is upper semicontinuous and $\partial \bar{\partial} \varphi \geqslant 0$ in the sense of distribution theory (unless $\varphi=-\infty$ identically). By (7.1.7) this is equivalent to either of the conditions $(L+\bar{\lambda}) \bar{L} \varphi \geqslant 0$ or $(\bar{L}+\lambda) L \varphi \geqslant 0$. When $\varphi \in C^{2}$ we say that $\varphi$ is strictly subharmonic if strict pointwise inequalities are valid. When $\varphi$ is defined in $M$ we say that $\varphi$ is subharmonic if $\varphi$ is upper 
semicontinuous in $M$ and the restriction to each leaf is subharmonic. Finally $\varphi$ is called superharmonic if $-\varphi$ is subharmonic.

The local description of $L$ in Lemma 7.1.1 leads easily to a rather precise description of the singularities and the supports of solutions of (7.1.3). To measure the smoothness of a distribution $u$ in $M$ we set

$$
s_{u}(x)=\sup \left\{t \in \mathbf{R} ; u \in H_{(t)} \text { in a neighborhood of } x\right\} .
$$

(In section 7.2 we shall also consider an analogous function in the cotangent bundle.) Note that $s_{u}$ is lower semicontinuous by definition.

Leмм 7.1.2. If $u \in \mathscr{D}^{\prime}(M)$ and $L u=f$, then $\min \left(s_{u}, s\right)$ is a superharmonic function if $s$ is superharmonic and $s_{f} \geqslant s$.

Proof. The statement is local so in view of Lemma 7.1.1 we may assume that $M$ is an open subset of $\mathbf{R}^{n}$ and that $L=\partial / \partial \bar{z}, z=x_{n-1}+i x_{n}$. We just have to prove that if $h$ is a harmonic function in $\mathbf{C}$ such that

$$
\min \left(s_{u}, s\right)(0, z)>h(z), \quad|z|=r,
$$

then the inequality is valid when $|z| \leqslant r$ if $(0, z) \in M$ then. It is obvious that $s(0, z)>h(z)$ when $|z| \leqslant r$. If $v=\chi_{1} \chi_{2} u$ and $\chi_{1} \in C_{0}^{\infty}\left(\mathbf{R}^{n-2}\right)$ has support in a small neighborhood of 0 , $\chi_{1}(0)=1$, while $\chi_{2} \in C_{0}^{\infty}(\mathbf{C})$ is 1 for $|z| \leqslant r$ and 0 outside a small neighborhood, then $\partial v / \partial \bar{z}=g$ and $s_{g}>h$ since

$$
g=\chi_{1} \chi_{2} f+\chi_{1} \partial \chi_{2} / \partial \bar{z} u
$$

Let $F$ be an analytic function in $\mathbf{C}$ with $\operatorname{Re} F=h$ and denote by $Q$ the pseudodifferential operator $\left(1+|D|^{2}\right)^{F(z) / 2}$. Then we have $\partial(Q v) / \partial \bar{z}=Q g$, and $Q g \in L_{10 \mathrm{loc}}^{2}$ since for every $x$ and $\varrho<1$ we have $Q \in L_{\varrho}^{t}$ in a neighborhood of $x$ if $h\left(x^{\prime \prime}\right)<t$. If $\chi \in C_{0}^{\infty}\left(\mathbf{R}^{n}\right)$ is equal to 1 in a neighborhood of $\operatorname{supp} v$ we conclude that $\partial w / \partial \bar{z} \in L^{2}$ if $w=\chi Q v$. An application of Cauchy's integral formula (or Fourier transformation) now shows that $w \in L^{2}$, hence that $Q v \in L_{\text {loc }}^{2}$. It follows that $v \in H_{(t)}$ in a neighborhood of $x$ if $t<h(x)$. Hence $s_{u} \geqslant h$ at $(0, z)$ when $|z| \leqslant r$ which proves the lemma.

Remark. If $a \in C^{\infty}(M)$ and $(L+a) u=f$ the same conclusion is valid. In fact, the assertion is local and if $b$ is a local solution of $L b=a$ we can apply the lemma to the equation $L\left(u e^{b}\right)=f e^{b}$ instead.

When $f \in C^{\infty}$ we can take $s=+\infty$ and conclude that $s_{u}$ is superharmonic. It will be shown in section 7.3 that this conclusion cannot be improved, and a general version of the lemma will be given in section 7.2.

In the following propositions $a$ denotes a function in $C^{\infty}(M)$. 
Propositron 7.1.3. Let $u \in \mathscr{D}^{\prime}(M)$ and $(L+a) u=f$. Then sing supp $u \backslash \operatorname{sing} \operatorname{supp} f$ is invariant under the L-foliation in $\mathbf{C} \operatorname{sing} \operatorname{supp} f$, i.e., if $x \in \operatorname{sing} \operatorname{supp} u \backslash \operatorname{sing} \operatorname{supp} f$ then the whole leaf through $x$ of the L-foliation in $\mathbf{C}$ sing supp $f$ is contained in sing supp $u$.

Proof. The statement follows by applying Lemma 7.1.2 in $M_{1}=C$ sing supp $f$ and noting that a superharmonic function which is equal to $+\infty$ in an open subset $\Omega$ must be equal to $+\infty$ in each leaf through $\Omega$.

Proposition 7.1.4. Let $u \in \mathscr{D}^{\prime}(M)$ and $(L+a) u=f$. Then $\operatorname{supp} u \backslash \operatorname{supp} f$ is invariant under the L-foliation in $\mathbf{C}$ supp $f$.

Proof. This follows from the classical theorem on unique continuation of analytic functions since we obtain smoothness from Proposition 7.1.3 and can remove the term $a$ as in the remark above.

Remark. The preceding propositions follow from Theorems 8.8.1 and 8.9.1 in [17] but we have preferred to give direct elementary proofs making this paper selfcontained.

We shall now show that the preceding results lead to semi-global and global existence theorems for the equation (7.1.3).

THEOREM 7.1.5. Let $K$ be a compact subset of $M$ and denote by $C^{\infty}(K)$ the quotient of $C^{\infty}(M)$ by the subspace of elements vanishing of infinite order on $K$. Then the following conditions are equivalent:

(a) $L C^{\infty}(K)=C^{\infty}(K)$.

(b) $(L+a) C^{\infty}(K)=C^{\infty}(K)$ for every $a \in C^{\infty}(K)$.

(c) $L C^{\infty}(K)$ and $(L+\bar{\lambda}) C^{\infty}(K)$ are dense in $C^{\infty}(K)$, where $\lambda$ is the function in (7.1.5).

(d) There exists a function $\varphi \in C^{\infty}(K)$ which is strictly subharmonic in $K$.

(e) No leaf of the L-foliation is contained in $K$.

Proof. It is trivial that $(a) \Rightarrow(b) \Rightarrow(c)$. If (c) is valid we first choose $f \in C^{\infty}(K)$ with $(\bar{L}+\lambda) f$ so close to 1 that $\operatorname{Re}(\bar{L}+\lambda) f>\frac{1}{2}$ on $K$. Then choose $\varphi \in C^{\infty}(K)$ with $L \varphi$ so close to $f$ that $\operatorname{Re}(\bar{L}+\lambda) L \varphi>0$ on $K$. By (7.1.7) it follows that (d) is valid for $\operatorname{Re} \varphi$. (Note that (c) means that the $\bar{\partial}$ operator has a dense range when acting on smooth functions as well as on smooth $(1,0)$ forms on $K$ so the condition is quite natural.)

Now suppose that (d) is valid and let $B$ be a leaf of the $L$-foliation. If $B \subset K$ then $\bar{B} \subset K$ and $\bar{B}$ contains the leaf through any one of its points. At a point in $\bar{B}$ where $\varphi$ is maximal the strictly subharmonic function $\varphi$ has a maximum on the corresponding leaf which is a contradiction. 
Finally assume that (e) is valid. Then (a) follows by repetition of the proof of Theorem 6.3.1 if we show that

$$
\begin{gathered}
v \in \mathscr{E}^{\prime}(K),{ }^{t} L v=0 \Rightarrow v=0, \\
v \in \mathscr{E}^{\prime}(K),{ }^{t} L v=f, s_{f} \geqslant t \in \mathbf{R} \Rightarrow s_{u} \geqslant t .
\end{gathered}
$$

Here ${ }^{t} L$ is the adjoint of $L$ with respect to some $C^{\infty}$ density in $M$ so ${ }^{t} L=-(L+a)$ for some $a \in C^{\infty}$. Condition (e) gives (7.1.8) when combined with Proposition 7.1.4. By Lemma 7.1.2 and the remark following its proof the hypotheses in (7.1.9) imply that $S=\min \left(s_{u}, t\right)$ is superharmonic and that $S \leqslant t$ with equality in $C K$. Thus the upper semi-continuous function $S$ assumes its minimum at some point $x \in K$ and $S$ is therefore constant in the leaf $B$ containing $x$. By (e) some point in $C K$ is in $B$ so $\min S=S(x)=t$ which proves (7.1.9) and the theorem.

Theorem 7.1.5 shows that semi-global solvability, i.e. $L C^{\infty}(K)=C^{\infty}(K)$ for every compact set $K \subset M$, is equivalent to

No leaf of the $L$-foliation is relatively compact in $M$.

Now suppose that we have semi-global solvability. Then a slight modification of Théorème 2, p. 328 in Malgrange [48] shows that $L C^{\infty}(M)=C^{\infty}(M)$ if and only if

For each compact subset $K$ of $M$ and integer $k \geqslant 0$ there is a compact set $K^{\prime} \subset M$ such that $v \in \mathscr{E}^{\prime}(M),{ }^{t} L v \in \mathscr{E}^{\prime} k(K)$ implies $v \in \mathscr{E}^{\prime}\left(K^{\prime}\right)$.

In view of Proposition 7.1.4 the condition (a) in the next theorem together with (7.1.10) is therefore sufficient for global solvability.

THEOREM 7.1.6. Suppose that $L C^{\infty}(K)=C^{\infty}(K)$ for every compact set $K \subset M$. Then the following conditions are equivalent:

(a) For each compact set $K \subset M$ there exists a compact set $K^{\prime} \subset M$ such that if $B$ is a leaf of the $L$-foliation and $C$ a component of $B \cap C K$ in the leat topology which is relatively compact in the M-topology, then $C \subset K^{\prime}$.

(b) For each compact set $K \subset M$ there exists a compact set $K^{\prime} \subset M$ such that for every $x \notin K^{\prime}$ there is a solution $f \in C^{\infty}(M)$ of $L f=0$ with the property

$$
|f(x)|>\sup _{y \in K}|f(y)| .
$$

(c) There exists a subharmonic function $\varphi \in C^{\infty}(M)$ such that $\{x ; \varphi(x) \leqslant c\}$ is compact in $M$ for every $c \in \mathbf{R}$. 
Proof. Suppose that (a) is valid and choose $K, K^{\prime}$ as in (a). Suppose that $x \notin K^{\prime}$ and that

$$
|f(x)| \leqslant \sup _{y \in \mathbb{K}}|f(y)|
$$

for all $f \in C^{\infty}(M)$ with $L f=0$. By the Hahn-Banach theorem it follows that there is a measure $\mu$ with support in $K$ such that $\mu(f)=f(x)$ for all $f \in C^{\infty}(M)$ with $L f=0$. Thus $\delta_{x}-\mu$ is orthogonal to $\operatorname{Ker} L$ in $C^{\infty}(M)$, hence $\delta_{x}-\mu$ is in the closure of $\operatorname{Im}{ }^{t} L$ in $\mathscr{E}^{\prime}(M)$. (As before we take the adjoint with respect to some smooth positive density which is also used to identify measures with distributions.)

As already pointed out (a) implies that $L C^{\infty}(M)=C^{\infty}(M)$, and because $C^{\infty}(M)$ is a Fréchet space this implies that $\operatorname{Im}^{t} L$ is closed in $\mathscr{E}^{\prime}(M)$ (see Dieudonné-Schwartz [35, Th. 7]). Hence there exists a distribution $\nu \in \mathscr{E}^{\prime}(M)$ such that $\delta_{z}-\mu={ }^{t} L v$. Let $B$ be the leaf through $x$ and $C$ the component of $x$ in $B \cap C K$ in the leaf topology. Because $x \notin K^{\prime}, C$ is not relatively compact in $M$. The same is true for $C \backslash\{x\}$ which is a component of $B \cap \mathcal{C}(K \cup\{x\})$ in the topology of $B$. Since (supp $\left.{ }^{t} L v\right) \cap(C \backslash\{x\})=\varnothing$ and supp $v$ is compact, Proposition 7.1.4 shows that supp $v \cap(C \backslash\{x\})=\varnothing$. But this means that for some neighborhood $\omega$ of $x$ we have ${ }^{t} L v=\delta_{x}$ in $\omega$ and ( $\left.\operatorname{supp} v\right) \cap \omega \subset\{x\}$ which is obviously impossible. This proves (b).

(b) $\Rightarrow$ (c). Since $|f|^{2}$ is subharmonic if $L f=0$ the proof of Theorem 5.1.6 in Hörmander $[41]$ can be applied with no change.

(c) $\Rightarrow$ (a). We have the following maximum principle for subharmonic functions: If $K$ is compact in $M$ and $\varphi$ is subharmonic in a neighborhood of $K$ then

$$
\sup _{x \in \mathbb{R}} \varphi(x)=\sup _{x \in \partial \partial^{\prime} K} \varphi(x)
$$

where $\partial^{\prime} K$ is the boundary of $K$ in the leaf topology. For let the supremum in the left hand side be attained at $y \in K$ and denote the leaf through $y$ by $B$. If $y$ is on the boundary of the closed set $B \cap K$ (in the leaf topology) the assertion is obvious. Otherwise $\varphi$ is constant in the component of the interior of $B \cap K$ (in the leaf topology) containing $y$. This case is ruled out if using Theorem 7.1.5 we choose a strictly subharmonic function $\psi$ near $K$ and replace $\varphi$ by $\varphi+\varepsilon \psi$. Thus (7.1.13) is valid for $\varphi+\varepsilon \psi$ and letting $\varepsilon \rightarrow 0$ we obtain (7.1.13).

Now suppose that $\varphi$ satisfies (c) and that $K$ is compact in $M$. Define $K^{\prime}=\{x \in M$; $\varphi(x) \leqslant c\}$ with $c=\sup \{\varphi(y) ; y \in K\}$. Then $K^{\prime}$ is compact in view of (c). If $C$ is as in (a) then the closure $\bar{C}$ in the $M$-topology is compact and $\partial^{\prime} \bar{C} \subset K$. Therefore $\varphi(x) \leqslant c$ in $C$, hence $C \subset K^{\prime}$. The proof is complete.

Semi-global solvability and condition (c) of Theorem 7.1.6 together are equivalent to

There exists a strictly subharmonic function $\varphi \in C^{\infty}(M)$ such that $\{x \in M ; \varphi(x) \leqslant c\}$ is compact in $M$ for every $c \in \mathbf{R}$. 
Indeed, since semi-global solvability and (c) imply global solvability the proof of (d) in Theorem 7.1.5 gives the existence of a strictly subharmonic function $\psi \in C^{\infty}(M)$. If $\varphi$ is the subharmonic function of condition (c) in Theorem 7.1.6 it follows that $\varphi_{1}=\varphi+e^{\varphi}$ has the properties stated in (7.1.14). Conversely, (7.1.14) implies condition (d) in Theorem 7.1.5 and condition (c) in Theorem 7.1.6.

In analogy with the terminology in complex analysis the manifold $M$ will be called pseudo-convex with respect to $L$ if (7.1.14) is valid. As we have seen pseudo-convexity is equivalent to the conjunction of (7.1.10) and condition (a) in Theorem 7.1.6, and it implies global solvability of $L u=f$ with $C^{\infty}$ solutions. (That (7.1.14) implies global solvability is also easily proved by means of Carleman estimates.) We do not know if the converse is true but we can give a somewhat weaker result:

Proposition 7.1.7. Let $L C^{\infty}(M)=C^{\infty}(M)$ and let $K$ be a compact subset of $M$. Then there exists a compact subset $K^{\prime}$ of $M$ such that, if $B$ is a leaf of the $L$-foliation and $C$ a component of $B \cap \mathrm{C} K$ in the leaf topology which is relatively compact in the leaf topology, it follows that $C \subset K^{\prime}$.

Proof. Let $\varrho$ be a positive $C^{\infty}$ density on $B$ in a neighborhood of $\bar{C}$. Then ${ }^{t} L(v \varrho)=$ $(L v+a v) \varrho$ in a neighborhood of $\vec{C}$ for some $a \in C^{\infty}(M)$. Choose $b \in C^{\infty}(M)$ so that $L b+a=0$ and let $u$ be the product of $e^{b} \varrho$ and the characteristic function of $C$. Then $u \in \mathscr{E}^{\prime 0}(M)$, $\bar{C} \subset \operatorname{supp} u$ and $\operatorname{supp}^{t} L u \subset K$ so the proposition follows from (7.1.11) with $k=1$.

If every open connected subset of a leaf $B$ which is relatively compact in the $M$ topology is also relatively compact in the $B$-topology it follows from Theorem 7.1.6 and Proposition 7.1.7 that pseudo-convexity is necessary and sufficient in order that $L C^{\infty}(M)=$ $C^{\infty}(M)$. In our applications of Theorem 7.1.6 to parametrix constructions we shall have to make much stronger geometric hypotheses than the preceding one for other reasons so the question left open here will not be important.

\subsection{Propagation of singularities and existence theorems}

Let $X$ be an $n$-dimensional manifold and $P \in L_{1}^{m}(X)$ a properly supported pseudodifferential operator with homogeneous principal part $p$. Let $N_{\mathrm{c}}$ be the set of all $(x, \xi) \in T^{*}(X) \backslash 0$ such that $p(x, \xi)=0$ and $H_{\text {Re } p}, H_{\operatorname{Im} p}$ and the direction of the cone axis are linearly independent. This is a $C^{\infty}$ manifold of codimension 2 . The interior relative to $N_{c}$ of the set in $N_{\mathrm{c}}$ where the Poisson bracket $\{p, \bar{p}\}$ vanishes we shall denote by $N$. As pointed out in the introduction the vector fields $H_{\operatorname{Re} p}, H_{\operatorname{Im} p}$ are tangent to $N$ and the tangent system on $N$ spanned by them is integrable. The corresponding foliation will be called the 
bicharacteristic foliation and the leaves bicharacteristic strips. The projection of a bicharacteristic strip in $X$ will be called a bicharacteristic. Note that the projection is non-singular only in the open subset of $N$ where $d_{\xi} \operatorname{Re} p$ and $d_{\xi} \operatorname{Im} p$ are linearly independent.

If $u \in \mathscr{D}^{\prime}(X)$ and $(x, \xi) \in T^{*}(X) \backslash 0$ we shall write

$$
s_{u}^{*}(x, \xi)=\sup \left\{t ; u \in H_{(t)} \text { at }(x, \xi)\right\}
$$

That $u \in H_{(t)}$ at $(x, \xi)$ means that $u=u_{1}+u_{2}$ with $u_{1} \in H_{(t)}$ and $(x, \xi) \notin W F\left(u_{2}\right)$. The function $s_{u}^{*}(x, \xi)$ is lower semi-continuous and homogeneous of degree 0 , and the proof of Theorem 2.5.3 shows that

$$
s_{u}(x)=\inf _{\xi} s_{u}^{*}(x, \xi)
$$

where $s_{u}$ is the function occurring in Lemma 7.1.2.

THEOREM 7.2.1. Let $u \in \mathscr{D}^{\prime}(X), P u=f$, and let $s$ be a positively homogeneous function in an open conic subset $\Omega$ of $N$ with $s \leqslant s_{f}$. Then it follows that $\min \left(s_{u}^{*}, s+m-1\right)$ is superharmonic in $\Omega$ if $s$ is superharmonic in $\Omega$, and that $\min \left(s_{u}^{*}-s, m-1\right)$ is superharmonic in $\Omega$ if $s$ is subharmonic in $\Omega$ (with respect to $H_{p}$ ). In particular, $s_{u}^{*}$ is superharmonic in $\Omega$ if $\Omega \cap W F(f)=\varnothing$.

CoRollaRY 7.2.2. If $u \in \mathscr{D}^{\prime}(X)$ and $P u=f$, then $(N \cap W F(u)) \backslash W F(f)$ is invariant under the bicharacteristic foliation in $N \backslash W F(f)$.

The corollary follows as Proposition 7.1.3 by taking $s=+\infty$ and $\Omega=N \backslash W F(f)$ in Theorem 7.2.1. The proof of Theorem 7.2.1 is parallel to that of Theorem 6.1.1 so we shall first consider the operator $\partial / \partial \bar{z}$ in $\mathbf{R}^{n}, z=x_{n-1}+i x_{n}$, and then pass to the general case by means of Fourier integral operators.

First we shall prove that Lemma 7.1.2 remains valid if $s_{u}, s_{f}$ are replaced by $s_{u}^{*}, s_{f}^{*}$ and $s$ by a function of $x$ and $\xi$ which is homogeneous of degree 0 . In doing so we may assume that $u \in \mathscr{E}^{\prime}\left(\mathbf{R}^{n}\right)$. If $a(\xi)$ is a homogeneous function of degree 0 , then $\partial u / \partial \bar{z}=f$ implies $\partial(a(D) u) / \partial \bar{z}=a(D) f$. We shall apply Lemma 7.1.2 to such equations noting that for every $\xi_{0} \in \mathbf{R}^{n} \backslash 0$ one can choose $a_{j}$ with $a_{j}\left(\xi_{0}\right)=1$ vanishing outside closed cones $\Gamma_{j}$ which decrease to the ray through $\xi_{0}$ as $j \rightarrow \infty$. If $u_{j}=a_{j}(D) u$ we have $s_{u_{j}}(x) \rightarrow s_{u}^{*}\left(x, \xi_{0}\right)$ so the superharmonicity of

$$
\min \left(s_{u_{j}}, s_{j}\right), \text { where } s_{j}(x)=\inf _{\xi \in \Gamma_{j}} s(x, \xi)
$$

gives in the limit that $\min \left(s_{u}^{*}, s\right)$ is superharmonic. This proves the first statement in Theorem 7.2.1 for the operator $\partial / \partial \bar{z}$. To prove the second we first note that it is equivalent to the first if $s$ is harmonic. Hence the second part follows if $s$ is the supremum of 
a family of harmonic functions. If $s \in C^{2}$ is strictly subharmonic, then $s\left(x^{\prime}, z, \xi\right) \geqslant h\left(x^{\prime}, z, \xi\right)$ in a neighborhood of $\left(x^{\prime}, w, \xi\right)$ with equality at $\left(x^{\prime}, w, \xi\right)$ when $h$ is the harmonic function

$$
h\left(x^{\prime}, z, \xi\right)=s\left(x^{\prime}, w, \xi\right)+\operatorname{Re}\left(2(z-w) \partial s\left(x^{\prime}, w, \xi\right) / \partial w+(z-w)^{2} \partial^{2} s\left(x^{\prime}, w, \xi\right) / \partial w^{2}\right)
$$

In view of the local character of superharmonicity this proves the second statement in Theorem 7.2.1 when $s$ is strictly subharmonic and the general case follows by approximation of $s$ with such functions.

In order to pass from $\partial / \partial \bar{z}$ to $P$ we shall use Fourier integral operators corresponding to a canonical transformation obtained from Proposition 6.1.3 with $p_{1}=\operatorname{Re} p$ and $p_{2}=$ $\operatorname{Im} p$. However, this requires that $\{p, \bar{p}\}=2 i\{\operatorname{Re} p, \operatorname{Im} p\}$ vanishes not only on $N$ but in a neighborhood in $T^{*}(X) \backslash 0$. The following lemma gives a reduction to that case.

Lemma 7.2.3. If $\left(x_{0}, \xi_{0}\right) \in N$ one can find a homogeneous $C^{\infty}$ function a of degree $1-m$ with $a\left(x_{0}, \xi_{0}\right) \neq 0$ such that $\{q, \bar{q}\}=0$ in a neighborhood of $\left(x_{0}, \xi_{0}\right)$ if $q=a p$.

Proof. We may assume without restriction that $m=1 . H_{p}$ can then be regarded as a complex vector field on the cosphere bundle so there exists a fixed conic neighborhood $V$ of $\left(x_{0}, \xi_{0}\right)$ such that the equation

$$
\left(H_{p}+a\right) w=f
$$

has a $C^{\infty}$ homogeneous solution of degree 0 in $N \cap V$ for all such $a$ and $f$. The same is true if $w$ and $f$ are taken homogeneous of any degree $\mu$, for we can introduce $w=b w_{1}, f=b f_{1}$ with $b$ homogeneous of degree $\mu$ and different from 0 and obtain an equation of the same form for $w_{1}$ and $f_{1}$. The proof now proceeds in three steps.

1) Assume that $\{p, \bar{p}\}$ vanishes of order $k \geqslant \mathrm{I}$ in $V$ when $p=0$. By Taylor's formula we can then write

$$
\{p, \bar{p}\}=\sum_{0}^{k} a_{j} p^{j} \bar{p}^{k-3},
$$

where we can choose $a_{j}$ so that $\bar{a}_{j}=-a_{k-j}$ since $\{p, \bar{p}\}$ is purely imaginary. We claim that it is possible to choose $w$ vanishing of order $k-1$ when $p=0$ so that if $q=p \exp \bar{w}$ the Poisson bracket $\{q, \bar{q}\}$ vanishes of order $k+1$ in $V$ when $p=0$. To prove this we note that

$$
\{q, \bar{q}\} e^{-w-\bar{w}}=\{p, \bar{p}\}+\bar{p}\{p, w\}+p\{\bar{w}, \bar{p}\}+p \bar{p}\{\bar{w}, w\} .
$$

The last term vanishes of order $2+\max (2 k-3,0) \geqslant k+1$ so it can be ignored. Write

$$
w=\sum_{0}^{k-1} w_{j} p^{j} \bar{p}^{k-1-j}
$$


Then

$$
\{p, w\}=\sum_{0}^{k-1}\left\{p, w_{j}\right\} p^{j} \bar{p}^{k-1-j}+O\left(p^{r}\right)
$$

where $r=\max (k-2+k, 1) \geqslant k$. Thus we must make sure that

$$
\sum_{0}^{k-1}\left(H_{p} w_{j}\right) p^{j} \bar{p}^{k-j}-\sum_{0}^{k-1} \overline{H_{p} w_{j}} p^{k-j} \bar{p}^{\jmath}+\sum_{0}^{k} a_{j} p^{j} \bar{p}^{k-j}=O\left(p^{k+1}\right)
$$

that is,

$$
H_{p} w_{j}-\overline{H_{p} w_{k-j}}+a_{j}=0 \quad \text { in } N \cap V \text { for } j=0, \ldots, k
$$

Here $w_{k}$ should be read as 0 . Since $\bar{a}_{j}=-a_{k-j}$ these equations follow if in $N \cap V$

$$
H_{p} w_{j}+a_{j}=0 \text { when } j<k / 2, \quad w_{j}=0 \text { when } j>k / 2, \quad H_{p} w_{j}+a_{j} / 2=0 \text { if } j=k / 2 .
$$

This proves the assertion.

2) We have now proved that there exist functions $w_{0}, w_{1}, \ldots$ homogeneous of degree 0 and defined in a fixed neighborhood $V$ of $\left(x_{0}, \xi_{0}\right)$ such that if $q_{k}=p \exp \left(\bar{w}_{0}+\ldots+\bar{w}_{k}\right)$ then $\left\{q_{k}, \bar{q}_{k}\right\}$ vanishes of order $k+2$ in $N \cap V$. Moreover, $w_{k}$ vanishes of order $k$ in $N \cap V$. By a classical theorem of $\mathbf{E}$. Borel we can choose a homogeneous $C^{\infty}$ function $w$ such that $w-w_{0}-\ldots-w_{k}$ vanishes of order $k+1$ in $N \cap V$ for every $k$. If $q=p \exp \bar{w}$ it is clear that $\{q, \bar{q}\}$ vanishes of infinite order when $q=0$.

3) We have now reduced the proof to the case where $\{\operatorname{Re} p, \operatorname{Im} p\}$ vanishes of infinite order when $p=0$. Write $p=p_{1}+i p_{2}$ with real $p_{1}, p_{2}$ and set $\left\{p_{1}, p_{2}\right\}=\lambda_{1} p_{1}+\lambda_{2} p_{2}$ where $\lambda_{j} \in C^{\infty}$ vanishes of infinite order in $N \cap V$. Now choose a function $f_{1}$ such that in a conic neighborhood of $\left(x_{0}, \xi_{0}\right)$

$$
\left\{e^{f_{1}} p_{1}, p_{2}\right\}=e^{f_{1}} \lambda_{2} p_{2}, \quad \text { i.e., }\left\{f_{1}, p_{2}\right\}+\lambda_{1}=0
$$

There is a unique solution with initial data $f_{1}=0$ on a conic hypersurface transversal to $H_{p_{2}}$ at $\left(x_{0}, \xi_{0}\right)$ and it is homogeneous of degree 0 . Since $N$ is invariant under the vector field $H_{p_{2}}$ it is clear that $f_{1}$ vanishes of infinite order when $p=0$. Next note that

$$
\left\{e^{f_{1}} p_{1}, e^{f_{2}} p_{2}\right\}=0 \quad \text { if }\left\{e^{f_{1}} p_{1}, f_{2}\right\}+e^{f_{1}} \lambda_{2}=0
$$

This equation also has a solution $f_{2}$ vanishing of infinite order when $p=0$. Thus

$$
a=\left(e^{f_{1}} p_{1}+i e^{f_{2}} p_{2}\right) /\left(p_{1}+i p_{2}\right)=1+\left(\left(e^{f_{1}}-1\right) p_{1}+i\left(e^{f_{2}}-1\right) p_{2}\right) /\left(p_{1}+i p_{2}\right)
$$

is infinitely differentiable and $a-1$ vanishes of infinite order when $p=0$. Since $q=a p=$ $e^{f_{1}} p_{1}+i e^{f_{2}} p_{2}$ the lemma is proved. 
End of proof of Theorem 7.2.1. Let $\left(x_{0}, \xi_{0}\right) \in \Omega$. Using Lemma 7.2.3 we can choose an elliptic operator $E$ of order $1-m$ such that for the principal symbol $q$ of $Q=E P$ we have $\{\operatorname{Re} q, \operatorname{Im} q\}=0$ in a neighborhood of $\left(x_{0}, \xi_{0}\right)$. Since $Q u=E f$ and $s_{E f}^{*}=s_{f}^{*}+m-1$ by the regularity theory of elliptic operators it suffices to prove the theorem for the operator $Q$ instead of $P$. By Proposition 6.1.3 we can find a homogeneous canonical transformation $\chi$ from a conic neighborhood of $\left(x_{0}, \xi_{0}\right)$ to a conic neighborhood of $\left(X_{0}, \Xi_{0}\right) \in T^{*}\left(\mathbf{R}^{n}\right) \backslash 0$ such that $q$ is the pullback of $\Xi_{n-1}+i \Xi_{n}$ by the map $\chi$. As in Proposition 6.1.4 we can then find a corresponding Fourier integral operator with the properties stated there except that $D_{n}$ is replaced by $\partial / \partial \bar{z}$ in (iii), $z=X_{n-1}+i X_{n}$. In fact, the only change is that to satisfy (6.1.12) with $D_{n}$ replaced by $\partial / \partial z$ we have to solve a Cauchy-Riemann equation in each step. This can be done by Cauchy's integral formula. The proof of Theorem 6.1.1 now serves again to deduce Theorem 7.2.1 for the operator $Q$ from the special case of the operator $\partial / \partial \bar{z}$ already established, and we do not repeat the details.

We shall now derive existence theorems from Theorem 7.2.1. In doing so we assume for simplicity that the set $N$ there is equal to the characteristic set $p^{-1}(0)$ although it would be easy to consider a mixture of this case and the one discussed in Chapter VI.

Тнеов м 7.2.4. Assume that $P \in L_{1}^{m}(X)$ has a homogeneous principal symbol $p$, that $\{p, \bar{p}\}=0$ and that $H_{\mathrm{R}_{\theta} p}, H_{\operatorname{Im} p}$ and the cone axis are linearly independent when $p=0$. Let $K$ be a compact subset of $X$ such that no bicharacteristic strip of $P$ stays over $K$. If s is upper semicontinuous in $T^{*}(X) \backslash 0$ and subharmonic in $p^{-1}(0)$, it follows that

$$
u \in \mathscr{E}^{\prime}(K), P u=f, s_{f}^{*} \geqslant s \Rightarrow s_{u}^{*} \geqslant s+m-1 \text {. }
$$

The space

$$
N(K)=\left\{v \in \mathscr{E}^{\prime}(K) ;{ }^{t} P v=0\right\}
$$

is a finite dimensional subspace of $C_{0}^{\infty}(K)$. If $S$ is a lower semi-continuous function in $T^{*}(X) \backslash 0$ which is superharmonic in $p^{-1}(0)$, if $f \in \mathscr{D}^{\prime}(X), s_{f}^{*} \geqslant S$, and $f$ is orthogonal to $N(K)$, then one can find $u \in \mathscr{D}^{\prime}(X)$ with $s_{u}^{*} \geqslant S+m-1$ so that $P u=f$ in a neighborhood of $K$.

Proof. Let $u$ be as in (7.2.1). By the elliptic theory $s_{u}^{*} \geqslant s+m$ in the complement of $p^{-1}(0)$, and by Theorem 7.2.1 we know that $\varphi=\min \left(s_{u}^{*}-s-m+1,0\right)$ is superharmonic in $p^{-1}(0)$. We can now argue as in the proof of $(\mathrm{e}) \Rightarrow(\mathrm{a})$ in Theorem 7.1.5: Let the minimum of $\varphi$ in $p^{-1}(0)$ be taken at $(x, \xi)$. Then $\varphi$ is constant in the bicharacteristic strip through $(x, \xi)$ which by hypothesis contains points over $\mathbf{C} K$. There $s_{u}^{*}=+\infty$ so $\varphi=0$. Hence $\varphi \geqslant 0$ everywhere and (7.2.1) is proved.

The hypotheses on $P$ are also fulfilled by ${ }^{t} P$. Replacing $P$ by ${ }^{t} P$ in (7.2.1) we conclude that $N(K) \subset C_{0}^{\infty}$ and therefore by Fredholm theory that $\operatorname{dim} N(K)<\infty$. 
The existence theorem is now obtained by standard functional analysis as in the proof of Theorem 6.3.1: Let $F=\left\{u \in \mathscr{D}^{\prime}(X), s_{u}^{*} \geqslant S\right\}$ which is a Fréchet space with the topology defined by the seminorms $u \rightarrow\|A u\|_{L_{2}}$ where $A \in L_{1}^{t}(X)$ has a kernel of compact support and $t<S$ in $W F(A)$. The dual space consists of all $v \in \mathscr{E}^{\prime}(X)$ with $s_{v}^{*}>-S$. Since

$$
v \in \mathscr{E}^{\prime}(K),{ }^{t} P v=g, s_{g}^{*}>-S-m+1 \Rightarrow s_{v}^{*}>-S,
$$

the functional analytic arguments at the end of the proof of Theorem 6.3.1 can be applied with $C^{\infty}$ replaced by $F$. The details are left for the reader.

Theorem 7.2.5. Assume that $P \in L_{1}^{m}(X)$ has a homogeneous principal symbol $p$, that $\{p, \bar{p}\}=0$ and that $H_{\operatorname{Re} p}, H_{\operatorname{Im} p}$ and the cone axis are linearly independent when $p=0$. Assume that no bicharacteristic strip stays over a compact subset of $X$. Then we have (a) $\Rightarrow$ (b) $\Rightarrow$ (c) where

(a) For every compact set $K \subset X$ there is another compact set $K^{\prime} \subset X$ such that for each bicharacteristic strip $B$ and each component $C$ of $B \cap \mathbf{C} \pi^{-1}(K)$ which has relatively compact projection in $X$ we have $C \subset \pi^{-1}\left(K^{\prime}\right)$.

(b) For every compact set $K \subset X$ there is another compact set $K^{\prime} \subset X$ such that

$$
v \in \mathscr{E}^{\prime}(X), \text { sing supp }{ }^{t} P v \subset K \Rightarrow \operatorname{sing} \operatorname{supp} v \subset K^{\prime} .
$$

(c) $P$ defines a surjective map from $\mathscr{D}^{\prime}(X)$ to $\mathscr{D}^{\prime}(X) / C^{\infty}(X)$.

Proof. (a) $\Rightarrow$ (b) follows from Corollary 7.2.2, for the projection in $X$ of the wave front set is equal to the singular support. (b) $\Rightarrow$ (c) follows from [46, Theorem 1.2.4], for we can take $K^{\prime}=\varnothing$ when $K=\varnothing$ because no bicharacteristic strip stays over a compact subset of $X$.

After constructing solutions with wave front set in a bicharacteristic strip we shall see in section 7.4 under additional hypotheses concerning $P$ that (c) implies (a). (See Theorem 7.4.2.)

It is easily verified that no bicharacteristic strip stays over a compact subset of $K$ if and only if no leaf of the bicharacteristic foliation in the cosphere bundle stays in a compact set. Secondly condition (a) in Theorem 7.2.5 is equivalent to condition (a) of Theorem 7.1.6 for the bicharacteristic foliation in the cosphere bundle. For this reason the manifold $X$ will be called pseudo-convex with respect to $P$ if condition (a) of Theorem 7.2.5 is fulfilled and $P$ is of principal type in the sense of Definition 6.3.2 which applies with no change in the present situation. 


\subsection{First order differential operators (continued)}

As in section 7.1 we shall consider a homogeneous first order differential operator $L$ in a $C^{\infty}$ manifold $M$ such that $L$ satisfies (7.1.1) and (7.1.2). Before constructing parametrices we have to discuss the properties of the corresponding relation $R \subset M \times M$ consisting of pairs $(x, y)$ of points in the same leaf of the $L$-foliation. The foliation is called regular in $M$ if $R$ is a $C^{\infty}$ submanifold of $M \times M$. (This terminology follows Palais [47]. Haefliger [40] uses the term simple.) When the foliation is regular the quotient topology in the leaf space $M / R$ is Hausdorff if and only if $R$ is closed but in any case regularity implies that every point in $M / R$ is the intersection of a fundamental system of neighborhoods with manifold structure inherited from $M$. Thus $M / R$ is a $C^{\infty}$ manifold when $R$ is a closed $C^{\infty}$ manifold.

In contrast with the real case there is no relation between the condition that $R$ is a $C^{\infty}$ (closed) submanifold of $M \times M$ and the solvability properties of the equation $L u=f$.

Example 7.3.1. Let $L_{0}$ be a real vector field without zeros on a manifold $M_{0}$ and let $L$ be the vector field $L_{0}+i \partial / \partial t$ on $M_{0} \times \mathbf{R}$ where $t$ denotes the variable in $\mathbf{R}$. Then there is global solvability for $L$ since there are no relatively compact leaves for the $L$-foliation and condition (a) of Theorem 7.1.6 is fulfilled with $K=K^{\prime}$ if $K$ is convex in the direction $\partial / \partial t$. The relation $R_{L}$ for $L$ is closed (a manifold) if and only if the relation $R_{L_{0}}$ for $L_{0}$ is. When $L_{0}=\partial / \partial x_{1}$ in $\mathbf{R}^{2} \backslash\{0\}$ it follows that $R_{L}$ is a manifold but not closed and when $L_{0}=\partial / \partial \theta+(r-1) \partial / \partial r$ (with polar coordinates) in $\mathbf{R}^{2} \backslash\{0\}$ it follows that $R_{L}$ is neither closed nor a manifold.

Example 7.3.2. Let $L=\partial / \partial x_{1}+i \partial / \partial x_{2}$ and $M=\mathbf{R}^{3} \backslash\{0\}$. Then $R$ is a closed submanifold of $M \times M$, no leaf of the $L$-foliation is contained in a compact subset of $M$ but we do not have global solvability on $M$.

If $R$ is a closed $C^{\infty}$ submanifold of $M \times M$, then the vector field $(L, 0)$ in $M \times M$ defines a vector field $L^{\prime}$ on $R$. The corresponding relation $R_{L^{\prime}}, R \times R$ is obviously a closed submanifold.

Proposition 7.3.3. The $L^{\prime}$-foliation has no relatively compact leaves if and only if this is true for the L-foliation, and condition (c) in Theorem 7.1.6 is fulfilled by $L^{\prime}$ if and only if it is fulfilled by $L$.

Proof. The first statement is obvious and the second follows immediately if we note that $f(x)+g(y)$ is a $(L, 0)$ subharmonic function of $(x, y) \in M \times M$ if $f$ is $L$ subharmonic, and that $f(x, y(x))$ is $L$ subharmonic if $f(x, y)$ is $L^{\prime}$ subharmonic in $R$ and $y$ is constant as $x$ varies in a leaf. The existence of such $y(x)$ locally over $M / R$ and the existence of partitions of unity in $M / R$ leads to the desired subharmonic functions on $M$. 
We shall now discuss some properties of regular foliations near a compact subset of a leaf. This is required for the construction of solutions with given singularities and will also elucidate the meaning of the regularity condition. (See also Haefliger [40] for a much more thorough discussion.)

At first we consider an arbitrary $k$ dimensional foliation $\Theta$ of an $n$ dimensional manifold $M$. For every $x \in M$ we can choose neighborhoods $V$ such that the restriction of $\Theta$ to $V$ is a fibration with base and fiber diffeomorphic to a ball. The germ $M_{x}^{\prime}$ of the base at the projection of $x$ is independent of the choice of $V$. If $y \in V$ is in the same local leaf as $x$ we obtain a well defined germ of diffeomorphism $\Phi_{y, x}: M_{x}^{\prime} \rightarrow M_{y}^{\prime}$. For every continuous curve $\gamma:[0,1] \in M$ which remains in a leaf we can now define a map $\Phi_{\gamma}: M_{\gamma(0)}^{\prime} \rightarrow M_{\gamma(1)}^{\prime}$, the Poincaré map of $\gamma$, as a composition of maps $\Phi_{\gamma(k / N), \gamma((k-1) / N)}, k=1, \ldots, N$, when $N$ is sufficiently large. The map only depends on the homotopy class of curves $\gamma$ with fixed end points and contained in a leaf of $\Theta$. If $\gamma(0)=x_{0}, \gamma(1)=x_{1}$ the relation $R$ must at $\left(x_{0}, x_{1}\right)$ contain all $(x, y)$ nearby such that $\Phi_{\gamma} x^{\prime}=y^{\prime}$ for the corresponding projections in $M_{x_{0}}^{\prime}$ and $M_{x_{1}}^{\prime}$. These points $(x, y)$ form a germ of manifold of dimension $n-k+2 k=n+k$. Altogether there are at most countably many curves to consider in order to obtain the full relation $R$ at a given point so $R$ must be of dimension $n+k$ if it is a manifold. (This could have been included in the definition of regularity.) Thus regularity means that the relation $R$ near any point $\left(x_{0}, x_{1}\right) \in R$ is obtained as just explained from any choice of a curve $\gamma$ from $x_{0}$ to $x_{1}$ in the leaf of $\Theta$ through these points. This shows that regularity of $\Theta$ in $M$ implies regularity of the restriction to any open subset $M_{1} \subset M$. We shall say that $\Theta$ is semi. regular in $M$ if any compact subset of a leaf has a neighborhood in $M$ where the restriction of $\Theta$ is regular. It is clear that semi-regularity implies that $\Phi_{\gamma}$ only depends on the end points of $\gamma$ or, equivalently, that $\Phi_{\gamma}$ is the identity $M_{x}^{\prime} \rightarrow M_{x}^{\prime}$ for every $\gamma$ in the leaf through $x$ which starts and ends at $x$. We shall prove the converse below. In general the set of all such $\Phi_{\gamma}$ defines a group of germs of diffeomorphisms in $M_{x}^{\prime}$ called the holonomy group of $\Theta$ at $x$. If $y$ is in the same leaf $B$ as $x$, an isomorphism between the holonomy groups at $x$ and at $y$ is given by any curve from $x$ to $y$ in $B$. Up to isomorphism the holonomy group therefore only depends on the leaf so we shall talk about the holonomy group of a leaf also. It is of course a quotient of the fundamental group.

Proposition 7.3.4. Let $B$ be a leaf of the foliation $\Theta$ in $M$ such that the holonomy group of $B$ is trivial. For every compact set $K \subset B$ it is then possible to choose a neighborhood $W$ in $M$ and a diffeomorphism $W \rightarrow U \times T$ which transforms the leaves of $\Theta$ to the fibers of the projection $U \times T \rightarrow T$. In particular, $\Theta$ is regular in $W$.

Proof. Since every compact subset of $B$ has a neighborhood in $M$ which is diffeomorphic 17 - 722909 Acta mathematica 128. Imprimé le 28 Mars 1972. 
to the normal bundle of a neighborhood in $B$, we may assume that $M$ is a real vector bundle with base $B$ and Riemannian structure. If $x \in B$ we denote the fiber at $x$ by $M_{x}$. Let $U$ be a connected relatively compact neighborhood of $K$ in $B$ with homotopy group generated by finitely many closed curves $\gamma_{1}, \ldots, \gamma_{k}$ with base point $x_{0} \in U$. If $\gamma$ is a continuous curve in $B$ the Poincaré map $\Phi_{\gamma}$ is now a map of a neighborhood of 0 in $M_{\gamma(0)}$ to a neighborhood of 0 in $M_{\gamma(1)}$. By hypothesis we can choose a ball $T \subset M_{x_{0}}$ with center at 0 such that $\Phi_{\gamma_{j}}$ is the identity $T \rightarrow T$ for $j=1, \ldots, k$ and $\Phi_{\gamma}$ is defined on $T$ for every curve in $U$ starting at $x_{0}$. If $(x, y) \in U \times T$ and $\gamma$ is a curve in $U$ from $x_{0}$ to $x$ writing $F(x, y)=\Phi_{\gamma} y \in M_{x}$ gives a unique definition of a diffeomorphism from $U \times T$ to a neighborhood of $U$ in $M$ which has the required properties.

In the case of the $L$-foliation the analytic structure in the fibers of $U \times T \rightarrow T$ defined by $L$ might vary with the parameter $t \in T$. However this can always be avoided when $K$ is not an entire leaf. In fact, there are no nontrivial deformations of the analytic structure on a compact subset of a Stein manifold as shown in a recent manuscript by R. S. Hamilton. We shall outline a proof in the case we need:

Proposition 7.3.5. Let $B$ be a leat of the L-foliation in $M$ such that the holonomy group of $B$ is trivial. For every compact set $K \underset{\neq}{\subset} B$ it is then possible to choose a neighborhood $W$ in $M$ and a diffeomorphism $W \rightarrow U \times T$ which transforms $L$ to the form $c\left(L_{U}, 0\right)$ where $L_{U}$ is a first order differential operator in $U$ and $c \in C^{\infty}(U \times T)$.

Proof. By Proposition 7.3.4 we may assume that $M=U \times T$ where $U$ is connected and non-compact, $T$ is a neighborhood of 0 in $\mathbf{R}^{n-2}, B$ is the fiber of $U \times T \rightarrow T$ at 0 and $L$ can be considered as an operator $L_{t}$ in $U$ depending on $t$. If $U^{\prime}$ is a relatively compact neighborhood of $K$ in $U$, which is not compact, we can choose $f_{1}, \ldots, f_{N}$ with $L_{0} f_{j}=0, j=1, \ldots, N$ so that

$$
U^{\prime} \ni x \rightarrow\left(f_{1}(x), \ldots, f_{N}(x)\right)
$$

is an embedding of $\bar{U}^{\prime}$ in $\mathbf{C}^{N}$ as an analytic curve $\Gamma$. This is possible since $U^{\prime}$ lies in a Stein manifold. Now choose $u_{j} \in C^{\infty}(U \times T)$ so that $u_{j}(x, 0)=0$ and

$$
L_{t} u_{j}(x, t)=L_{t} f_{j}(x), \quad x \in U, t \in T .
$$

The existence of $u_{j}$ follows from Theorem 7.1.6 if we first note that

$$
L_{t} t_{j}=\sum t_{p} g_{j v}(x, t), \quad g_{j v} \in C^{\infty}(U \times T),
$$

since $L_{0} f_{j}=0$, and then solve the equation with $g_{j \nu}$ in the right hand side. Write $F_{j}(x, t)=$ $f_{j}(x)-u_{j}(x, t)$. Then 


$$
(x, t) \rightarrow\left(F_{1}(x, t), \ldots, F_{N}(x, t)\right) \in \mathbf{C}^{N}
$$

gives for small $t$ an analytic embedding of $U_{t}^{\prime}=U^{\prime} \times\{t\}$ in $\mathbf{C}^{N}$, close to $\Gamma$. Let us admit for a moment that there exists an analytic retraction $\Phi$ of a neighborhood of $\Gamma$ to $\Gamma$. Then

$$
\left(G_{1}(x, t), \ldots, G_{N}(x, t)\right)=\Phi\left(F_{1}(x, t), \ldots, F_{N}(x, t)\right)
$$

is an $N$-tuple of $C^{\infty}$ functions near $K \times\{0\}$ such that $L_{t} G_{j}=0$ and $G_{j}(x, t)=f_{j}(\varphi(x, t))$ where $\varphi \in C^{\infty}$ and $\varphi(x, 0)=x$. Thus the map $(x, t) \rightarrow(\varphi(x, t), t)$ carries a neighborhood of $K \times\{0\}$ into $U^{\prime \prime} \times T^{\prime}$ and $L$ into $L^{\prime}$ so that $U^{\prime \prime}$ is still a neighborhood of $K$ and $L_{t}^{\prime} f_{j}(x)=0$ for all $x \in U^{\prime \prime}$ and $t \in T^{\prime}$. But then it follows that $L^{\prime}$ has the desired form.

The existence of $\Phi$ follows if we show that that on $\Gamma$ there is an analytic function $P$ with values in $N \times N$ matrices such that $P(z)$ projects $\mathrm{C}^{N}$ on the tangent $T_{z}$ of $\Gamma$ at $z$ for every $z \in \Gamma$. In fact, we can then use the implicit function theorem to show that $\Phi$ is defined in a small neighborhood of $\Gamma$ by $P(\Phi(z))(z-\Phi(z))=0$. Finally, the existence of $P$ is trivial locally. As pointed out to us by M. F. Atiyah the global existence then follows from the fact that the difference between two local choices of $P$ is any analytic section of the analytic vector bundle $W$ on $\Gamma$ such that $W_{z}, z \in \Gamma$, is the set of $N \times N$ matrices mapping $\mathbf{C}^{N}$ into $T_{z}$ and $T_{z}$ to 0 . Indeed, the first cohomology group with values in the sheaf of germs of sections of $W$ is trivial by Theorem B of Cartan (see e.g. [41, Chap. VII). The proof is complete.

In our applications to pseudo-differential operators we want to solve equations of the form $L u=f$ when $u$ and $f$ are in the symbol spaces $S_{\varrho}^{m}$. Let $M$ be a cone manifold, $L$ a complex $C^{\infty}$ vector field on $M$ commuting with multiplication by positive scalars. Denote by $M_{s}$ the quotient of $M$ by the action of $\mathbf{R}_{+}$and let $L_{s}$ be the vector field on $M_{s}$ induced by $L$.

Proposition 7.3.6. The following conditions are equivalent:

(i) For every $f \in C^{\infty}\left(M_{s}\right)$ there exists a solution $u \in C^{\infty}\left(M_{s}\right)$ of $L_{s} u=f$.

(ii) For every $m \in \mathbf{R}$ and every $f \in C^{\infty}(M)$ which is homogeneous of degree $m$ there exists a solution $u \in C^{\infty}(M)$ of $L u=f$ which is homogeneous of degree $m$.

Proof. (ii) is (i) for $m=0$. If $g$ is positive and homogeneous of degree $m$, introducing $u=g u_{1}$ and $f=g f_{1}$ in (ii) reduces $L u=f$ to the form $L u_{1}+c u_{1}=f_{1}$ where $c$, $u_{1}$ and $f_{1}$ are homogeneous of degree 0 . This can be interpreted as an equation $L_{s} u_{1}+c u_{1}=f_{1}$ on $M_{s}$ which has a solution by condition (i). Hence (i) $\Rightarrow$ (ii).

Denote by $S_{\text {hom }}^{m}(M)$ the set of all $a \in S_{1}^{m}(M)$ which are asymptotic sums of homogeneous symbols. If condition (i) in Proposition 7.3.6 is fulfilled it follows that for every $f \in S_{\text {hom }}^{m}(M)$ one can choose $u \in S_{\text {hom }}^{m}(M)$ with $L u-f \in S^{-\infty}$. This simple existence theorem 
would be satisfactory for our purposes but we shall give other existence theorems in the spaces $S_{e}^{m}$ anyway since they follow from a study of the relation defined by $L$ which is indispensible even if we only consider homogeneous symbols.

We shall now examine the relations between various properties of $L$ and of $L_{s}$. In doing so it is convenient to note that there exists a diffeomorphism $M \rightarrow M_{s} \times \mathbf{R}_{+}$commuting with multiplication with positive scalars (acting trivially on $M_{s}$ ). In fact, since $M$ is a fiber bundle with affine structure in the fibers $\mathbf{R}_{+}$, we can construct a global section of $M$ by means of a partition of unity in $M_{s}$. From now on we therefore assume that $M=M_{s} \times \mathbf{R}_{+}$ and denote points in $M$ by $(y, r)$ where $y \in M_{s}$ and $r \in \mathbf{R}_{+}$. Then

where $c \in C^{\infty}\left(M_{s}\right)$.

$$
L=L_{s}+c r \partial / \partial r
$$

Proposition 7.3.7. Condition (7.1.1) is fulfilled by $L$ if and only if it is fulfilled by $L_{s}$ and in addition to $\left[L_{s}, \bar{L}_{s}\right]=\lambda L_{s}-\bar{\lambda} \bar{L}_{s}$ we have

$$
L_{s} \bar{c}-\bar{L}_{s} c=\lambda c-\bar{\lambda} \bar{c} .
$$

Condition (7.1.2) is fulfilled by $L_{s}$ if and only if $\operatorname{Re} L, \operatorname{Im} L$ and the direction of the cone axis are linearly independent. When this is so and we write $L_{s}=a_{s} \vec{\partial}_{s}$ as in (7.1.6), $b=c a_{s}^{-1}$, the condition (7.3.2) reduces to

$$
\partial_{s} b+\bar{\partial}_{s} \bar{b}=0
$$

which means that the real differential form $b+\bar{b}$ is closed in each leat of $L_{s}$.

Proof. Only the equivalence of (7.3.2) and (7.3.2)' requires discussion. We can write (7.3.2) in the form $\left(L_{s}+\bar{\lambda}\right) \bar{c}=\left(\bar{L}_{s}+\lambda\right) c$ and the discussion which led to (7.1.7) shows that $\left(\bar{L}_{s}+\lambda\right) c=a_{s} \bar{a}_{s} \partial_{s} a_{s}^{-1} c$, hence by conjugation $\left(L_{s}+\bar{\lambda}\right) \bar{c}=-\bar{a}_{s} a_{s} \bar{\partial}_{s} \bar{b}$.

It is obvious that (7.3.2) can be violated by a suitable choice of $c$. However, in our applications we will only be interested in the case where $L$ is the Hamilton field $H_{p}$ on the manifold $p=0$ for a function $p$ on the cotangent bundle of some manifold $X$. In this case we have

Proposition 7.3.8. Let $p$ be homogeneous of degree 1 on an open conic subset of $T^{*}(X) \backslash 0$ and suppose that $\operatorname{Re} p, \operatorname{Im} p$ have linearly independent differentials when $p=0$. Let $M$ be the manifold $p=0, L$ the Hamilton field $H_{p}$ on $M$. If the operator $L_{s}$ on $M_{s}$ satisfies (7.1.1) it follows then that $\{\operatorname{Re} p, \operatorname{Im} p\}=0$ when $p=0$, hence that $L$ satisfies (7.1.1).

Proof. The projection of $[L, \bar{L}]$ in $M_{s}$ is equal to $\left[L_{s}, \bar{L}_{s}\right]$ as is immediately seen by letting $[L, \bar{L}]$ operate on functions which are homogeneous of degree 0 . Hence $[L, \bar{L}]=a L-\bar{a} \bar{L}+c \xi$ 
where $\xi$ is the vector field in the direction of the cone axis. Taking the symplectic scalar product with $\xi$ noting that $[L, \bar{L}]=H \quad, \bar{p}\}$ by the Jacobi identity, we obtain $\{p, \bar{p}\}=0$ when $p=0$ by Euler's identity for homogeneous functions.

Assuming that $L$ satisfies (7.1.1) and (7.1.2) we shall now examine when it is possible as in Theorem 6.4.3 to find an isomorphism $M \rightarrow M_{s} \times \mathbf{R}_{+}$such that $L$ becomes equal to $L_{s}$. Obviously this requires that $L_{s}$ satisfies (7.1.2) and also that the projection $\pi_{s}: M \rightarrow M_{s}$ is injective on any leaf $B$ of $L$. The projection $B_{s}=\pi_{s} B$ is then a leaf of $L_{s}$. Using an isomorphism of $M$ and $M_{s} \times \mathbf{R}_{+}$as above we must therefore for any $B_{s}$ by able to find a positive $C^{\infty}$ function $R$ in $B_{s}$ such that $B_{s} \ni y \rightarrow(y, R(y))$ is a leaf of $L$, that is, $L_{s} R=$ $c R$ on $B_{s}$. Writing $R=e^{v}$ this means that $v$ shall be real and that $L_{s} v=c$ or $\vec{\partial}_{s} v=b$ with the notations in Proposition 7.3.7. It follows that $\partial_{s} v=\bar{b}$ or equivalently that $d v=\bar{b}+b$ where $d$ is the exterior differential in $B_{s}$. Thus we have proved

Proposition 7.3.9. Assume that $L$ satisfies (7.1.1) and that $L_{s}$ satisfies (7.1.2). If $B$ is a leaf of $L$ it follows that the projection $B \rightarrow B_{s}=\pi_{s} B$ is injective if and only if the closed differential form $\bar{b}+b$ in Proposition 7.3 .8 is exact in $B_{s}$.

If there exists a properly embedded leaf $B_{s}$ with trivial holonomy group and $H^{1}\left(B_{s}, \mathbf{R}\right) \neq 0$, then one can find $c \in C^{\infty}\left(M_{s}\right)$ so that the projection $B \rightarrow B_{s}$ is not injective although (7.3.2) is valid near $B_{s}$. However, for regular Hamilton fields this situation can not occur:

Proposition 7.3.10. Let $p$ be homogeneous of degree 1 on an open conic subset of $T^{*}(X) \backslash 0$, and suppose that $H_{\mathrm{Re} p}, H_{\operatorname{Im} p}$ and the cone axis are linearly independent when $p=0$. Let $M$ be the manifold $p=0$ and $L$ the Hamilton field $H_{p}$ on $M$. If $L_{s}$ satisfies (7.1.1) and the $L_{s}$ foliation is semi-regular, it follows that the projection $\pi_{s}: M \rightarrow M_{s}$ is injective on the leaves of the L-foliation, and the L-foliation is semi-regular too.

Note that by Proposition 7.3.8 it follows that $L$ satisfies (7.1.1) so the $L$-foliation is defined.

Proof. Let $B$ be a leaf of $L$ and $\gamma$ a curve in $B$ starting at $\left(x_{0}, \xi_{0}\right)$ such that $\pi_{s} \gamma$ is closed in $M_{s}$. We shall prove that $\gamma$ is closed in $M$ also. To do so we note that the local leaf spaces $M_{(x, \xi)}^{\prime}$ introduced above are symplectic manifolds with symplectic form inherited from the one in $T^{*}(X) \backslash 0$. This follows from the fact that $H_{\mathrm{Re} p}$ and $H_{\operatorname{Im} p}$ are orthogonal to the tangent space of $M$. (See also sections 3.3 and 6.6.) The Poincaré map $\Phi_{\gamma}$ is canonical since this is obviously true for curves which stay in a sufficiently small neighborhood of a point. But the fact that the $L_{s}$-foliation is semi-regular shows that $\Phi_{\gamma}$ must project to the identity in $M_{s}$ so $\Phi_{\gamma}(x, \xi)=(x, \tau(x, \xi) \xi)$ in a neighborhood of $\left(x_{0}, \xi_{0}\right), \tau>0$. Thus $\Phi_{\gamma}$ multi- 
plies the symplectic volume element by $\tau^{n-2}$ which implies that $\tau=1$ and that $\Phi_{\gamma}$ is the identity. The proposition is proved.

Proposition 7.3.11. Let $M$ be a cone manifold, $L$ an operator commuting with multiplication by positive scalars such that $L$ satisfies (7.1.1), $L_{s}$ satisfies (7.1.2) and the $L_{\text {s }}$ foliation in $M_{s}$ is regular with separated leaf space. Then there is a diffeomorphism $M \rightarrow M_{s} \times \mathbf{R}_{+}$commuting with scalars which transforms $L$ to $L_{s}$ if (and only if) the projection $\pi_{s}: M \rightarrow M_{s}$ is injective on the leaves of $L$.

Proof. The problem is to find a section $M_{s} \rightarrow M$ consisting of leaves of the $L$-foliation. If we choose a diffeomorphism $M \rightarrow M_{s} \times \mathbf{R}_{+}$and the corresponding representation (7.3.1) of $L$, the proof of Proposition 7.3.9 shows that this is equivalent to the existence of a real solution $v \in C^{\infty}\left(M_{s}\right)$ of the equation $L_{s} v=c$, and Proposition 7.3.9 shows that this equation has a unique solution in each leaf apart from an additive constant. If we choose $v=0$ on a manifold transversal to a leaf of the $L_{s}$-foliation we obtain a $C^{\infty}$ solution $v$ in the union of all leaves in a neighborhood. By means of a partition of unity in the leaf space $M_{s} / R_{s}$ we can piece these solutions together to a solution in $M_{s}$.

Note that Proposition 7.3.10 shows that Proposition 7.3.11 is applicable in the Hamiltonian case. This is the case which will occur in the construction of parametrices.

When $L$ can be put in the form of Proposition 7.3.11 the equation $L u=f$ can be solved for symbols $u$ and $f$ by regarding them as functions on $M_{s}$ depending on the parameter $r \in \mathbf{R}_{+}$. To do so we must only show that there is a continuous linear way of choosing solutions of the equation $L_{s} u=f$. For the sake of completeness we discuss this question in the next three propositions although it is not essential later. In the first two propositions we write $L$ instead of $L_{s}$.

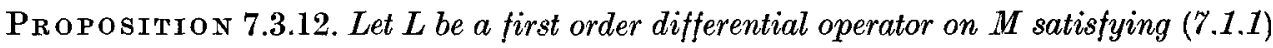
and (7.1.2), and assume that the $L$-foliation is regular. Let $K$ be a compact subset of $M$ such that no leaf of the $L$-foliation is contained in $K$. Then there exists a mapping $E: C_{0}(K) \rightarrow C(M)$ such that

(i) $E$ is continuous from $C_{0}^{k}(K)$ to $C^{k}(M)$ for $k=0,1,2, \ldots$

(ii) $E$ commutes with multiplication by $C^{\infty}$ functions which are constant on the leaves of the L-foliation in a neighborhood $V$ of $K$.

(iii) $L E f=f$ for all $f \in C_{0}(K)$.

Proof. First note that the regularity of the $L$-foliation implies that $K \cap B$ is compact for every leaf $B$. In fact, if $x \in K \cap \bar{B}$ (closure of $B$ in $M$ ) and if $V$ is a neighborhood of $x$ 
such that $R$ is a manifold in $V \times V$, then $B \cap V$ is closed in $V$ so $x \in B$ and the $B$ topology and $M$-topology agree on $B \cap V$.

By Proposition 7.3.5 it suffices to consider the case where $M=U \times T$ and $U$ is of dimension 2 with $L$ tangent to the fibers of $U \times T \rightarrow T$. For using a partition of unity near $K$ consisting of functions constant on the leaves of the foliation near $K$ we can pass to the general case. Clearly it suffices to consider the case $M=U$ and the statement is very well known then. (A direct proof is obtained by taking a positive density in $U$ and writing $E$ locally by means of Cauchy's integral formula in a system of local coordinates. Piecing together one obtains $E_{1}$ with the desired properties except that

$$
L E_{1} f=f+R f, \quad R f(x)=\int R(x, y) f(y) d y, \quad f \in C_{0}^{\infty}(K) .
$$

Here $R \in C^{\infty}$ so we can choose $S$ so that $L_{x} S(x, y)=R(x, y)$ in a neighborhood of $K \times K$. Thus $E f=E_{1} f-S f$ has the required properties.)

Proposition 7.3.13. Let $L$ be a first order differential operator on $M$ satisfying (7.1.1) and (7.1.2), and assume that the $L$-foliation in $M$ is regular with separated leaf space (i.e. the relation $R$ is a closed submanifold of $M \times M$ ). In addition assume that $M$ is pseudo-convex with respect to $L$. Then there exists a mapping $E: C_{0}(M) \rightarrow C(M)$ such that

(i) $E$ is continuous $C_{0}^{k}(M) \rightarrow C^{k}(M)$ for $k=0,1,2, \ldots$

(ii) E commutes with multiplication by functions which are constant on the leaves of the L-foliation.

(iii) $L E f=f$ for all $f \in C_{0}(M)$.

Proof. By piecing together operators given by Proposition 7.3.12 we obtain all the required properties except that $L E f=f+S f$ where $S$ is an integral operator along the leaves of the foliation with $C^{\infty}$ kernel, defined on $R$. Using the existence theorem for the operator $L^{\prime}$ induced by $L$ on $R$ (see Proposition 7.3.3) we can eliminate the error term $S$.

Proposition 7.3.14. Let L satisfy the conditions in Proposition 7.3.12 (resp.7.3.13) and set $M^{\prime}=M \times \mathbf{R}_{+}$. If $f \in S_{Q}^{m}\left(M^{\prime}\right)$ and supp $f \subset K \times \mathbf{R}_{+}$(resp. supp $f$ has a compact projection in $M)$ then $u(x, r)=E_{x} f(x, r)$ is also in $S_{e}^{m}(M)$ and $L u=f$ in $K \times \mathbf{R}_{+}\left(\right.$in $\left.M^{\prime}\right)$. Moreover, the map $f \rightarrow u$ commutes with multiplication by functions which are constant on the leaves of the $L$-foliation in $M^{\prime}$.

Proof. That $f \in S_{e}^{m}$ means that for every semi-norm $q$ in $C^{k}(M)$ and every $j \geqslant 0$ we have

$$
q\left(\partial^{i} f / \partial r^{j}\right)=O\left(r^{m-\varrho j+(1-e) k}\right)
$$

as $r \rightarrow \infty$. Application of $E$ does not affect this condition in view of the continuity $C_{0}^{k} \rightarrow C^{k}$. 
As a preparation for the constructions in section 7.4 we shall now prove results similar to Proposition 7.3.14 where $m$ varies but is a subharmonic function. The first case to consider is the $\bar{\partial}$ operator on a Riemann surface where we can use well known Carleman estimates.

LEMMA 7.3.15. Let $B$ be a $C^{\infty}$ compact connected Riemann surface with nonempty boundary and let $\varphi \in C^{\infty}(B)$ be strictly subharmonic. Fix a Hermitian metric in $B$. If $f$ is $a$ square integrable $(0,1)$ form in $B$, the equation $\bar{\partial} u=f$ has a unique solution $u=E(\tau) f$ such that

$$
\|u\|_{\tau}^{2}=\int|u|^{2} e^{-2 \tau \varphi} d b
$$

is minimal, db denoting the Riemannian volume element. Then $E(\tau)$ is a $C^{\infty}$ function of $\tau$ and for the operator norm ||$_{\tau}$ corresponding to the $L^{2}$ norms with weights $e^{-2 \tau \varphi}$ we have

$$
\left|E^{(k)}(\tau)\right|_{\tau} \leqslant C_{k} \tau^{-\frac{1}{2}}, \tau>1, k=0,1,2, \ldots
$$

Proof. The existence of $u$ and the bound (7.3.3) for $k=0$ can be found in Hörmander [41] even in the case of Stein manifolds. The proof consists in noting that in a local coordinate system the Hilbert space adjoint of $\partial / \partial \bar{z}$ is $L_{\tau} v=-\partial v / \partial z+2 \tau \partial \varphi / \partial z v+c v$ where $c$ is independent of $\tau$. An integration by parts then gives when $v \in C_{0}^{\infty}$ (int $B$ )

$$
\int\left|L_{\tau} v\right|^{2} e^{-2 \tau \varphi} d b=\int|\bar{\partial} v|^{2} e^{-2 \tau \varphi} d b+\frac{1}{2} \tau \int(\Delta \varphi)|v|^{2} e^{-2 \tau \varphi} d b+\ldots
$$

where dots indicate terms which can be estimated by $\|v\|$ and $\|\bar{\partial} v\|$. This leads to $\tau\|v\|_{\tau}^{2} \leqslant C\left\|L_{\tau} v\right\|_{\tau}^{2}, v \in C_{0}^{\infty}$ (int $B$ ), and by duality the assertion follows. For the details we refer to Hörmander [41].

If we write $u_{\tau}=E(\tau) f$ the definition means that $\bar{\partial} u_{\tau}=f$ and that

$$
\int u_{\tau} \bar{v} e^{-2 \tau \varphi} d b=0, v \in A
$$

where $A$ is the space of square integrable analytic functions in $B$. In particular we can take $v=u_{\tau}-u_{\tau+h}$ and obtain

$$
\int\left|u_{\tau}-u_{\tau+h}\right|^{2} e^{-2 \tau \varphi} d b=\int u_{\tau+h} \overline{\left(u_{\tau}-u_{\tau+h}\right)} e^{-2 \tau \varphi}\left(e^{-2 h \varphi}-1\right) d b .
$$

By Cauchy-Schwarz' inequality this gives

$$
\int\left|u_{\tau}-u_{\tau+h}\right|^{2} e^{-2 \tau \varphi} d b \leqslant \int\left|u_{\tau+h}\right|^{2} e^{-2 \tau \varphi}\left|e^{-2 h \varphi}-1\right|^{2} d b .
$$


Thus $\left(u_{\tau+h}-u_{\tau}\right) / h$ is bounded in $L^{2}$ norm as $h \rightarrow 0$. If $w$ is any weak limit, then it follows from (7.3.4) that

$$
\int w \bar{v} e^{-2 \tau \varphi} d b=\int u_{\tau} \bar{v} e^{-2 \tau \varphi} 2 \varphi d b, v \in A
$$

and since $w \in A$ this shows that $w$ is unique so that $E(\tau)$ is weakly differentiable. The preceding formula can be written

$$
w-2 \varphi u_{\tau}=E(\tau)\left(\bar{\partial}\left(w-2 \varphi u_{\tau}\right)\right)=-2 E(\tau)\left(u_{\tau} \bar{\partial} \varphi+\varphi f\right)
$$

which leads to

$$
E^{\prime}(\tau) f=2 \varphi E(\tau) f-2 E(\tau)(\vec{\partial} \varphi E(\tau) f+\varphi f)
$$

It follows from this that $E$ is infinitely differentiable and, by induction with respect to $k$, that (7.3.3) is valid.

When applying the lemma we shall have to pass from $L^{2}$ norms to maximum norms. To do so we note first that if $u$ is defined in $D_{r}=\{z ; z \in \mathbf{C},|z|<r\}$ then

$$
\left|D^{\alpha} u(0)\right| \leqslant C_{\alpha}\left(r \sup _{D_{r}}\left|D^{\alpha} \partial u / \partial \bar{z}\right|+r^{-1-|\alpha|}\|u\|_{L^{2}\left(D_{r}\right)}\right)
$$

In fact, it suffices to prove this when $r=1$ and then it follows immediately by using a parametrix for $\partial / \partial \bar{z}$. If $\varphi \in C^{1}$ is real valued with given bounds for the first order derivatives it follows that for $\tau>1$

$$
\left|D^{\alpha} u(0)\right| e^{-\tau \varphi(0)} \leqslant C_{\alpha}\left(r \sup _{D_{r}}\left|D^{\alpha} \partial u / \partial \bar{z}\right| e^{-\tau \varphi(z)}+r^{-1-|\alpha|}\left\|u e^{-\tau \varphi}\right\|_{L^{z}(D r)}\right)
$$

if $r \tau \leqslant 1$. In fact, $\varphi(z)=\varphi(0)+O(|z|)$.

Now take $u=E^{(k)} f$ and let $K$ be a compact subset of $B$ which does not meet the boundary. Then we have $\partial u / \partial \bar{z}=f$ for $k=0$ and $\partial u / \partial \bar{z}=0$ for $k>0$. Hence by (7.3.3), (7.3.4)

$$
\sup _{\bar{K}}\left|E^{(k)}(\tau) f\right|_{j} e^{-\tau \varphi} \leqslant C_{k j}\left(\tau^{-1} \sup _{B}|f|_{j} e^{-\tau \varphi}+\tau^{j+\frac{1}{2}} \sup _{B}|f|_{0} e^{-\tau \varphi}\right), \tau>1
$$

where $\|$, now stands for the sum of the derivatives of order $\leqslant j$ computed in terms of some system of local coordinates.

The notation $S_{e}^{m}$ introduced in section 1.1 is also applicable when $M$ is a cone manifold and $m$ is a function on $M$ which is homogeneous of degree 0 . If $a$ is a positive function on $M$ which is homogeneous of degree 1 , then $a^{m} \in S_{\varrho}^{m}$ for $\frac{1}{2}<\varrho<1$. In fact, the derivatives of order $\alpha$ will behave as if $a^{m}$ were in $S_{1}^{m}$ apart from a factor $(\log a)^{|\alpha|}$. (Symbols with this behavior were considered by Unterberger-Bokobza [51] before symbols of type $\varrho, \delta$ were introduced.) 
Proposition 7.3.16. Let $M^{\prime}=T \times B \times \mathbf{R}_{+}$where $T$ is an open set in $\mathbf{R}^{v}$, and $B$ is a twodimensional connected non-compact manifold with a complex vector field $L$ satisfying (7.1.1), (7.1.2), which we regard as a vector field on $M^{\prime}$. Let $\varphi \in C^{\infty}(B)$ be a strictly subharmonic function which we also regard as a function on $M^{\prime}$. If $f \in S_{Q}^{\varphi}\left(M^{\prime}\right), \frac{1}{2}<\varrho<1$, and if $f$ vanishes except over a compact subset $K$ of $B$ then one can find $u \in S_{Q}^{\varphi}\left(M^{\prime}\right)$ with $L u=f$ over a neighborhood of $K$.

Proof. We denote the variable in $T$ by $t$, in $B$ by $z$ and in $\mathbf{R}_{+}$by $e^{\tau}$. The hypothesis is that over any compact set in $T \times B$ for $\tau>1$

$$
|Q f| \leqslant C e^{\tau(\varphi+k(1-\varrho))}
$$

if $Q$ is a differential operator in $M^{\prime}$ of order $k$ which is translation invariant in $\tau$. With $E(\tau)$ defined by Lemma 7.3.15 with $B$ replaced by a suitable neighborhood of $K$ we set

$$
u(t, z, \tau)=E(\tau) f(t, z, \tau) .
$$

The statement now follows immediately from (7.3.6).

\subsection{Solutions with prescribed singularities}

Recall that in section 7.2 we introduced the subset $N$ of $p^{-1}(0)$ where (7.0.1) is valid and $H_{\text {Re } p}, H_{\operatorname{Im} p}$ are linearly independent of the cone axis. The bicharacteristic foliation defined by $H_{p}$ in $N$ gives rise to a foliation in the image $N_{s}$ of $N$ in the sphere bundle. In fact, $H_{p}$ can be regarded as a vector field in $N_{\mathrm{s}}$ if the degree $m$ of $p$ is one, and in general the Hamilton field of $q p$ where $q$ is of degree 1-m defines a foliation independent of the choice of $q$.

THEOREM 7.4.1. Let $\Omega$ be an open connected non-compact subset of a leat $B_{3}$ of the bicharacteristic foliation in $N_{s}$ and assume that the holonomy group of $\Omega$ is trivial. Let $\Gamma$ be the closed cone in $T^{*}(X) \backslash 0$ generated by $\Omega$ and $\Gamma^{\prime}$ the intersection of the closed cones generated by $\Omega \backslash \Omega_{0}$ when $\Omega_{0}$ is compact in $\Omega$. Let $s$ be a lower semi-continuous function in $T^{*}(X) \backslash 0$ which is homogeneous of degree $0,+\infty$ outside $\Gamma$ and has a superharmonic restriction to $\Omega \backslash \Gamma^{\prime}$. The one can find $u \in \mathscr{D}^{\prime}(X)$ with

$$
W F(P u) \subset \Gamma^{\prime}, \quad s_{u}^{*} \geqslant s \text { in } \Gamma^{\prime}, \quad s_{u}^{*}=s \text { in } \mathrm{C}^{\prime} .
$$

In particular, $W F(u) \backslash \Gamma^{\prime}=\Gamma \backslash \Gamma^{\prime}$.

Proof. We may assume that the order of $P$ is one. As in the proof of Theorem 6.2.1 we shall begin by proving a weaker result where $\Omega$ is replaced by a compact subset $K, s$ is a $C^{\infty}$ strictly superharmonic function in $\Omega$ and $\Gamma, \Gamma^{\prime}$ are replaced by conic neighborhoods 
of $K$ and $\partial^{\prime} K$. First note that by Proposition 7.3.5 we can choose a connected neighborhood $U$ of $K$ in $B_{s}$, a neighborhood $T_{s}$ of 0 in $\mathbf{R}^{2 n-5}$ and a diffeomorphism

$$
U \times T_{s} \rightarrow W_{s}
$$

where $W_{s}$ is a neighborhood of $U$ in $N_{s}$ such that $U \times\{0\}$ is mapped to $U$ and if $L=H_{p}$ then $L_{s}$ restricted to $W_{s}$ and pulled back to $U \times T_{s}$ is proportional to the operator $L_{U}$ in $U$ lifted to $U \times T_{s}$. In particular, the $L_{s}$-foliation is regular in $W_{s}$ so if $W=\pi_{s}^{-1} W_{s}\left(\pi_{s}\right.$ is the projection $\left.T^{*}(X) \backslash 0 \rightarrow S^{*}(X)\right)$ it follows from Propositions 7.3.10 and 7.3.11 that there is a diffeomorphism

$$
U \times T_{s} \times \mathbf{R}_{+} \rightarrow W
$$

commuting with multiplication by positive scalars such that $H_{p}$ restricted to $W$ is also proportional to $L_{U}$.

As in the discussion of Poincaré maps in section 7.3 it is clear that $T=T_{s} \times \mathbf{R}_{+}$is a conic symplectic manifold of dimension $2(n-2)$. Choose a conic Lagrangean manifold $\Lambda_{T} \subset T$ containing $0 \times \mathbf{R}_{+}$and a closed conic neighborhood $\Lambda_{T}^{\prime} \subset \Lambda_{T}$ of $0 \times \mathbf{R}_{+}$. Then the image $\Lambda$ of $U \times \Lambda_{T}$ in $W$ is a Lagrangean manifold and $U \times \Lambda_{T}^{\prime}$ maps to a closed subset containing $K$. Corresponding to the decomposition $T=T_{s} \times \mathbf{R}_{+}$we have a decomposition $\Lambda_{T}=\Lambda_{T_{s}} \times \mathbf{R}_{+}$such that $H_{p}$ acts along $\Lambda_{T_{s}}$.

Our purpose is to use a local version of Theorem 5.3.2 with $\frac{2}{3}<\varrho<1$ and $m^{\prime}$ replaced by $-s-n / 4$. In view of Proposition 7.3.16 it is clear that Theorem 5.3.2 remains valid although $m^{\prime}$ is not a constant. What remains is to discuss the choice of a principal symbol. First we choose a non-vanishing section $\omega$ of $L \otimes \Omega_{\frac{1}{2}}$ in $\Lambda$ which is homogeneous of degree $-n / 4$ and satisfies the equation $i^{-1} \mathscr{L}_{H_{p}} \omega+c \omega=0$. This is obviously possible since $L \otimes \Omega_{\underline{z}}$ is a trivial line bundle and we have existence theorems for $H_{p}$ acting on symbols of degree 0 . In Theorem 5.3.2 we shall take $a=u \omega$ where the pullback $v$ of $u$ to $U \times \Lambda_{T}$ vanishes outside $K^{\prime} \times \Lambda_{T}^{\prime}, K^{\prime}$ a compact neighborhood of $K$ in $U, L_{U} v=0$ in a neighborhood of $K \times \Lambda_{T}^{\prime}$, $v \in S^{-s}\left(U \times \Lambda_{T}\right)$ and $v^{-1} \in S^{s}$ in a conic neighborhood of $\left\{z_{0}\right\} \times\{0\} \times \mathbf{R}_{+}, \frac{1}{2}<\varrho<1$. Here $z_{0}$ is any given point in $K$. To construct such a function $v$ we first note that if $s\left(z, z_{0}\right)$ is the analytic part of the Taylor expansion of $s$ at $z_{0}$ to the second order, then $s\left(z_{0}, z_{0}\right)=s\left(z_{0}\right)$ and $s(z)=\operatorname{Re} s\left(z, z_{0}\right)-e\left(z, z_{0}\right)$, where $e\left(z, z_{0}\right) \geqslant b\left|z-z_{0}\right|^{2}$ in a neighborhood $O$ of $z_{0}$ for some positive constant $b$. Here we are working with local analytic coordinates at $z_{0}$. Let $\chi \in C_{0}^{\infty}(O)$ be equal to 1 near $z_{0}$ and set

$$
v(z, t, r)=\chi(z) r^{-s\left(z, z_{0}\right)}-w(z, t, r),(z, t, r) \in U \times \Lambda_{r_{s}}^{\prime} \times \mathbf{R}_{+}
$$

The function $w$ shall satisfy the equation 


$$
L_{U} w(z, t, r)=\left(L_{U} \chi\right) r^{-s\left(z, z_{0}\right)}=f
$$

in a neighborhood of $K \times \Lambda_{T_{s}}^{\prime} \times \mathbf{R}_{+}$. Since for some $\varepsilon>0$ we have $\operatorname{Re} s\left(z, z_{0}\right)>s(z)+\varepsilon$ in supp $\vec{\partial} \chi$ it follows that $f \in S_{\varrho}^{-s-\varepsilon}$. By Proposition 7.3.16 we conclude that $w$ can be found in $\mathcal{S}_{Q}^{-s-\varepsilon}$.

Summing up, by applying Theorem 5.3.2 with the principal symbol just constructed we obtain for $\frac{2}{3}<\varrho<1$ an element $u \in I_{e}^{-s-n / 4}\left(X, K^{\prime} \times \Lambda_{T}^{\prime}\right)$, thus $s_{u}^{*} \geqslant s$, such that $P u \in$ $\left.I_{\ell}^{-s+1-e-n / 4}\left(X, \overline{\left(K^{\prime} \backslash K\right.}\right) \times \Lambda_{T}^{\prime}\right)$ and $s_{u}^{*}=s$ at a given point in $K$. (Here we have of course used Theorem 5.4.1.)

To finish the proof we can repeat the functional analytic arguments in Lemma 6.2.2. Briefly the argument is as follows: The definition of the Fréchet space $F$ there is modified to be the set of all $u \in \mathscr{D}^{\prime}(X)$ such that $s_{u}^{*} \geqslant s$ and $W F(P u) \subset \Gamma^{\prime}$. If $T$ is a pseudo-differential operator of order $s\left(z_{0}\right)<\infty$ which is not characteristic at $z_{0} \in \Omega \backslash \Gamma^{\prime}$, it follows from the preceding construction that.

$$
\left\{u \in F ; T u \in L_{\mathrm{loc}}^{2}\right\}
$$

is of the first category in $F$. For the proof it is important to note that on every compact subset of $\Omega \backslash \Gamma^{\prime}$ the function $s$ is the supremum of a family of $C^{\infty}$ strictly superharmonic functions. The proof is now completed by the same arguments as in the proof of Theorem 6.2 .1 .

Remark. The proof of the preceding theorem would have been considerably simplified if $s$ had been assumed to be harmonic. In fact, it would not have been necessary to prove Proposition 7.3.16 then. However, since combination of Theorems 7.2.1 and 7.4.1 show that superharmonicity is exactly the right condition it seems worth while to accept these complications although they are not required in our applications.

We can now prove the equivalence of the conditions in Theorem 7.2.5 when the bicharacteristic foliation is semi-regular and the leaves are properly embedded. Note that even in the case of first order differential operators we needed such hypotheses in section 7.1 .

THEOREM 7.4.2. Assume that $P \in L_{1}^{m}(X)$ has a homogeneous principal symbol $p$, that $\{p, \bar{p}\}=0$ and that $H_{\mathrm{Re} p}, H_{\operatorname{Im} p}$ and the cone axis are linearly independent when $p=0$. Assume further that no bicharacteristic strip stays over a compact subset of $X$ and that the bicharacteristic foliation is semi-regular. If $P$ defines a surjective map from $\mathscr{D}^{\prime}(X)$ to $\mathscr{D}^{\prime}(X) / C^{\infty}(X)$ it follows then that for every compact set $K \subset X$ there is another compact set $K^{\prime} \subset X$ such that for each bicharacteristic strip $B$ and each component $C$ of $B \cap \mathbf{C} \pi^{-1}(K)$ which is relatively compact in $B$ we have $\pi C \subset K^{\prime}$.

Proof. Assume that for some $K$ no such $K^{\prime}$ exists. Choose sequences of leaves $B_{j}$, 
components $C$, of $B_{j} \cap \mathbf{C} \pi^{-1}(K)$ such that $C_{j}$ is relatively compact in $B$, and points $\left(x_{j}, \xi_{j}\right) \in C_{j}$ such that $x_{j} \rightarrow \infty$ in $X$. We shall then construct $f \in \mathscr{D}^{\prime}(X)$ such that $P u-f$ is not in $C^{\infty}$ for any distribution $u$. In doing so we may assume that the cones generated by $C$, are disjoint.

Choose a compact neighborhood $K_{1}$ of $K$ in $X$ and let $C_{j}^{\prime}$ be $C_{j}$ with such a small neighborhood of $\left(x_{j}, \xi_{j}\right)$ removed that $\pi\left(\partial^{\prime} C_{j}^{\prime} \cap \pi^{-1}(\mathcal{C} K)\right) \rightarrow \infty$ as $j \rightarrow \infty$. In $C_{j}^{\prime}$ we can find a harmonic function $s_{j}$ which is 0 on the part of the boundary which lies over $K$ but is so large negative on the part of the boundary near $\left(x_{j}, \xi_{j}\right)$ that $s_{j}<-j$ at some point in $C_{j}$ which lies over $K_{1}$. Let $\Gamma_{j}$ be the closed cone generated by $C_{j}^{\prime}$, let $\Gamma_{j}^{\prime}$ be generated by the part of the boundary near $\left(x_{j}, \xi_{j}\right)$ and $\Gamma_{j}^{\prime \prime}$ be generated by the boundary over $K$. In view of Theorem 5.4.1 we can choose $u_{j} \in \mathscr{D}^{\prime}(X)$ corresponding to the function $s_{j}$, which gives

$$
\begin{gathered}
W F\left(u_{j}\right) \subset \Gamma_{j}, \quad s_{u_{j}}^{*} \geqslant 0 \text { over } K, \quad \inf _{K_{1}} s_{u_{j}}^{*}<-j, \quad P u_{j}=f_{j}+g_{j}, \\
W F\left(f_{j}\right) \subset \Gamma_{j}^{\prime}, \quad W F\left(g_{j}\right) \subset \Gamma_{j}^{\prime \prime} .
\end{gathered}
$$

The support of $f$, can be chosen so close to sing supp $f_{f}$ that the supports of the terms in the sum

are locally finite.

$$
f=\sum f_{j}
$$

Assume now that for some $u \in \mathscr{D}^{\prime}(X)$ we have $P u-f \in C^{\infty}$. Let $u \in H_{(s)}$ in $K_{1}, s \leqslant 0$. Then $W F\left(P\left(u-u_{j}\right)\right)$ does not meet $C_{j}$ and $u-u_{j} \in H_{(s)}$ at the boundary of $C_{j}$. If $V_{j}$ is the closed cone generated by $C_{j}$ and $A$ is a pseudo-differential operator with $W F(A)$ close to $V$, and $W F(I-A) \cap V_{j}=\varnothing$ it follows from Theorem 7.2.4 applied to $A\left(u-u_{j}\right)$ that $u-u_{j} \in H_{(s)}$ at every point in $C_{j}$. But since $u_{j}$ is not in $H_{(-j)}$ at some such point over $K_{1}$ whereas $u \in H_{(s)}$ there, we obtain a contradiction when $j>-s$. The proof is complete.

\subsection{Construction of a parametrix}

Let $P$ as usual be a properly supported operator $\epsilon L_{1}^{m}(X)$ with homogeneous principal symbol $p$ such that $\{p, \bar{p}\}=0$ and $H_{\mathrm{Re} p}, H_{\operatorname{Im} p}$ are linearly independent of the cone axis in $N=p^{-1}(0)$. Moreover, we shall assume that the bicharacteristic foliation in the image $N_{s}$ of $N$ in the cosphere bundle is regular with separated leaf space. Assuming for a moment that $m=1$ we obtain from Propositions 7.3.10 and 7.3.11 a diffeomorphism $N_{s} \times \mathbf{R}_{+} \rightarrow N$ commuting with the action of $\mathbf{R}_{+}$such that $L=H_{p}$ is just the operator $L_{s}$ in $N_{s}$. Since the relation $C_{s}$ defined by the $L_{s}$ foliation is by hypothesis a closed submanifold of $N_{s} \times N_{s}$ and the bicharacteristic relation $C \subset N \times N$ is identified with $N_{s} \times N_{s} \times \Delta_{\mathbf{R}_{+}}$we conclude that $C$ is a closed submanifold of $T^{*}(X \times X) \backslash 0$. The proof of Proposition 6.5.2 
shows that $C$ is in fact a homogeneous canonical relation. Naturally this is also true if the order $m$ is not equal to 1 for multiplication of $P$ by an elliptic factor does not change $C$.

In the following theorem we assume for the sake of simplicity that $P \in L_{\mathrm{hom}}^{m}(X)$, that is, that the symbol is an asymptotic sum of homogeneous functions all of which are of degree $\leqslant m-1$ except $p$.

TH Е О Е $\mathrm{M}$ 7.5.1. Let $P \in L_{\mathrm{hom}}^{m}(X)$ be properly supported and have a homogeneous principal symbol $p$ such that $\{p, \bar{p}\}=0$ and $H_{\mathrm{R} \theta p}, H_{\mathrm{Im} p}$ are linearly independent of the cone axis in $N=p^{-1}(0)$. F'urther assume that the bicharacteristic foliation in $N_{s}$ is regular with separated leaf space and that $X$ is pseudo-convex with respect to $P$. Then there exists a linear operator $E: C_{0}^{\infty}(X) \rightarrow C^{\infty}(X)$ such that

(a) $P E=I+R$ where $R$ is an integral operator with $C^{\infty}$ kernel.

(b) $W F^{\prime}(E) \subset \Delta^{*} \cup C$ and $E \in I_{\text {hom }}^{-m}\left(X \times X, C^{\prime}\right)$ outside $\Delta^{*}$.

(c) $E$ is continuous from $H_{(s)}^{\text {comp }}(X)$ to $H_{(s+m-1)}(X)$ for every $s \in \mathbf{R}$.

Proof. For the operator $\partial / \partial \bar{z}$ in $\mathbf{R}^{n}, z=x_{n-1}+i x_{n}$ the fundamental solution

$$
E v(x)=\pi^{-1} \int v\left(x^{\prime}, x^{\prime \prime}-y^{\prime \prime}\right)\left(y_{n-1}+i y_{n}\right)^{-1} d y^{\prime \prime}, v \in C_{0}^{\infty}\left(\mathbf{R}^{n}\right)
$$

has the preceding properties since $C^{\prime}$ is then the normal bundle of $\left\{(x, y) \in \mathbf{R}^{n} \times \mathbf{R}^{n}, x^{\prime}=y^{\prime}\right\}$. As in the proof of Theorem 7.2.1 we can use Lemma 7.2.3 and Propositions 6.1.3, 6.1.4 to construct $E$ locally near a point in $\Delta^{*}$. Piecing together local contributions exactly as in the proof of Theorem 6.5.3 we obtain $E$ with all the stated properties except that (a) is weakened to

$$
P E-I=R \in I_{\mathrm{hom}}^{-1}\left(X \times X, C^{\prime}\right) .
$$

We now apply Theorem 5.3.2 where the hypotheses are fulfilled for homogeneous symbols in virtue of Proposition 7.3.6 and Theorem 7.1.6. Thus we obtain $E_{1} \in I_{\mathrm{hom}}^{-m}\left(X \times X, C^{\prime}\right)$ with $P E_{1}-R \in C^{\infty}$. Since Theorem 4.3.2 shows that $E_{1}$ has the required continuity properties $\mathrm{i}_{\mathrm{t}}$ follows that $E-E_{1}$ satisfies all the conditions (a)-(c). The proof is complete.

\section{References}

(Continued from part I, Acta Math., 127 (1971), 79-183.)

[29]. Andfrsson, K. G., Propagation of analyticity of solutions of partial differential equations with constant coefficients. Ark. Mat., 8 (1970), 277-302.

[30]. Birkhoff, G. D., Dynamical systems. Amer. Math. Soc. Coll. Publ. 9, New York, 1927.

[31]. Bourbaki, N., Espaces vectoriels topologiques. Paris, 1953-55. 
[32]. BJorken, J. D. \& Drell, S., Relativistic quantum fields. Mc Graw-Hill, New York, 1965.

[33]. Courant, R. \& Lax, P. D., The propagation of discontinuities in wave motion. Proc. Nat. Acad. Sci. USA, 42 (1956), 872-876.

[34]. DEWITT, B. S., Dynamical theory of groups and fields. Relativity, groups and topology, 585-820. Gordon and Breach, New York-London, 1964.

[35]. Dieudonne, J. \& Schwartz, L., La dualité dans les espaces $(\mathcal{F})$ et $(\mathcal{L} \mathcal{F})$. Ann. Inst. Fourier (Grenoble), 1 (1949), 61-101.

[36]. Dugundsi, J. \& Antosiewicz, H. A., Parallelizable flows and Lyapunov's second method. Ann. of Math., 73 (1961), 543-555.

[37]. Gårding, L., Kotake, T. \& Leray, J., Uniformisation et développement asymptotique de la solution du problème de Cauchy linéaire, à données holomorphes; analogie avec la théorie des ondes asymptotiques et approchées (Problème de Cauchy Ibis et VI). Bull. Soc. Math. France, 92 (1964), 263-361.

[38]. Grušın, V. V., The extension of smoothness of solutions of differential equations of principal type. Dokl. Akad. Nauk SSSR, 148 (1963), 1241-1244. Also in Soviet Math. Dokl., 4 (1963), 248-252.

[39]. Hadamard, J., Le problème de Cauchy et les équations aux dérivées partielles linéaires hyperboliques. Hermann, Paris 1932.

[40]. Hagflrger, A., Variétés feuilletées. Ann. Scuola Norm. Sup. Pisa, 16 (1962), 367-397.

[4I]. Hörmander, L., Introduction to complex analysis in several variables. D. van Nostrand Publ. Co., Princeton, N. J. 1965.

[42]. - Pseudo-differential operators and non-elliptic boundary problems. Ann. of Math., 83 (1966), 129-209.

[43]. - - On the singularities of solutions of partial differential equations. Comm. Pure Appl. Math., 23 (1970), 329-358.

[44]. - Uniqueness theorems and wave front sets for solutions of linear differential equations with analytic coefficients. Comm. Pure Appl. Math., 24 (1971), 671-704.

[45]. - Linear differential operators. Actes Congr. Intern. Math. Nice, 1970, 1, 121-133.

[46]. - - On the existence and the regularity of solutions of linear pseudo-differential equations. L'Enseignement Math., 17 (1971), 99-163.

[47]. Palats, R., A global formulation of the Lie theory of transformation groups. Mem. Amer. Math. Soc., 22 (1957).

[48]. Malgrange, B., Existence et approximation des solutions des équations aux dérivées partielles et des équations de convolution. Ann. Inst. Fourier (Grenoble), 6 (1955-56), 271-355.

[49]. Riesz, M., L’intégrale de Riemann-Liouville et le problème de Cauchy. Acta Math., 81 (1949), 1-223.

[50]. Stemnrod, N., The topology of fiber bundles. Princeton Univ. Press, Princeton 1951.

[51]. Unterberger, A. \& Bokobza, J., Les opérateurs pseudo-différentiels d'ordre variable. C. R. Acad. Sci. Paris, 261 (1965), 2271-2273.

[52]. Whitney, H., Regular families of curves. Ann. of Math., 34 (1933), 244-270.

[53]. Zarner, M., Solutions singulières d'équations aux dérivées partielles. Bull. Soc. Math. France, 91 (1963), 203-226. 